\title{
Minimizing orbits in the discrete Aubry-Mather model
}

\author{
Eduardo Garibaldi* \\ Departamento de Matemática \\ Universidade Estadual de Campinas \\ 13083-859 Campinas - SP, Brasil \\ garibaldi@ime.unicamp.br
}

\author{
Philippe Thieullen ${ }^{\dagger}$ \\ Institut de Mathématiques \\ Université Bordeaux 1, CNRS, UMR 5251 \\ F-33405 Talence, France \\ Philippe.Thieullen@math.u-bordeaux1.fr
}

July 30, 2010

\begin{abstract}
We consider a generalization of the Frenkel-Kontorova model in higher dimension leading to a new theory of configurations with minimal energy, as in Aubry's theory or in Mather's twist approach in the periodic case. We consider a one dimensional chain of particles and their minimizing configurations and we allow the state of each particle to possess many degrees of freedom. We assume that the Hamiltonian of the system satisfies some twist condition. The usual "total ordering" of minimizing configurations does not exist any more and new tools need to be developed. The main mathematical tool is to cast the study the minimizing configurations into the framework of discrete Lagrangian theory. We introduce forward and backward Lax-Oleinik problems and interpret their solutions as discrete viscosity solutions as in HamiltonJacobi methods. We give a fairly complete description of a particular class of minimizing configurations: the calibrated class. These configurations may be thought of as "ground states" obtained in the thermodynamic limit at temperature zero. We obtain, in particular, Mather's graph property or the non-crossing property of two calibrated configurations and the existence of a rotation number for most of the calibrated configurations.
\end{abstract}

\section{Introduction}

The original Frenkel-Kontorova model, see [29], describes a one dimensional chain of (classical) particles coupled to their nearest neighbors and subjected to a periodic environment generated by a one dimensional cristal. The chain is supposed to be located on a line; the local interaction energy $\mathcal{L}: \mathbb{R}^{2} \rightarrow \mathbb{R}$ takes into account an elastic potential between two successive particles in the chain, as well as an external periodic potential modeling the influence of a periodic structure:

$$
\mathcal{L}\left(x_{k}, x_{k+1}\right)=\frac{1}{2}\left(x_{k+1}-x_{k}-\lambda\right)^{2}+\frac{K}{(2 \pi)^{2}}\left(1-\cos 2 \pi x_{k}\right),
$$

where $\lambda$ is the unperturbed mean interatomic distance and $K$ is the strength of the external periodic environment. The state space in this model is represented by

*supported by CNPq posdoc scholarship

${ }^{\dagger}$ partially supported by ANR BLANC07-3_187245, Hamilton-Jacobi and Weak KAM Theory 
a sequence of positions of each particle constrained to stay on a line. A central problem in this theory is to describe the set of minimizing configurations, that is, configurations which minimize the total energy of any finite sub-chains with fixed boundary states. Among the main results, one may cite the existence of a well defined "rotation number" for any minimizing configuration, the "total ordering property" of the set of minimizing configurations with a fixed rotation number, and the existence of a unique "hull function" up to a phase shift which parametrizes all minimizing configurations with a fixed rotation number. These main results have been obtained by several authors, first by Aubry and Le Daeron [3], Aubry, Axel and Vallet [4], Griffiths, Schellnhuber and Urbschat [37], independently by Mather $[48,51]$, more rencently by Gomes and Oberman, Rorro and Falcone [34, 33, 53, 20], Baesens and MacKay [6], and in a similar context of ergodic optimization by Bousch, Brémont, Jenkinson and Morris, Bressaud and Quas, Leplaideur [9, 10, $12,39,40,13,42]$. The minimizing configurations previously described correspond to equilibrium fixed points of the steady-state Frenkel-Kontorova equation. Recent progress has been made in the space-time Frenkel-Kontorova model. In this model, each particle evolves in time according to a Newtonian second order differential equation. Without being exhaustive, we just quote recent articles by Forcadel, Imbert and Monneau [27, 28] which use viscosity technics related to our methods and by Baesens and MacKay [5].

Several general monographies may help the reading of the present article: for instance, the notes of G. Forni and J. N. Mather [26] for a global description of Aubry-Mather theory, and two textbooks written by G. Contreras and R. Iturriaga [17] and by A. Fathi [23] for the Lagrangian point of view. The weak-KAM approach developed by Fathi for Lagrangian flows is an especially important concept we are going to apply in a discrete setting. For more details on the Frenkel-Kontorova model, we refer the reader to $[11,25]$.

It is crucial to point out that all previously mentioned results (except those in $[34,33])$ depend strongly on the fact that the state $x_{k}$ of the $k^{\text {th }}$ particle of the chain is one dimensional. In other words, the order of the real line plays a crucial role; the fact that two configurations can be compared globally is fundamental. Our focus here is on an extension of Frenkel-Kontorova theory to the multidimensional case: the state of each particle will belong to $\mathbb{R}^{d}$. The case where the state space is finite $\mathcal{S}=\{1,2, \ldots, d\}$ and the interaction energy map $\mathcal{L}(i, j)$ is a function on $\mathcal{S} \times \mathcal{S}$ is a relevant situation worked out in ergodic optimization theory and in Gibbs fields theory. Although we do not consider specific examples nor numerical experiments in this article, the following interaction energy satisfies the hypotheses of all subsequent theorems:

$$
\mathcal{L}\left(x_{k}, x_{k+1}\right)=\frac{1}{2} \sum_{i=1}^{d}\left|x_{k+1}^{i}-x_{k}^{i}-\lambda^{i}\right|^{2}+\frac{K}{(2 \pi)^{2}}\left(1-\cos \left(2 \pi \sum_{i}^{d} \nu^{i} x_{k}^{i}\right)\right),
$$

where $\left(\nu^{1}, \ldots, \nu^{d}\right)$ are integers and $\left(x_{k}^{1}, \ldots, x_{k}^{d}\right)$ are the components of $x_{k}$ in $\mathbb{R}^{d}$.

A large part of the theory of minimizing configurations can be done under very weak assumptions on the local interaction energy $\mathcal{L}(x, y)$. We first assume that 
$\mathcal{L}(x, y): \mathbb{R}^{d} \times \mathbb{R}^{d} \rightarrow \mathbb{R}$ is continuous and is doubly periodic

$$
\mathcal{L}(x, y)=\mathcal{L}(x+s, y+s), \quad \forall s \in \mathbb{Z}^{d} .
$$

We next assume that $\mathcal{L}(x, y)$ is coercive

$$
\lim _{R \rightarrow+\infty} \inf _{\|x-y\| \geq R} \mathcal{L}(x, y)=+\infty .
$$

The formal total energy of a chain of particles $\left\{x_{k}\right\}_{k \in \mathbb{Z}}$ in $\mathbb{R}^{d}$ is given by

$$
\mathcal{L}_{\text {tot }}\left(\left\{x_{k}\right\}_{k \in \mathbb{Z}}\right)=\sum_{k \in \mathbb{Z}} \mathcal{L}\left(x_{k}, x_{k+1}\right),
$$

which may a priori diverge. A minimizing configuration is a configuration that can only increase its total energy whenever a finite number of particles is moved, that is, a configuration $\left\{x_{k}\right\}_{k \in \mathbb{Z}}$ in $\mathbb{R}^{d}$ satisfying

$$
\mathcal{L}\left(x_{m}, x_{m+1}, \ldots, x_{n}\right):=\sum_{k=m}^{n-1} \mathcal{L}\left(x_{k}, x_{k+1}\right) \leq \mathcal{L}\left(y_{m}, y_{m+1}, \ldots, y_{n}\right),
$$

for every $m<n$ and every configuration $\left\{y_{k}\right\}_{k \in \mathbb{Z}}$ in $\mathbb{R}^{d}$ with $y_{m}=x_{m}$ and $y_{n}=x_{n}$. If $\mathcal{L}$ is in addition $C^{1}$, a minimizing configuration $\left\{x_{k}\right\}$ is critical (or at equilibrium) in the sense that:

$$
\frac{\partial \mathcal{L}}{\partial y}\left(x_{k-1}, x_{k}\right)+\frac{\partial \mathcal{L}}{\partial x}\left(x_{k}, x_{k+1}\right)=0, \quad \forall k \in \mathbb{Z} .
$$

The main objective of this paper is to extend partially the theory of FrenkelKontorova model to the case where the state $x_{k}$ of each particle belongs to a $d$ dimensional vector space $\mathbb{R}^{d}$. The natural tool we are going to apply in the multidimensional setting comes from either the notion of effective potential of Chou and Griffiths [15] or the notion of viscosity solution of Fathi's weak KAM theory [23]. This approach allows only the study of a special class of minimizing configurations, called ground states by Chou and Griffiths or calibrated by Fathi. Notice that Aubry-Le Daeron [3] used the expression "ground state" to denote a recurrent minimizing configuration. It happens that, in the one dimensional case, recurrent minimizing configurations and calibrated configurations are strongly related through the notion of rotation number; we do not know whether that fact persists in the multidimensional setting. Ground states are physical quantities as shown by N. Anantharaman in [1]: she proved that Gibbs measures at positive temperatures converge when the system is frozen (in a weak sense along any converging subsequences) to measures whose support contains only ground states. We will show that calibrated configurations exist assuming $\mathcal{L}(x, y)$ to be only $C^{0}$ and coercive. We will also show that most of the calibrated configurations admit a rotation vector assuming in addition $\mathcal{L}(x, y)$ to be superlinear:

$$
\lim _{R \rightarrow+\infty} \inf _{\|y-x\| \geq R} \frac{\mathcal{L}(x, y)}{\|x-y\|}=+\infty .
$$


Uniqueness of a calibrated configuration passing through a given point uses an even stronger assumption called twist condition or ferromagnetic condition (definition 2.4), which is implied, for instance, when $\mathcal{L}(x, y)$ is $C^{2}$ and uniformly strictly convex (definition 2.7). The definition of calibrated configurations will be explained soon.

Generalizations of Frenkel-Kontorova model have been investigated in several different directions. The state space is still one dimensional as in Aubry Mather theory.

One can consider, for example, a multidimensional setting where the particles are indexed by the sites of a multidimensional lattice $\mathbb{Z}^{d}$. The topology of interactions plays a fundamental role. The interaction can be either local with each particle interacting only with its nearest neighbors, or global as in mean field theory. The state $x_{k} \in \mathbb{R}$ of the particles is nevertheless one dimensional as in Aubry theory, but indexed by $\mathbb{Z}^{d}$ for instance. Notions of minimizing configurations and rotation vectors can be defined similarly. In the context of the multidimensional Frenkel-Kontorova model, one still obtains minimizing configurations having a prescribed rotation vector. For precise definitions and statements, we refer the reader to the work of R. de la Llave and E. Valdinoci [43]. One should also consult the paper of H. Koch, R. de la Llave and C. Radin [41] for situations where the variables range over a more complicated lattice.

In another direction, the potential could be assumed quasiperiodic instead of periodic, as it is done in the work of J. M. Gambaudo, P. Guiraud and S. Petite [30]. Once again, the state space is one dimensional, all minimizing configurations admit a rotation number and all rotation number is obtained by a minimizing configuration.

Mather [51] has developed a theory in any dimension, closely related to the present article, of minimizing measures for a Tonelli's Lagrangian, that is, 1-periodic superlinear strictly convex Lagrangian $L: T M \times \mathbb{T}^{1} \rightarrow \mathbb{R}$. In dimension 1 , Moser [52] has proved the equivalence between two approaches, monotone twist map versus Tonelli's Lagrangian, showing that such a monotone twist map can always be seen as the time one map of some 1-periodic smooth Hamiltonian $H: T^{*} M \times \mathbb{T}^{1} \rightarrow \mathbb{R}$. As noticed by Herman [38], there are interesting connections between configurations with minimal energy and Lagrangian tori invariant under symplectic diffeormorphisms of the cotangent bundle of the $d$-dimensional torus. Massart has generalized several results of the Aubry-Mather theory in [45, 46, 47].

We now return to the main results of the present article. We first define the ground energy per particle or the minimizing holonomic value $\overline{\mathcal{L}}$. The word holonomic is used as in Gomes [34] or Mañé [44] to point out that no dynamical system can be introduced in a natural way. More explanations on this subject will be given in section 3. The minimizing holonomic value may be seen as the lowest mean energy per particle, formally it is defined as

$$
\overline{\mathcal{L}}=\inf \left\{\liminf _{n \rightarrow+\infty} \frac{1}{n} \mathcal{L}\left(x_{0}, x_{1}, \ldots, x_{n}\right):\left\{x_{k}\right\}_{k \in \mathbb{Z}} \text { is a configuration }\right\} .
$$

We will describe a special subset of minimizing configurations called calibrated, which is closely related to Aubry's notion of recurrent minimizing configuration or 
to Chou and Griffiths' concept of ground state. We first define the Aubry set $\mathcal{A}(\mathcal{L})$, a similar notion introduced by Fathi [23]. Informally, $\mathcal{A}(\mathcal{L})$ is the set of configurations $\left\{x_{k}\right\}_{k \in \mathbb{Z}}$ such that all couple $\left(x_{k}, x_{k+1}\right)$ is the initial segment of a periodic configurations with minimal energy. More precisely, $\left\{x_{k}\right\}_{k \in \mathbb{Z}} \in \mathcal{A}(\mathcal{L})$ if, and only if, for every $k \in \mathbb{Z}$ and $\epsilon>0$, there exist a periodic configuration $\left(x_{0}^{\epsilon}, x_{1}^{\epsilon}, \ldots, x_{q(\epsilon)}^{\epsilon}\right)$ and integers $q(\epsilon) \geq 1$ and $p(\epsilon)$ such that $x_{q(\epsilon)}^{\epsilon}=x_{0}^{\epsilon}+p(\epsilon)$,

$$
\left|\mathcal{L}\left(x_{0}^{\epsilon}, x_{1}^{\epsilon}, \ldots, x_{q(\epsilon)}^{\epsilon}\right)-q(\epsilon) \overline{\mathcal{L}}\right|<\epsilon \quad \text { and } \quad\left(x_{k}, x_{k+1}\right)=\left(x_{0}^{\epsilon}, x_{1}^{\epsilon}\right) .
$$

It is not difficult but not immediate to show that configurations in the Aubry set are minimizing in a stronger sense. More precisely, they are minimizing with respect to $\mathcal{L}^{*}(x, y):=\inf _{s, t \in \mathbb{Z}^{d}} \mathcal{L}(x+s, y+t)$. Notice that the two minimizing holonomic values are identical: $\mathcal{L}=\overline{\mathcal{L}}^{*}$. Let $\mathcal{E}(\mathcal{L})$ be the set of energy minimizing configurations in the strong sense. Configurations in $\mathcal{E}(\mathcal{L})$ are strongly related to a special class of configurations called calibrated.

We first introduce an important notion called effectif potential by Chou and Griffiths, weak KAM solution by Fathi or viscosity solution in the context of Hamilton-Jacobi equation. We prefer to use the term calibrated sub-action. We call sub-action any continuous $\mathbb{Z}^{d}$-periodic function $u: \mathbb{R}^{d} \rightarrow \mathbb{R}$ such that

$$
u(y)-u(x) \leq \mathcal{L}(x, y)-\overline{\mathcal{L}}, \quad \forall x, y \in \mathbb{R}^{d} .
$$

We call backward or forward calibrated sub-action a sub-action $u$ satisfying in addition

$$
\left\{\begin{array}{cll}
\text { the forward case: } & u(x)-\overline{\mathcal{L}}=\max _{y \in \mathbb{R}^{d}}[u(y)-\mathcal{L}(x, y)], & \forall x \in \mathbb{R}^{d}, \\
\text { the backward case: } & u(y)+\overline{\mathcal{L}}=\min _{x \in \mathbb{R}^{d}}[u(x)+\mathcal{L}(x, y)], & \forall y \in \mathbb{R}^{d} .
\end{array}\right.
$$

Calibrated sub-actions $u$ are solutions of the backward or forward Lax-Oleinik operator. These operators may be seen as a min-plus (or tropical) version of the Ruelle operator used in the thermodynamical formalism. The two sub-actions may be seen as left and right eigenvectors of a min-plus eigenvalue problem. We not only guarantee that calibrated sub-actions do exist in the $C^{0}$ coercive case, but we also discuss how the regularity of the local interaction energy affects the regularity of a calibrated sub-action. We show (see proposition 4.7) that, if $\mathcal{L}$ is locally Lipschitz or $C^{2}$, then any forward (resp. backward) calibrated sub-action is Lipschitz or semiconvex (resp. semiconcave). See also Gomes [34] for similar results.

We call calibrated configuration (or more precisely $u$-calibrated if needed) a configuration $\left\{x_{k}\right\}_{k \in \mathbb{Z}}$ such that, for some sub-action $u$,

$$
u\left(x_{k+1}\right)-u\left(x_{k}\right)=\mathcal{L}\left(x_{k}, x_{k+1}\right)-\overline{\mathcal{L}}, \quad \forall k \in \mathbb{Z} .
$$

Let $\mathcal{N}(\mathcal{L}, u)$ be the set of $u$-calibrated configurations. It is then obvious that a calibrated configuration is minimizing in the strong sense. It is also easy to see that configurations in the Aubry set are calibrated for any sub-action and therefore minimizing in the strong sense:

$$
\mathcal{A}(\mathcal{L}) \subset \mathcal{N}(\mathcal{L}, u) \subset \mathcal{E}(\mathcal{L}), \quad \forall u \text { calibrated }
$$


We show in section 5 that calibrated configurations do exist and have bounded jumps $\sup _{k}\left\|x_{k+1}-x_{k}\right\|<+\infty$. We also show in section 10 that the Aubry set can be characterized using the property of calibrated sub-actions, more precisely

$$
\mathcal{A}(\mathcal{L})=\left\{\underline{x}=\left\{x_{k}\right\}_{k \in \mathbb{Z}} \in\left(\mathbb{R}^{d}\right)^{\mathbb{Z}}: \underline{x} \text { is calibrated for any sub-action }\right\} .
$$

The proof requires a particular class of sub-actions, called separating sub-action. The fact that the class of separating sub-actions is generic in the $C^{0}$ topology may be seen as a discrete counterpart of Fathi and Siconolfi's critical subsolutions in the framework of Hamilton-Jacobi equation (see [24]).

In the $C^{1}$ ferromagnetic and coercive case, we can say more on the Aubry set. For any $x_{0} \in \mathbb{R}^{d}$, there exists at most one calibrated configuration passing through $x_{0}$. The two nearest neighbors are given by the equation

$$
D u\left(x_{0}\right)=\frac{\partial \mathcal{L}}{\partial y}\left(x_{-1}, x_{0}\right)=-\frac{\partial \mathcal{L}}{\partial x}\left(x_{0}, x_{1}\right),
$$

where $u$ is any sub-action and $D u$ is continuous on the projected Aubry set. This property is called the Aubry-Mather graph property. The graph is compact and invariant with respect to the corresponding twist or discrete Euler-Lagange map.

Sections 6 and 7 are devoted to more precise properties of the Aubry set in the general $C^{0}$ coercive case. We show that the Aubry set is not empty by showing that it contains the Mather set $\mathcal{M}(\mathcal{L})$ which is not empty. The Mather set is built using minimizing holonomic measures, a notion introduced by Mather (see [49] and [51]) but in the context of twist maps where the invariance property is used instead of the holonomic property. In the context of configurations space, we call minimizing holonomic measure a transshipment $\pi(d x, d y)$ (definition 3.3), that is, a sigmafinite measure $\pi(d x, d y)$ invariant by the integer translations $x \in \mathbb{R}^{d} \mapsto x+k \in \mathbb{R}^{d}$, $k \in \mathbb{Z}^{d}$, finite with mass one on any fundamental domain, say $\mathbb{T}^{d} \times \mathbb{R}^{d}$, with identical marginals on the two factors $\mathbb{R}^{d}, p r_{*}^{1}(\pi)=p r_{*}^{2}(\pi)$, which in addition minimizes

$$
\pi=\operatorname{argmin} \iint_{\mathbb{T}^{d} \times \mathbb{R}^{d}} \mathcal{L}(x, y) \pi(d x, d y) .
$$

We show that the minimum is attained by such transshipments with bounded offdiagonal support. We call Mather set $\mathcal{M}(\mathcal{L})$ the set of configurations $\left\{x_{k}\right\}_{k \in \mathbb{Z}}$ such that, for all $k \in \mathbb{Z}$, there exists a minimizing holonomic measure $\pi$ whose support contains $\left(x_{k}, x_{k+1}\right)$. We then show that

$$
\mathcal{M}(\mathcal{L}) \subset \mathcal{A}(\mathcal{L}) \quad \text { and } \quad \overline{\mathcal{L}}=\iint_{\mathbb{T}^{d} \times \mathbb{R}^{d}} \mathcal{L}(x, y) \pi(d x, d y), \forall \pi \text { minimizing. }
$$

The Aubry set may contain "heteroclinic" configurations joining two minimizing periodic orbits and may therefore be stricly larger than the Mather set. Aubry [3] uses the term advanced or delayed discommensuration for these minimizing configurations. The Aubry set admits a description in terms of a potential barrier. We call Mañé potential the map

$$
S(x, y)=\inf \left\{\mathcal{L}\left(x_{0}, \ldots, x_{n}\right)-n \overline{\mathcal{L}}: n \geq 1, x_{0}=x, x_{n}=y+p, x_{k} \in \mathbb{R}^{d}, p \in \mathbb{Z}^{d}\right\} .
$$


Chou and Griffiths call excitation energy of a configuration $\left\{x_{k}\right\}_{k \in \mathbb{Z}}$, relative to the ground state level, the nonnegative limit

$$
\lim _{k \rightarrow-\infty, l \rightarrow+\infty}\left[\mathcal{L}\left(x_{k}, x_{k+1}, \ldots, x_{l}\right)-(l-k) \overline{\mathcal{L}}\right]-S\left(x_{k}, x_{l}\right) .
$$

A calibrated configuration has obviously zero excitation energy. We show that the Aubry set can be characterized by the Mañé potential and coincides with the set of pure ground states introduced by Chou and Griffiths, namely,

$$
\begin{aligned}
\mathcal{A}(\mathcal{L}) & =\left\{\left\{x_{k}\right\}_{k \in \mathbb{Z}}: \mathcal{L}\left(x_{k}, x_{k+1}\right)-\overline{\mathcal{L}}=S\left(x_{k}, x_{k+1}\right)=-S\left(x_{k+1}, x_{k}\right), \forall k \in \mathbb{Z}\right\} \\
& =\left\{\left\{x_{k}\right\}_{k \in \mathbb{Z}}: \mathcal{L}\left(x_{k}, \ldots, x_{l}\right)-(l-k) \overline{\mathcal{L}}=S\left(x_{k}, x_{l}\right)=-S\left(x_{l}, x_{k}\right), \forall k<l\right\} .
\end{aligned}
$$

Assuming only $\mathcal{L}$ to be $C^{0}$ and coercive, we prove in section 8 that $S(x, y)$ is continuous, doubly periodic and satisfies $u(y)-u(x) \leq S(x, y)$, for all $x, y \in \mathbb{R}^{d}$ and any sub-action $u$. Let $\mathcal{A}^{0}(\mathcal{L})$ be the projected Aubry set,

$$
\mathcal{A}^{0}(\mathcal{L})=\left\{x_{0} \in \mathbb{R}^{d}:\left\{x_{k}\right\}_{k \in \mathbb{Z}} \in \mathcal{A}(\mathcal{L})\right\} .
$$

We show that (see theorem 8.10), for any $x \in \mathcal{A}^{0}(\mathcal{L}), S(x, \cdot)$ is backward calibrated, $-S(\cdot, x)$ is forward calibrated and a characterization in terms of Mañé potential:

$$
\mathcal{A}^{0}(\mathcal{L})=\left\{x_{0} \in \mathbb{R}^{d}: S\left(x_{0}, x_{0}\right)=0\right\} .
$$

We finally show (see theorem 9.1) that calibrated sub-actions are completely known as soon as they are known on the projected Aubry set:

$$
\left\{\begin{array}{cll}
\text { the forward case: } & u(x)=\max _{y \in \mathcal{A}^{0}(\mathcal{L})}[u(y)-S(x, y)], & \forall x \in \mathbb{R}^{d}, \\
\text { the backward case: } & u(y)=\min _{x \in \mathcal{A}^{0}(\mathcal{L})}[u(x)+S(x, y)], & \forall y \in \mathbb{R}^{d} .
\end{array}\right.
$$

This result should be compared to the one obtained by G. Contreras [16] for weak KAM solutions.

When a system depends on several parameters, it is important to introduce an order parameter which labels the different phases in a bifurcation diagram called phase diagram. In the one dimensional case, the order parameter is the rotation number of ground states. It is generally believed that, in the $(\lambda, K)$ phase diagram in the standard Frenkel-Kontorova, apart a set of zero Lebesgue measure, the Aubry set is reduced to a unique periodic orbit: the system is locked at rational rotation number. We introduce in section 11 the basis of a theory of rotation vectors in the multidimensional case.

We call rotation vector $\omega$ of a minimizing configuration $\left\{x_{k}\right\}_{k \in \mathbb{Z}}$ the following limit when it exists

$$
\omega\left[\left\{x_{k}\right\}_{k \in \mathbb{Z}}\right]=\lim _{n-m \rightarrow+\infty} \frac{x_{n}-x_{m}}{n-m} .
$$

As in Mather theory, we relate this notion to the notion of rotation vector of a minimizing measure

$$
\omega[\pi]=\iint_{\mathbb{T}^{d} \times \mathbb{R}^{d}}(y-x) \pi(d x, d y) .
$$

The main important observation is that a configuration is minimizing for the interaction energy $\mathcal{L}(x, y)$ if, and only if, it is minimizing for the interaction energy 
$\mathcal{L}(x, y)-\langle\lambda,(y-x)\rangle$ for any parameter $\lambda \in \mathbb{R}^{d}$. In the one dimensional case, a recurrent minimizing configuration admits a rotation number $\omega$ and is calibrated for some $\mathcal{L}-\lambda$. The two numbers $\omega$ and $\lambda$ are related by Legendre transform. If $\omega$ is irrational, $\lambda$ is unique. In the multidimensional case, we follow Gomes [34] to show that, whenever the minimizing holonomic value of $\mathcal{L}-\lambda$, denoted $\overline{\mathcal{L}}(\lambda)$, is differentiable at $\lambda$, any calibrated configuration for $\mathcal{L}-\lambda$ admits a rotation vector given by

$$
\omega=-\frac{\partial \overline{\mathcal{L}}}{\partial \lambda}
$$

In particular, there exists minimizing configuration with rotation vector of arbitrarily large norm.

We have chosen to translate the Frenkel-Kontorova model into the framework of discrete Lagrangian Aubry-Mather theory mainly to have a simple notion of minimizing holonomic measures instead of transshipments. The main object we are interested in is thus a $C^{0}$ coercive Lagrangian $L(x, v)$ defined on $\mathbb{T}^{d} \times \mathbb{R}^{d}$. A family of local interaction energies can be naturally defined by

$$
\mathcal{L}_{\tau}(x, y)=\tau L\left(x\left(\bmod \mathbb{Z}^{d}\right), \frac{y-x}{\tau}\right), \quad \forall x, y \in \mathbb{R}^{d},
$$

where $\tau>0$ is a parameter that we keep in order to understand later the homogenization theory of Frenkel-Kontorova model as in [27] and [28]. Notice that $\mathcal{L}_{\tau}$ is invariant under the diagonal action of $\mathbb{Z}^{d}$,

$$
\mathcal{L}_{\tau}(x+k, y+k)=\mathcal{L}_{\tau}(x, y), \quad \forall k \in \mathbb{Z}^{d} .
$$

The two approches are complementary. While the Lagrangian formulation will be more adapted in the description of the support of minimizing measures, the FrenkelKontorova setting will be used in the construction of calibrated sub-actions, as well as in the definition of two major notions of action potential: the Mañé potential and the Peierls barrier. We intend later to better understand the limit when the step $\tau$ tends to zero and the thermodynamic ground state limit of the system when the temperature goes to zero.

\section{$2 \quad$ A discrete Lagrangian dynamics}

We fix from now on a $C^{0}$ coercive Lagrangian $L(x, v): \mathbb{R}^{d} \times \mathbb{R}^{d} \rightarrow \mathbb{R}, \mathbb{Z}^{d}$-periodic in $x$, and its associated local interaction energy map

$$
\mathcal{L}_{\tau}(x, y)=\tau L\left(x, \frac{y-x}{\tau}\right)
$$

defined on $\mathbb{R}^{d} \times \mathbb{R}^{d}$ and invariant by the diagonal action of $\mathbb{Z}^{d}$. We begin by recalling some well known notions of divergence type at infinity. Coerciveness is our basic assumption, superlinerarity will be used when homology will play a role.

Definition 2.1. Let $L(x, v): \mathbb{T}^{d} \times \mathbb{R}^{d} \rightarrow \mathbb{R}$ be a $C^{0}$-Lagrangian .

i. $L(x, v)$ is said to be coercive if $\lim _{R \rightarrow+\infty} \inf _{\|v\| \geq R} \inf _{x \in \mathbb{T}^{d}} L(x, v)=+\infty$. 
ii. $L(x, v)$ is said to be superlinear if $\lim _{R \rightarrow+\infty} \inf _{\|v\| \geq R} \inf _{x \in \mathbb{T}^{d}} \frac{L(x, v)}{\|v\|}=+\infty$.

We call configuration any sequence $\left\{x_{k}\right\}_{k \in \mathbb{Z}}$ of points in $\mathbb{R}^{d}$. Let $\Sigma=\left(\mathbb{R}^{d}\right)^{\mathbb{Z}}$ be the set of configurations. We also consider the set of configurations modulo the diagonal action of $\mathbb{Z}^{d}$, that is, the quotient of $\Sigma$ by the equivalence relation: $\left\{x_{k}\right\}_{k \in \mathbb{Z}} \sim\left\{y_{k}\right\}_{k \in \mathbb{Z}}$ if, and only if, there exists $s \in \mathbb{Z}^{d}$ such that $y_{k}=x_{k}+s$ for all $k \in \mathbb{Z}$. So

$$
\Sigma=\left(\mathbb{R}^{d}\right)^{\mathbb{Z}} \text { and } \Sigma / \sim=\left(\mathbb{R}^{d}\right)^{\mathbb{Z}} / \sim .
$$

Let us notice that, for any fundamental domain $\mathcal{D}$ of the action of $\mathbb{Z}^{d}$ on $\mathbb{R}^{d}$, the set $\left(\mathbb{R}^{d}\right)^{\mathbb{Z}_{-}^{*}} \times \mathcal{D} \times\left(\mathbb{R}^{d}\right)^{\mathbb{Z}_{+}^{*}}$ is a fundamental domain for the diagonal action of $\mathbb{Z}^{d}$ on $\Sigma$. Let $\sigma: \Sigma \rightarrow \Sigma$ be the left shift given by $\sigma\left(\left\{x_{k}\right\}\right)=\left\{y_{k}\right\}$ where $y_{k}=x_{k+1}$. Notice that $\sigma$ commutes with the diagonal action.

Definition 2.2. We call minimizing configuration any sequence $\left\{x_{k}\right\}_{k \in \mathbb{Z}}$ which minimizes the local interaction energy, namely,

$$
\mathcal{L}_{\tau}\left(x_{n}, x_{n+1}, \ldots, x_{n+m}\right):=\sum_{k=n}^{n+m} \mathcal{L}_{\tau}\left(x_{k}, x_{k+1}\right) \leq \mathcal{L}_{\tau}\left(y_{n}, y_{n+1}, \ldots, y_{n+m}\right),
$$

for any finite configuration $\left\{y_{k}\right\}_{k=n}^{n+m}$ with identical boundary conditions $x_{n}=y_{n}$ and $x_{n+m}=y_{n+m}$.

Although one of our aim is to extend as much as we can the discrete AubryMather theory to just $C^{0}$ coercive Lagrangian and to describe precisely the set of minimizing configurations in this general setting, we show in this section that, under a stronger hypothesis on the Lagrangian $\left(C^{2}\right.$-smoothness and twist condition), we can recover the original theory, where the set of minimizing configurations can be understood through the help of a dynamical system similar to the usual standard map. Let us first recall the notion of critical configuration.

Definition 2.3. Let $L(x, v): \mathbb{T}^{d} \times \mathbb{R}^{d} \rightarrow \mathbb{R}$ be a $C^{1}$-Lagrangian. We call critical triple a configuration $\left(x_{-1}, x_{0}, x_{1}\right)$ of three points in $\mathbb{R}^{d}$ satisfying

$$
\frac{\partial \mathcal{L}_{\tau}}{\partial y}\left(x_{-1}, x_{0}\right)+\frac{\partial \mathcal{L}_{\tau}}{\partial x}\left(x_{0}, x_{1}\right)=0
$$

We call critical configuration any configuration $\left\{x_{k}\right\}_{k \in \mathbb{Z}}$ of points in $\mathbb{R}^{d}$ consisting of critical triples $\left(x_{k-1}, x_{k}, x_{k+1}\right)$ :

$$
\frac{\partial \mathcal{L}_{\tau}}{\partial y}\left(x_{k-1}, x_{k}\right)+\frac{\partial \mathcal{L}_{\tau}}{\partial x}\left(x_{k}, x_{k+1}\right)=0, \quad \forall k \in \mathbb{Z}
$$

Let $\Gamma_{\tau}(L) \subset \Sigma$ be the set of critical configurations. We notice that $\Gamma_{\tau}(L)$ is invariant by both the diagonal action of $\mathbb{Z}^{d}$ and the shift $\sigma$. Let $\Gamma_{\tau}(L) / \sim$ be the quotient of $\Gamma_{\tau}(L)$ by the diagonal action of $\mathbb{Z}^{d}$. 
The equations defining $\Gamma_{\tau}(L)$ may be seen as a discrete version of the EulerLagrange equation. These equations show that, under some stronger hypothesis of twist condition, the knowledge of $\left(x_{0}, x_{1}\right)$ implies the existence of a unique critical configuration with such initial conditions. More precisely, prefering the use of the ferromagnetic terminology instead of the twist condition as it is done in statistical mechanics, we introduce the following notion.

Definition 2.4. A $C^{1}$-Lagrangian $L(x, v)$ is said to be ferromagnetic if, for any sufficiently small $\tau>0$, the two maps in (I) or equivalently in (II), where

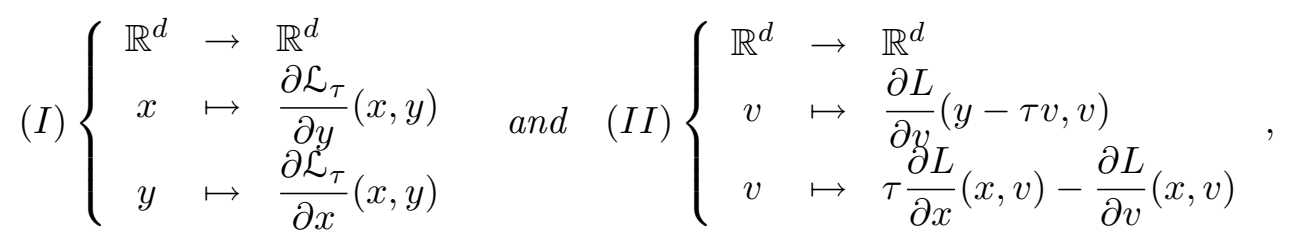

are homeomorphisms for all $(x, y)$.

Similarly a discrete version of the Euler-Lagrange flow may be introduced.

Definition 2.5. Let $L(x, v)$ be a $C^{1}$ ferromagnetic Lagrangian. For sufficiently small $\tau>0$, we call discrete Euler-Lagrange map (or standard map), the map

$$
\Phi_{\tau}=\left\{\begin{array}{ccc}
\mathbb{T}^{d} \times \mathbb{R}^{d} & \rightarrow \mathbb{T}^{d} \times \mathbb{R}^{d} \\
(x, v) & \mapsto(y, w)
\end{array}\right.
$$

where $y=x+\tau v$ and $w$ is the unique solution of one of the two equivalent equations

$$
\frac{\partial \mathcal{L}_{\tau}}{\partial y}(x, y)+\frac{\partial \mathcal{L}_{\tau}}{\partial x}(y, y+\tau w)=0 \quad \text { or } \quad \frac{\partial L}{\partial v}(x, v)+\tau \frac{\partial L}{\partial x}(y, w)-\frac{\partial L}{\partial v}(y, w)=0 .
$$

Notice that $\Phi_{\tau}$ is a homeomorphism on $\mathbb{T}^{d} \times \mathbb{R}^{d}$. In most part of the article, the dynamical sytem $\left(\mathbb{T}^{d} \times \mathbb{R}^{d}, \Phi_{\tau}\right)$ will not be used, except, for instance, in section 6 , where we prove that minimizing measures are supported on a graph. The main advantage of the standard map approach is that the space of critical configurations modulo the diagonal action is conjugate to a $2 d$ degrees of freedom dynamical system.

Remark 2.6. Let $\Pi_{\tau}: \mathbb{R}^{d} \times \mathbb{R}^{d} \rightarrow \mathbb{T}^{d} \times \mathbb{R}^{d}$ be the projection given by

$$
\Pi_{\tau}\left(x_{0}, x_{1}\right)=\left(x_{0} \bmod \mathbb{Z}^{d},\left(x_{1}-x_{0}\right) / \tau\right) .
$$

We extend $\Pi_{\tau}$ to $\Sigma$ by writing $\Pi_{\tau}\left(\left\{x_{k}\right\}_{k \in \mathbb{Z}}\right)=\Pi_{\tau}\left(x_{0}, x_{1}\right)$ and notice that the projection $\Pi_{\tau}: \Sigma / \sim \rightarrow \mathbb{T}^{d} \times \mathbb{R}^{d}$ is also well defined. If $L(x, v)$ is ferromagnetic, then $\left(\Gamma_{\tau}(L) / \sim, \sigma\right)$ is conjugated to $\left(\mathbb{T}^{d} \times \mathbb{R}^{d}, \Phi_{\tau}\right)$, that is, the following diagram commutes

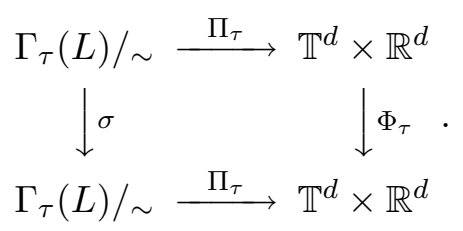


A critical configuration is thus completely determined by the $2 d$ data $(x, v)$ and a general configuration plays the role of a virtual deformation as in Mechanics. In order to check that a Lagrangian satisfies the ferromagnetic condition, an easier but stronger assumption may be used instead.

Definition 2.7. Let $L(x, v): \mathbb{T}^{d} \times \mathbb{R}^{d} \rightarrow \mathbb{R}$ be a $C^{2}$-Lagrangian. We say that $L$ is uniformly strictly convex (with respect to $v$ ) if $\frac{\partial^{2} L}{\partial v^{2}}$ is uniformly positive definite, that is, if there exists $\alpha>0$ such that

$$
\left\langle\frac{\partial^{2} L}{\partial v^{2}}(x, v) \cdot w, w\right\rangle \geq \alpha\|w\|^{2}, \quad \forall x \in \mathbb{T}^{d}, \quad \forall v, w \in \mathbb{R}^{d} .
$$

The following proposition shows that a strictly convex Lagrangian with bounded second derivative is ferromagnetic. The proof is left to the reader.

Proposition 2.8. Let $L(x, v): \mathbb{T}^{d} \times \mathbb{R}^{d} \rightarrow \mathbb{R}$ be a $C^{2}$ strictly convex Lagrangian. Then $L(x, v)$ is superlinear. If $L(x, v)$ satisfies in addition the uniform condition $\left\|\frac{\partial^{2} L}{\partial x \partial v}\right\|_{\mathbb{T}^{d} \times \mathbb{R}^{d}} \leq \beta$ for some $\beta>0$, then $L(x, v)$ is ferromagnetic. Moreover, for any $x, y \in \mathbb{T}^{d}$, for any sufficiently small $\tau>0$, the two maps

$$
v \in \mathbb{R}^{d} \mapsto \tau \frac{\partial L}{\partial x}(x, v)-\frac{\partial L}{\partial v}(x, v) \in \mathbb{R}^{d} \quad \text { and } \quad v \in \mathbb{R}^{d} \mapsto \frac{\partial L}{\partial v}(y-\tau v, v) \in \mathbb{R}^{d},
$$

or equivalently the two maps

$$
y \in \mathbb{R}^{d} \mapsto \frac{\partial \mathcal{L}_{\tau}}{\partial x}(x, y) \in \mathbb{R}^{d} \quad \text { and } \quad x \in \mathbb{R}^{d} \mapsto \frac{\partial \mathcal{L}_{\tau}}{\partial y}(x, y) \in \mathbb{R}^{d},
$$

are $C^{1}$-diffeomorphisms. In particular, the discrete Euler-Lagrange map $\Phi_{\tau}$ is a $C^{1}$-diffeomorphism.

Notice that the ferromagnetic condition can be obtained under weaker hypotheses. For instance, assume $d=1$. Let $f: \mathbb{R} \rightarrow \mathbb{R}$ be an increasing homemorphism with $f(0)=0$ and $g: \mathbb{T}^{1} \rightarrow \mathbb{R}$ be a $C^{1}$-function. Then $L(x, v)=g(x)+\int_{0}^{v} f(w) d w$ is superlinear, ferromagnetic and $C^{1}$. We also notice that $\frac{\partial L}{\partial x}$ is a coboundary under the dynamics $\left(\mathbb{T}^{d} \times \mathbb{R}^{d}, \Phi_{\tau}\right)$ :

$$
\tau \frac{\partial L}{\partial x}(y, w)=\frac{\partial L}{\partial v}(y, w)-\frac{\partial L}{\partial v} \circ \Phi_{\tau}^{-1}(y, w) .
$$

\section{Minimizing holonomic measures}

In this section, we would like to make clear the usefulness of the notions of holonomic measure (definition 3.1) and minimizing holonomic measure (definition 3.5). We begin by briefly recalling Mather's approach for the theory of minimizing orbits: $L(x, v)$ is usually assumed to be $C^{2}$, periodic in $x$ (namely, $x \in \mathbb{T}^{d}$ ), strictly convex in $v \in \mathbb{R}^{d}$ and, for the purposes of this article, time independent.

In Mather's approach, we are interested in finding minimizing absolutely continuous trajectories, that is, trajectories $t \in \mathbb{R} \mapsto x(t)$ such that, for any $t_{0}<t_{1}$ and any other trajectory $t \in\left[t_{0}, t_{1}\right] \mapsto y(t)$ satisfying the boundary conditions 
$x\left(t_{0}\right)=y\left(t_{0}\right)$ and $x\left(t_{1}\right)=y\left(t_{1}\right)$, the local action of $x(t)$ on $\left[t_{0}, t_{1}\right]$ is bounded from above by the local action of $y(t)$,

$$
\int_{t_{0}}^{t_{1}} L(x(t), \dot{x}(t)) d t \leq \int_{t_{0}}^{t_{1}} L(y(t), \dot{y}(t)) d t .
$$

These mimimizing trajectories may be looked for among the trajectories with a prescribed rotation vector $\omega \in \mathbb{R}^{d}$,

$$
\lim _{t \rightarrow+\infty} \frac{x(t)}{t}=\lim _{t \rightarrow+\infty} \frac{1}{t} \int_{0}^{t} \dot{x}(t) d t=\omega .
$$

Notice that a minimizing trajectory must satisfy the Euler-Lagrange equation

$$
\frac{d}{d t}\left(\frac{\partial L}{\partial v}(x, \dot{x})\right)=\frac{\partial L}{\partial x}(x, \dot{x})
$$

and is therefore governed by the Euler-Lagrange flow $\Phi_{\tau}\left(x_{0}, \dot{x}_{0}\right)=\left(x_{t}, \dot{x}_{t}\right)$. Suppose in addition that $\left(x_{t}, \dot{x}_{t}\right)$ is recurrent or more precisely is regular in the sense of Birkhoff's ergodic theorem for some $\Phi_{\tau}$-invariant ergodic probability measure $\mu$ on $\mathbb{T}^{d} \times \mathbb{R}^{d}$, then

$$
\iint_{\mathbb{T}^{d} \times \mathbb{R}^{d}} L(x, v) d \mu(x, v) \leq \iint_{\mathbb{T}^{d} \times \mathbb{R}^{d}} L(x, v) d \nu(x, v)
$$

for any other invariant probability measure $\nu$ on $\mathbb{T}^{d} \times \mathbb{R}^{d}$.

It is therefore natural to look for minimizing trajectories as regular orbits of the Euler-Lagrange flow located in the support of minimizing measures. Mather's approach can thus be translated into a linear optimization problem

$$
\left\{\begin{array}{l}
\mu=\operatorname{argmin} \iint_{\mathbb{T}^{d} \times \mathbb{R}^{d}} L(x, v) d \mu(x, v) \\
\mu \text { is a } \Phi_{\tau^{-i n v a r i a n t ~ p r o b a b i l i t y ~ m e a s u r e ~}} \\
\iint_{\mathbb{T}^{d} \times \mathbb{R}^{d}} v d \mu(x, v)=\omega .
\end{array}\right.
$$

Following R. Mañé [44] and D. A. Gomes [34], one can weaken this optimization problem by asking $\mu$ to be only holonomic, that is, satisfying

$$
\int_{\mathbb{T}^{d}} \phi \circ \Phi_{\tau}(x, v) d \mu(x, v)=\int_{\mathbb{T}^{d}} \phi(x) d \mu(x, v)
$$

for any bounded Borel (periodic) function $\phi: \mathbb{T}^{d} \rightarrow \mathbb{R}$. (Notice that the previous condition does not tell that $\mu$ is invariant by $\Phi_{\tau}$ since the the test function $f$ depends only on $x$ and not on $(x, v)$, see definition 3.1).

We come back to our discrete Aubry-Mather theory and, as in the weak Mather's approach, we try to look for minimizing configurations located in the support of minimizing invariant measures or more precisely in the support of minimizing holonomic measures since the discrete Euler-Lagrange map may not exist. We denote by $\mathcal{P}\left(\mathbb{T}^{d} \times \mathbb{R}^{d}\right)$ the convex set of probability measures over the Borel sets of $\mathbb{T}^{d} \times \mathbb{R}^{d}$. 
Definition 3.1. We call holonomic measure a probability measure $\mu \in \mathcal{P}\left(\mathbb{T}^{d} \times \mathbb{R}^{d}\right)$ satisfying

$$
\iint_{\mathbb{T}^{d} \times \mathbb{R}^{d}} \phi(x+\tau v) d \mu(x, v)=\iint_{\mathbb{T}^{d} \times \mathbb{R}^{d}} \phi(x) d \mu(x, v)
$$

for any bounded Borel function $\phi: \mathbb{T}^{d} \rightarrow \mathbb{R}$. The set of holonomic measures is denoted by $\mathcal{P}_{\tau}\left(\mathbb{T}^{d} \times \mathbb{R}^{d}\right)$.

Notice that, in the ferromagnetic case, $\Phi_{\tau}$-invariant probability measures are holonomic. Nevertheless, the holonomic class is larger. For example, any finite configuration $\left(x_{0}, x_{1}, \ldots, x_{n-1}\right)$ gives a holonomic probability $\mu=\frac{1}{n} \sum_{i=0}^{n-1} \delta_{\left(x_{i}, v_{i}\right)}$, where $v_{i}=\frac{x_{i+1}-x_{i}}{\tau}$ and $x_{n}=x_{0}$. Notice also that the set of holonomic measures is closed under the narrow topology.

We want to show that, although the notion of holonomic measures seems to be unrelated to a dynamical system, the set of these measures is nevertheless in one-to-one correspondence with the set of normalized invariant Markov chain of $(\Sigma, \sigma)$.

Definition 3.2. We call normalized invariant Markov chain on $(\Sigma, \sigma)$ a sigmafinite Markov chain $(\nu(d x), p(x, d y)$ ), with initial distribution $\nu(d x)$ (a sigma-finite measure defined on the Borel sets of $\mathbb{R}^{d}$ ) and transition kernel $p(x, d y)$ (a measurable family of probability measures defined on the Borel sets of $\mathbb{R}^{d}$ ), satisfying the following properties:

i. $\nu(d x)$ is invariant under the action of $\mathbb{Z}^{d}$ and has mass one on any fundamental domain,

ii. $p(x, d y)$ is invariant under the action of $\mathbb{Z}^{d}$ in the following sense

$$
\int_{\mathbb{R}^{d}} \psi(y+s) p(x, d y)=\int_{\mathbb{R}^{d}} \psi(y) p(x+s, d y)
$$

for any bounded Borel function $\psi$, for any $s \in \mathbb{Z}^{d}$,

iii. $\nu(d x)$ is Markov-stationary in the following sense

$$
\iint_{\mathbb{R}^{d} \times \mathbb{R}^{d}} \psi(y) p(x, d y) \nu(d x)=\int_{\mathbb{R}^{d}} \psi(y) \nu(d y)
$$

for any bounded Borel function $\psi$.

The sigma-finite Markov chain $\hat{\mu}$ on $\Sigma$ is given as usual as

$$
\int_{\Sigma} \psi(\underline{x}) d \hat{\mu}(\underline{x})=\int_{\mathbb{R}^{d}} \cdots \int_{\mathbb{R}^{d}} \psi\left(x_{0}, x_{1}, \ldots, x_{n}\right) \nu\left(d x_{0}\right) p\left(x_{0}, d x_{1}\right) \cdots p\left(x_{n-1} d x_{n}\right),
$$

for any bounded Borel function $\psi(\underline{x})=\psi\left(x_{0}, x_{1}, \ldots, x_{n}\right)$, for any $n \geq 0$. Then $\hat{\mu}$ is both invariant with respect to the $\mathbb{Z}^{d}$ and the shift $\sigma$ action.

The correspondence between the notions of normalized invariant Markov chains and of holonomic measures may be explained through another notion called normalized invariant transshipment measure as in Evans and Gomes [19]. 
Definition 3.3. We call normalized invariant transshipment measure $\pi$ a sigmafinite measure defined on the Borel sets of $\mathbb{R}^{d} \times \mathbb{R}^{d}$ verifying the following properties:

$i . \pi$ is invariant under the diagonal action of $\mathbb{Z}^{d}$ and has mass one on any fundamental domain,

ii. if $p r^{1}: \mathbb{R}^{d} \times \mathbb{R}^{d} \rightarrow \mathbb{R}^{d}$ and $p r^{2}: \mathbb{R}^{d} \times \mathbb{R}^{d} \rightarrow \mathbb{R}^{d}$ denote the two canonical projections, then $p r_{*}^{1}(\pi)=p r_{*}^{2}(\pi)$.

The following proposition shows the equivalence between the three notions: holonomic measures, normalized invariant transshipment measures and normalized invariant Markov chains. The proof is left to the reader and uses mainly the notion of disintegration of measures.

Proposition 3.4. The three sets of measures, holonomic measures $\mu$, normalized invariant transshipment measures $\pi$ and normalized invariant Markov chains $(\nu(d x), p(x, d y))$ are in one-to-one correspondence. The correspondence is given by:

$$
\begin{aligned}
& \iint_{\mathbb{R}^{d} \times \mathbb{R}^{d}} \psi(x, y) \pi(d x, d y):=\iint_{\mathbb{R}^{d} \times \mathbb{R}^{d}} \psi(x, x+\tau v) \mu(d x, d v), \\
& \int_{\mathbb{R}^{d}} \phi(x)\left(\int_{\mathbb{R}^{d}} \psi(x, y) p(x, d y)\right) \nu(d x):=\iint_{\mathbb{R}^{d} \times \mathbb{R}^{d}} \phi(x) \psi(x, y) \pi(d x, d y), \\
& \iint_{\mathbb{R}^{d} \times \mathbb{R}^{d}} \phi(x, v) \mu(d x, d v):=\iint_{\mathbb{R}^{d} \times \mathbb{R}^{d}} \phi\left(x, \frac{y-x}{\tau}\right) p(x, d y) \nu(d x),
\end{aligned}
$$

where $\mu(d x, d v)$ has been extended to $\mathbb{R}^{d} \times \mathbb{R}^{d}$ by invariance under the action of $\mathbb{Z}^{d}$ on the first factor.

As in the weak Mather's approach, we are interested in finding particular minimizing configurations which are located in the support of minimizing holonomic measures. We thus introduce a similar concept equivalent to Mañé's definition of critical value.

Definition 3.5. Let $L(x, v): \mathbb{T}^{d} \times \mathbb{R}^{d} \rightarrow \mathbb{R}$ be a continuous coercive Lagrangian. We call minimizing holonomic value of $L$ the quantity

$$
\bar{L}(\tau):=\inf _{\mu} \iint_{\mathbb{T}^{d} \times \mathbb{R}^{d}} L(x, v) \mu(d x, d v),
$$

where the infimum is taken over the set of holonomic measures. A measure $\mu$ attaining the infimum is called a minimizing holonomic measure.

Remark 3.6. The three equivalent definitions given in proposition 3.4 show that any holonomic measure $\mu$ seen on $\mathbb{T}^{d} \times \mathbb{R}^{d}$ can be lifted to a shift-invariant probability measure $\hat{\mu}$ on $\Sigma / \sim$ obtained from the normalized invariant Markov chain $(\nu(d x), p(x, d y))$. Conversely, the projection $\mu=\left(\Pi_{\tau}\right)_{*}(\hat{\mu})$ of any shift-invariant probability measure $\hat{\mu}$ on $\Sigma / \sim$ is holonomic:

$$
\begin{aligned}
\iint_{\mathbb{T}^{d} \times \mathbb{R}^{d}} \phi(x+\tau v) \mu(d x, d v)=\int_{\Sigma / \sim} \phi\left(x_{1}\right) \hat{\mu}(d \underline{x})= \\
\quad=\int_{\Sigma / \sim} \phi\left(x_{0}\right) \hat{\mu}(d \underline{x})=\iint_{\mathbb{T}^{d} \times \mathbb{R}^{d}} \phi(x) \mu(d x, d v) .
\end{aligned}
$$


From proposition 3.4, the minimizing holonomic value of $L$ may be computed using two different ways

$$
\bar{L}(\tau)=\inf _{\pi} \iint_{\mathbb{R}^{d} \times \mathbb{R}^{d} / \sim} L\left(x, \frac{y-x}{\tau}\right) \pi(d x, d y)=\inf _{\hat{\mu}} \int_{\Sigma / \sim} L\left(x_{0}, \frac{x_{1}-x_{0}}{\tau}\right) \hat{\mu}(d \underline{x}),
$$

where the infimums are taken, respectively, over the set of normalized invariant transshipment measures $\pi$ and over the set of shift-invariant probability measures on $\Sigma / \sim$.

Since $\mathbb{T}^{d} \times \mathbb{R}^{d}$ is not compact, the existence of a minimizing holonomic measure is not guarantee at first sight. Nevertheless, periodicity in $x$ and coerciveness in $y-x$ implies the existence of such minimizing measures.

Proposition 3.7. Let $L(x, v): \mathbb{T}^{d} \times \mathbb{R}^{d} \rightarrow \mathbb{R}$ be a continuous coercive Lagrangian. Then there exists a minimizing holonomic measure having a compact support.

Before going into the proof of this result, we will make use of a special piecewise continuous map $F_{\tau}: \mathbb{T}^{d} \times \mathbb{R}^{d} \rightarrow \mathbb{T}^{d} \times \mathbb{R}^{d}$ which enables us to replace $\mathbb{R}^{d}$ by a compact ball. Let $\|v\|_{\infty}=\max _{i}\left|v_{i}\right|$ be the maximum norm.

Definition 3.8. Suppose $L(x, v)$ is a coercive Lagrangian, then there exists a real number $R_{\tau}>1 / \tau$ such that

$$
\inf _{\|v\|_{\infty} \geq R_{\tau}} \inf _{x \in \mathbb{T}^{d}} L(x, v)>\sup _{\|v\|_{\infty} \leq 1 / \tau} \sup _{x \in \mathbb{T}^{d}} L(x, v) .
$$

Let $\lfloor v\rfloor \in \mathbb{Z}^{d}$ denotes the vector whose coordinates are the greatest integers less or equal than the respective coordinates of $v \in \mathbb{R}^{d}$.

Lemma 3.9. Let $L(x, v)$ be a $C^{0}$ coercive Lagrangian and $F_{\tau}: \mathbb{T}^{d} \times \mathbb{R}^{d} \rightarrow \mathbb{T}^{d} \times \mathbb{R}^{d}$ defined by

$$
F_{\tau}(x, v)=\left\{\begin{array}{ll}
\left(x, v-\frac{1}{\tau}\lfloor\tau v\rfloor\right) & \text { if }\|v\|_{\infty} \geq R_{\tau} \\
(x, v) & \text { if }\|v\|_{\infty}<R_{\tau}
\end{array} .\right.
$$

Then $F_{\tau}$ satisfies

i. the image $F_{\tau}\left(\mathbb{T}^{d} \times \mathbb{R}^{d}\right)$ is a bounded set;

ii. $L(x, v) \geq L \circ F_{\tau}(x, v) \quad \forall(x, v) \in \mathbb{T}^{d} \times \mathbb{R}^{d} ;$

iii. $\mu \in \mathcal{P}_{\tau}\left(\mathbb{T}^{d} \times \mathbb{R}^{d}\right) \Rightarrow\left(F_{\tau}\right)_{*} \mu \in \mathcal{P}_{\tau}\left(\mathbb{T}^{d} \times \mathbb{R}^{d}\right)$.

Proof. The first item is obviously satisfied. The second one is just a consequence of the choice of $R_{\tau}$. Finally, since $\psi(x+\tau v-\lfloor\tau v\rfloor)=\psi(x+\tau v)$ for every $\psi \in C^{0}\left(\mathbb{T}^{d}\right)$, the third item follows without difficulty.

We can now prove the existence of minimizing holonomic measures for $C^{0}$ coercive Lagrangians.

Proof of proposition 3.7. Consider a sequence $\left\{\mu_{n}\right\} \subset \mathcal{P}_{\tau}\left(\mathbb{T}^{d} \times \mathbb{R}^{d}\right)$ of holonomic measures satisfying $\lim _{n} \int L(x, v) d \mu_{n}(x, v)=\bar{L}(\tau)$. Items $i i$ and $i i i$ of lemma 3.9 assure that the sequence $\left\{\nu_{n}=\left(F_{\tau}\right)_{*} \mu_{n}\right\}$ verifies the same properties. Furthermore, by item $i$ of the same lemma, all probability measures $\nu_{n}$ are supported on a common compact set. Therefore, any accumulation point $\nu \in \mathcal{P}_{\tau}\left(\mathbb{T}^{d} \times \mathbb{R}^{d}\right)$ of $\left\{\nu_{n}\right\}$ for the narrow topology satisfies $\int L(x, v) d \nu(x, v)=\bar{L}(\tau)$. 


\section{Lax-Oleinik operators}

The Lax-Oleinik semigroup is well known in partial differential equations and in calculus of variations. It was used by A. Fathi (see [21]) for obtaining the so-called weak KAM theorem in the framework of continuous-time, autonomous, strictly convex and superlinear $C^{3}$-Lagrangians on a compact manifold.

Several results in this section are comparable to those in D. Gomes [34]. There are some differences: in the computation of the "effective" $\bar{L}(\tau)$, we do not use the abstract Fenchel-Rockafellar duality theorem to prove the sup-inf principle (proposition 4.5 in this section or theorem 2.3 in[34]); we do not use a penalized Lax-Oleinik version as in [34] which corresponds to a more numerical approach (theorem 4.3 in this section or theorem 3.3 in [34]). More important, we are carefull about the degree of minimum regularity we need in each step; we give in particular two estimates of regularity which are very comparable to those in D. Gomes but with different hypothesis (proposition 4.7 and lemma 6.8 in this paper or propositions 5.1 and 5.2 in [34]).

In our context, we are interested in studying operators with similar properties to the Lax-Oleinik semigroup. We recall that $\mathcal{L}_{\tau}(x, y)=\tau L\left(x, \frac{y-x}{\tau}\right)$.

Definition 4.1. Given a $C^{0}$ coercive Lagrangian $L=L(x, v): \mathbb{T}^{d} \times \mathbb{R}^{d} \rightarrow \mathbb{R}$ and a constant $\tau>0$, we call forward and backward Lax-Oleinik operators, respectively, the maps $T_{+}$and $T_{-}$defined by

$$
\begin{aligned}
& T_{+} u(x)=\sup _{v \in \mathbb{R}^{d}}[u(x+\tau v)-\tau L(x, v)]=\sup _{y \in \mathbb{R}^{d}}\left[u(y)-\mathcal{L}_{\tau}(x, y)\right], \\
& T_{-} u(y)=\inf _{v \in \mathbb{R}^{d}}[u(y-\tau v)+\tau L(y-\tau v, v)]=\inf _{x \in \mathbb{R}^{d}}\left[u(y)+\mathcal{L}_{\tau}(x, y)\right],
\end{aligned}
$$

for every $\mathbb{Z}^{d}$-periodic function $u \in C^{0}\left(\mathbb{R}^{d}\right)$ that we identify with $u \in C^{0}\left(\mathbb{T}^{d}\right)$.

Because of the choice of $R_{\tau}$ in definition 3.8 and the fact that the minimization of $L$ can be made on the ball $\|v\|_{\infty} \leq R_{\tau}$ as explained in lemma 3.9, $T_{ \pm}$are well defined and have the following more restricted definition

$$
\begin{gathered}
T_{+} u(x)=\max _{\|v\|_{\infty} \leq R_{\tau}}[u(x+\tau v)-\tau L(x, v)]=\max _{\|y-x\|_{\infty} \leq \tau R_{\tau}}\left[u(y)-\mathcal{L}_{\tau}(x, y)\right], \\
T_{-} u(y)=\min _{\|v\|_{\infty} \leq R_{\tau}}[u(y-\tau v)+\tau L(y-\tau v, v)]=\min _{\|y-x\|_{\infty} \leq \tau R_{\tau}}\left[u(y)+\mathcal{L}_{\tau}(x, y)\right] .
\end{gathered}
$$

Such identities are immediate consequences of the explicit construction of the application $F_{\tau}: \mathbb{T}^{d} \times \mathbb{R}^{d} \rightarrow \mathbb{T}^{d} \times \mathbb{R}^{d}$ whose properties are described in lemma 3.9. Indeed, writing

$$
\phi_{+}(x, v)=u(x+\tau v)-\tau L(x, v) \text { and } \phi_{-}(x, v)=u(x-\tau v)+\tau L(x-\tau v, v),
$$

we get $\phi_{+} \circ F_{\tau} \geq \phi_{+}$and $\phi_{-} \circ F_{\tau} \leq \phi_{-}$. So we have

$$
\max _{v \in \mathbb{R}^{d}} \phi_{+}(x, v)=\max _{v \in \mathbb{R}^{d}} \phi_{+} \circ F_{\tau}(x, v)=\max _{\|v\|_{\infty} \leq R_{\tau}} \phi_{+}(x, v),
$$

and similar equalities for $\phi_{-}$as well.

Let $\operatorname{osc}(f, D)$ denote the oscillation of a function $f$ on a subset $D$ of its domain. 
Lemma 4.2. Let $L(x, v)$ be a $C^{0}$ coercive Lagrangian. Then the Lax-Oleinik operators verify the following properties.

i. For all $u \in C^{0}\left(\mathbb{T}^{d}\right)$, for all $x, y \in \mathbb{T}^{d}$,

$$
\begin{gathered}
\left|T_{+} u(x)-T_{+} u(y)\right| \leq \max _{v^{*}, w^{*}} \tau\left|L\left(x, v^{*}\right)-L\left(y, w^{*}\right)\right| \text { and } \\
\left|T_{-} u(x)-T_{-} u(y)\right| \leq \max _{v^{*}, w^{*}} \tau\left|L\left(x-\tau v^{*}, v^{*}\right)-L\left(y-\tau w^{*}, w^{*}\right)\right|,
\end{gathered}
$$

where the maxima are taken over

$$
\left\|v^{*}\right\|_{\infty},\left\|w^{*}\right\|_{\infty} \leq 2 R_{\tau} \quad \text { and } \quad\left\|v^{*}-w^{*}\right\|_{\infty} \leq \frac{\|x-y\|_{\infty}}{\tau} .
$$

ii. The two operators $T_{+}$and $T_{-}$map $C^{0}\left(\mathbb{T}^{d}\right)$ into itself.

iii. The two sets $T_{+}\left(C^{0}\left(\mathbb{T}^{d}\right)\right)$ and $T_{-}\left(C^{0}\left(\mathbb{T}^{d}\right)\right)$ are equicontinuous.

In particular, $\operatorname{osc}\left(T_{+} u, \mathbb{T}^{d}\right)$ and $\operatorname{osc}\left(T_{-} u, \mathbb{T}^{d}\right)$ are bounded by the oscillation of $\tau L$ on $\mathbb{T}^{d} \times B_{2 R_{\tau}}$, where $B_{2 R_{\tau}}$ denotes the closed ball of center 0 and radius $2 R_{\tau}$.

Proof. On the one hand, for any point $x$ in $\mathbb{R}^{d}$, there exists $z^{*} \in \mathbb{R}^{d}$, such that $\left\|x-z^{*}\right\|_{\infty} \leq \tau R_{\tau}$ and $T_{+} u(x)=u\left(z^{*}\right)-\mathcal{L}_{\tau}\left(x, z^{*}\right)$. On the other hand, for any $y \in \mathbb{R}^{d}, T_{+} u(y) \geq u\left(z^{*}\right)-\mathcal{L}_{\tau}\left(y, z^{*}\right)$. Combining these two estimates, we obtain

$$
T_{+} u(y)-T_{+} u(x) \geq \mathcal{L}_{\tau}\left(x, z^{*}\right)-\mathcal{L}_{\tau}\left(y, z^{*}\right)=\tau\left[L\left(x, v^{*}\right)-L\left(y, w^{*}\right)\right]
$$

where $w^{*}=v^{*}+\frac{x-y}{\tau}$ and $z^{*}=x+\tau v^{*}$. A similar estimate holds by permuting $x$ and $y$ which proves the first property for $T_{+}$. An analogous argument can be used to demonstrate the inequality concerning the backward Lax-Oleinik operator $T_{-}$. Since $L(x, v)$ is uniformly continuous on $\mathbb{T}^{d} \times B_{2 R_{\tau}}$, the two sets $T_{ \pm}\left(C^{0}\left(\mathbb{T}^{d}\right)\right)$ are equicontinuous and the lemma is proved.

We recall that the minimizing holonomic value $\bar{L}(\tau)$ has been introduced in definition 3.5. So the main theorem of this section can be stated as follows.

Theorem 4.3. If $L(x, v)$ is a $C^{0}$ coercive Lagrangian, then there exist continuous periodic solutions of the Lax-Oleinik equation, $u_{+}, u_{-} \in C^{0}\left(\mathbb{T}^{d}\right)$, satisfying

$$
T_{+} u_{+}=u_{+}-\tau \bar{L}(\tau) \quad \text { and } \quad T_{-} u_{-}=u_{-}+\tau \bar{L}(\tau) .
$$

Moreover $u_{ \pm}$satisfies the a priori estimate: $\left\|u_{+}\right\|_{0},\left\|u_{-}\right\|_{0} \leq \operatorname{osc}\left(\tau L, \mathbb{T}^{d} \times B_{2 R_{\tau}}\right)$.

Proof. If we equip $C^{0}\left(\mathbb{T}^{d}\right)$ with the topology of the uniform convergence, it is easy to show that $T_{+}: C^{0}\left(\mathbb{T}^{d}\right) \rightarrow C^{0}\left(\mathbb{T}^{d}\right)$ is 1-Lipschitz. So the Lipschitz regularity is also respected by the application $\hat{T}_{+}: C^{0}\left(\mathbb{T}^{d}\right) \rightarrow C^{0}\left(\mathbb{T}^{d}\right)$ defined by

$$
\hat{T}_{+} u=T_{+} u-\max \left(T_{+} u\right) .
$$

Obviously $\hat{T}_{+} u \leq 0$ everywhere on $C^{0}\left(\mathbb{T}^{d}\right)$. Conversely, it follows from lemma 4.2 that

$$
\hat{T}_{+} u \geq-\operatorname{osc}\left(\tau L, \mathbb{T}^{d} \times B_{2 R_{\tau}}\right), \quad \forall u \in C^{0}\left(\mathbb{T}^{d}\right) .
$$


Let $\mathfrak{B} \subset C^{0}\left(\mathbb{T}^{d}\right)$ denote the closed convex hull of the closure of $\hat{T}_{+}\left(C^{0}\left(\mathbb{T}^{d}\right)\right)$. Since the image $\hat{T}_{+}\left(C^{0}\left(\mathbb{T}^{d}\right)\right)$ is bounded, $\mathfrak{B}$ is a compact convex set. As $\hat{T}_{+}(\mathfrak{B}) \subset \mathfrak{B}$, by the Schauder-Tychonoff fixed point theorem, there exists a function $u_{+} \in C^{0}\left(\mathbb{T}^{d}\right)$ such that

$$
T_{+} u_{+}=u_{+}+\max \left(T_{+} u_{+}\right) .
$$

Obviously, $\left\|u_{+}\right\|_{0}=\left\|\hat{T}_{+}\left(u_{+}\right)\right\|_{0} \leq \operatorname{osc}\left(\tau L, \mathbb{T}^{d} \times B_{2 R_{\tau}}\right)$. It remains to show that $\max \left(T_{+} u_{+}\right)=-\tau \bar{L}(\tau)$. On the one hand

$$
\tau L(x, v)+u_{+}(x)-u_{+}(x+\tau v) \geq-\max \left(T_{+} u_{+}\right)
$$

everywhere on $\mathbb{T}^{d} \times \mathbb{R}^{d}$. For any holonomic probability measure $\mu \in \mathcal{P}_{\tau}\left(\mathbb{T}^{d} \times \mathbb{R}^{d}\right)$, by integrating the previous inequality, we obtain

$$
\tau \int L(x, v) d \mu=\int\left[\tau L(x, v)+u_{+}(x)-u_{+}(x+\tau v)\right] d \mu \geq-\max \left(T_{+} u_{+}\right)
$$

and therefore $\max \left(T_{+} u_{+}\right) \geq-\tau \bar{L}(\tau)$.

On the other hand, given $x_{0} \in \mathbb{T}^{d}$, there exists a vector $v_{0} \in \mathbb{R}^{d}$ such that $\left\|v_{0}\right\|_{\infty} \leq R_{\tau}$ and $\tau L\left(x_{0}, v_{0}\right)+u_{+}\left(x_{0}\right)-u_{+}\left(x_{0}+\tau v_{0}\right)=-\max \left(T_{+} u_{+}\right)$. For every $k \geq 1$, if $x_{k}=x_{k-1}+\tau v_{k-1} \in \mathbb{T}^{d}$, we consider inductively $v_{k} \in \mathbb{R}^{d}$ such that $\left\|v_{k}\right\| \leq R_{\tau}$ and $\tau L\left(x_{k}, v_{k}\right)+u_{+}\left(x_{k}\right)-u_{+}\left(x_{k}+\tau v_{k}\right)=-\max \left(T_{+} u_{+}\right)$. Let $\left\{\mu_{n}\right\}$ be the probability measure defined by

$$
\mu_{n}=\frac{1}{n} \sum_{k=0}^{n-1} \delta_{\left(x_{k}, v_{k}\right)} .
$$

Since their supports are contained in the compact set $\mathbb{T}^{d} \times B_{R_{\tau}}$, such a sequence is relatively compact for the narrow topology. Let $\mu \in \mathcal{P}_{\tau}\left(\mathbb{T}^{d} \times \mathbb{R}^{d}\right)$ be some convergent subsequence limit. Note that the equality

$$
\int\left[\tau L(x, v)+u_{+}(x)-u_{+}(x+\tau v)\right] d \mu_{n}(x, v)=-\max T_{+} u_{+},
$$

goes through the limit $\mu$. Hence, we obtain $\max T_{+} u_{+} \leq-\tau \bar{L}(\tau)$ if we prove that $\mu$ is a holonomic probability measure. Indeed, for any function $\psi \in C^{0}\left(\mathbb{T}^{d}\right)$,

$$
\begin{aligned}
\left|\int[\psi(x+\tau v)-\psi(x)] d \mu_{n}(x, v)\right| & =\frac{1}{n}\left|\sum_{k=0}^{n-1}\left[\psi\left(x_{k}+\tau v_{k}\right)-\psi\left(x_{k}\right)\right]\right| \\
& =\frac{1}{n}\left|\psi\left(x_{n}\right)-\psi\left(x_{0}\right)\right| \leq \frac{2}{n}\|\psi\|_{0} .
\end{aligned}
$$

Letting $n$ go to infinity, we immediately obtain that $\mu \in \mathcal{P}_{\tau}\left(\mathbb{T}^{d} \times \mathbb{R}^{d}\right)$. The existence of a function $u_{-} \in C^{0}\left(\mathbb{T}^{d}\right)$ is obtained in an analogous way.

The following result is an immediate consequence of the previous proof.

Corollary 4.4. Let $L(x, v)$ be a $C^{0}$ coercive Lagrangian. If $u \in C^{0}\left(\mathbb{T}^{d}\right)$ satisfies either $T_{+} u=u-c$ or $T_{-} u=u+c$ for some constant $c \in \mathbb{R}$, then $c=\tau \bar{L}(\tau)$. 
The previous theorem 4.3 may be seen as an important theorical tool: it gives a way to renormalize the initial Lagrangian by a coboundary

$$
L_{\mathrm{norm}}(x, v)=L(x, v)-\bar{L}(\tau)-\frac{1}{\tau}[u(x+\tau v)-u(x)] \geq 0, \quad \forall(x, v) \in \mathbb{T}^{d} \times \mathbb{R}^{d} .
$$

The existence of a solution of the Lax-Oleinik operator also gives other characterizations of the minimizing holonomic value, either as a max-min optimal value or as an ergodic average asymptotic value.

Proposition 4.5. Let $L(x, v)$ be a $C^{0}$ coercive Lagrangian. Then we have

or

$$
\tau \bar{L}(\tau)=\sup _{\psi \in C^{0}\left(\mathbb{T}^{d}\right)} \inf _{(x, v) \in \mathbb{T}^{d} \times \mathbb{R}^{d}}[\tau L(x, v)+\psi(x)-\psi(x+\tau v)]
$$

$$
\tau \bar{L}(\tau)=\inf _{\left\{x_{k}\right\} \in\left(\mathbb{R}^{d}\right)^{\mathbb{Z}_{+}}} \liminf _{n \rightarrow \infty} \frac{1}{n} \sum_{k=0}^{n-1} \mathcal{L}_{\tau}\left(x_{k}, x_{k+1}\right) .
$$

Proof. Let $\mu \in \mathcal{P}_{\tau}\left(\mathbb{T}^{d} \times \mathbb{R}^{d}\right)$ be any minimizing holonomic measure. Then

$$
\begin{aligned}
\tau \bar{L}(\tau) & =\int \tau L(x, v) d \mu(x, v) \\
& =\int[\tau L(x, v)+\psi(x)-\psi(x+\tau v)] d \mu(x, v) \\
& \geq \inf _{(x, v) \in \mathbb{T}^{d} \times \mathbb{R}^{d}}[\tau L(x, v)+\psi(x)-\psi(x+\tau v)]
\end{aligned}
$$

for every $\psi \in C^{0}\left(\mathbb{T}^{d}\right)$. By taking the supremum over all $\psi \in C^{0}\left(\mathbb{T}^{d}\right)$, one obtain a lower bound of $\tau \bar{L}(\tau)$. Conversely, theorem 4.3 establishes there is $u_{+} \in C^{0}\left(\mathbb{T}^{d}\right)$ such that

$$
\tau \bar{L}(\tau)=\inf _{(x, v) \in \mathbb{T}^{d} \times \mathbb{R}^{d}}\left[\tau L(x, v)+u_{+}(x)-u_{+}(x+\tau v)\right] .
$$

The first identity is proved. Consider now an arbitrary sequence $\left\{x_{k}\right\} \in\left(\mathbb{R}^{d}\right)^{\mathbb{Z}_{+}}$. Then for any $n>0$

$$
n \bar{L}(\tau) \leq \sum_{k=0}^{n-1} \mathcal{L}_{\tau}\left(x_{k}, x_{k+1}\right)+u_{+}\left(x_{0}\right)-u_{+}\left(x_{n}\right) \leq \sum_{k=0}^{n-1} \mathcal{L}_{\tau}\left(x_{k}, x_{k+1}\right)+2\left\|u_{+}\right\|_{0} .
$$

Dividing by $n$ and letting $n$ go to infinity, we obtain the above upper bound for $\tau \bar{L}(\tau)$. Conversely, choose any optimal sequence $\left\{x_{k}^{*}\right\}_{k \geq 0}$ in $\mathbb{R}^{d}$ such that

$$
\tau \bar{L}(\tau)=\mathcal{L}_{\tau}\left(x_{k}^{*}, x_{k+1}^{*}\right)+u_{+}\left(x_{k}^{*}\right)-u_{+}\left(x_{k+1}^{*}\right), \quad \forall k \geq 0 .
$$

Dividing again by $n$ and letting $n$ go to infinity, we then obtain the above lower bound for $\tau \bar{L}(\tau)$ and the second identity is proved.

Thanks to theorem 4.3, we know that the solutions $u_{ \pm}$of the Lax-Oleinik operator are continuous. If in addition $L$ is locally $\alpha$-Hölder continuous, the same estimate of part $i$ in lemma 4.2 shows that $u_{ \pm}$is also $\alpha$-Hölder continuous. In fact, these solutions possess a stronger regularity if $L$ is supposed to be semiconcave. 
Definition 4.6. A function $F: \mathbb{T}^{d} \times \mathbb{R}^{d} \rightarrow \mathbb{R}$ is called semiconcave if, for every $R>0$, there exists a nondecreasing upper semicontinuous function $\theta_{R}: \mathbb{R}_{+} \rightarrow \mathbb{R}_{+}$ satisfying $\lim _{\rho \rightarrow 0^{+}} \theta_{R}(\rho)=0$ and

$$
t F(\xi)+(1-t) F(\eta)-F(t \xi+(1-t) \eta) \leq t(1-t)\|\xi-\eta\| \theta_{R}(\|\xi-\eta\|)
$$

for all $\xi=(x, v), \eta=(y, w)$ in $\mathbb{R}^{d} \times \mathbb{R}^{d}$ with $\|v\|,\|w\| \leq R$ and for any $t \in[0,1]$. We call $\left\{\theta_{R}\right\}_{R>0}$ a family of local modulus of semiconcavity for $F$. A function $G: \mathbb{T}^{d} \times \mathbb{R}^{d} \rightarrow \mathbb{R}$ is called semiconvex if $-G$ is semiconcave.

Notice that in the case the function $F(x)$ depends only in $x \in \mathbb{T}^{d}$, semiconcavity is defined using a unique modulus $\theta$ instead of a family $\left\{\theta_{R}\right\}_{R>0}$. Any $C^{2}$-Lagrangian $L(x, v)$ is an example of a semiconcave function with modulus of semiconcavity $\theta_{R}(\rho)=C_{R} \rho$ and $C_{R}:=\frac{1}{2} \max _{x \in \mathbb{T}^{d}} \max _{\|v\| \leq R}\|\operatorname{Hess}(L)(x, v)\|_{\infty}$. The proof is standard.

For more details on semiconcave functions, we refer the reader to the book of $\mathrm{P}$. Cannarsa and C. Sinestrari (see [14]). Let us examine how the forward Lax-Oleinik operator $T_{+}$deals with semiconcavity.

Proposition 4.7. Let $L(x, v)$ be a semiconcave $C^{0}$ coercive Lagrangian. Then any solution $u \in C^{0}\left(\mathbb{T}^{d}\right)$ of the forward Lax-Oleinik equation, $T_{+} u=u-\tau \bar{L}(\tau)$, is semiconvex.

Proof. Given $x, y \in \mathbb{R}^{d}$ and $t \in[0,1]$, set $z=t x+(1-t) y$. Then there exists an optimal $z^{*} \in \mathbb{R}^{d}$ such that

$$
u(z)=u\left(z^{*}\right)-\mathcal{L}_{\tau}\left(z, z^{*}\right)+\tau \bar{L}(\tau)
$$

with $\left\|z-z^{*}\right\|_{\infty} \leq \tau R_{\tau}$. Moreover

$$
u(x) \leq u\left(z^{*}\right)-\mathcal{L}_{\tau}\left(x, z^{*}\right)+\tau \bar{L}(\tau) \text { and } u(y) \leq u\left(z^{*}\right)-\mathcal{L}_{\tau}\left(y, z^{*}\right)+\tau \bar{L}(\tau) .
$$

Combining these two inequalities and the previous identity, we obtain

$$
t u(x)+(1-t) u(y)-u(z) \geq-\left[t \mathcal{L}_{\tau}\left(x, z^{*}\right)+(1-t) \mathcal{L}_{\tau}\left(y, z^{*}\right)-\mathcal{L}_{\tau}\left(z, z^{*}\right)\right] .
$$

Let $v^{*}$ and $w^{*}$ be defined by $z^{*}=x+\tau v^{*}$ and $z^{*}=y+\tau w^{*}$. Hence

$$
z^{*}=z+\tau\left(t v^{*}+(1-t) w^{*}\right) \quad \text { and } \quad\left\|v^{*}\right\|_{\infty},\left\|w^{*}\right\|_{\infty} \leq R_{\tau}+\left\|\frac{x-y}{\tau}\right\|_{\infty} \leq 2 R_{\tau} .
$$

Then

$$
\begin{aligned}
& t u(x)+(1-t) u(y)-u(z) \geq \\
& \geq-\tau\left[t L\left(x, v^{*}\right)+(1-t) L\left(y, w^{*}\right)-L\left(z, t v^{*}+(1-t) w^{*}\right)\right] \geq \\
& \geq-\tau t(1-t) \frac{2}{\tau}\|x-y\|_{\infty} \theta_{2 R_{\tau}}\left(\frac{2}{\tau}\|x-y\|_{\infty}\right)
\end{aligned}
$$

using the fact that $\left\|v^{*}-w^{*}\right\|_{\infty}=\frac{1}{\tau}\|x-y\|_{\infty}$. We have shown that $\frac{1}{\tau} u$ is semiconvex with a modulus of convexity $\theta(\rho)=-\frac{2}{\tau} \theta_{2 R_{\tau}}\left(\frac{2}{\tau} \rho\right)$.

Similarly, any solution $u$ of the backward Lax-Oleinik equation $T_{-} u=u+\tau \bar{L}(\tau)$ is semiconcave as soon as $L(x, v)$ is a semiconcave $C^{0}$ coercive Lagrangian. 


\section{Calibrated and minimizing configurations}

For conciseness, for any given configuration $\left\{x_{k}\right\}_{k \in \mathbb{Z}}$ of points in $\mathbb{R}^{d}$, for any $m<n$, we call normalized interaction energy of a finite configuration the quantity

$$
\overline{\mathcal{L}}_{\tau}\left(x_{m}, x_{m+1}, \ldots, x_{n}\right):=\sum_{k=m}^{n-1}\left[\mathcal{L}_{\tau}\left(x_{k}, x_{k+1}\right)-\tau \bar{L}(\tau)\right] .
$$

Let us recall from the introduction the fundamental definition of mimimizing configurations.

Definition 5.1. Consider a bounded below $C^{0}$-Lagrangian $L(x, v)$. We say that a configuration $\left\{x_{k}\right\}_{k \in \mathbb{Z}}$ of points of $\mathbb{R}^{d}$ is a minimizing configuration if, for every pair $m<n$,

$$
\overline{\mathcal{L}}_{\tau}\left(x_{m}, x_{m+1}, \ldots, x_{n}\right) \leq \overline{\mathcal{L}}_{\tau}\left(y_{m}, y_{m+1}, \ldots, y_{n}\right)
$$

whenever $\left\{y_{k}\right\}_{k \in \mathbb{Z}}$ satisfies $y_{m}=x_{m}$ and $y_{n}=x_{n}$. A configuration $\left\{x_{k}\right\}_{k \in \mathbb{Z}}$ is called strongly minimizing configuration if, for any two pairs $m<n, m^{\prime}<n^{\prime}$ and any configuration $\left\{y_{k}\right\}_{k \in \mathbb{Z}}$ satisfying $y_{m^{\prime}}=x_{m}$ and $y_{n^{\prime}}=x_{n}\left(\bmod \mathbb{Z}^{d}\right)$, we have

$$
\overline{\mathcal{L}}_{\tau}\left(x_{m}, x_{m+1}, \ldots, x_{n}\right) \leq \overline{\mathcal{L}}_{\tau}\left(y_{m^{\prime}}, y_{m^{\prime}+1}, \ldots, y_{n^{\prime}}\right) .
$$

For a coercive Lagrangian, notice that definition 3.8 implies consecutive jumps $x_{k+1}-x_{k}$ are uniformly bounded for strongly minimizing configurations $\left\{x_{k}\right\}_{k \in \mathbb{Z}}$, namely,

$$
\sup _{k \in \mathbb{Z}}\left\|x_{k+1}-x_{k}\right\|_{\infty}<\tau R_{\tau} .
$$

In order to introduce an important class of minimizing configurations, we will need to consider the following notions.

Definition 5.2. Let $L(x, v)$ be a $C^{0}$ coercive Lagrangian. A function $u: \mathbb{R}^{d} \rightarrow \mathbb{R}$ is a called sub-action (or $\tau$-sub-action if necessary) with respect to $L$ if $u(x)$ is $\mathbb{Z}^{d}$-periodic, continuous and satisfies

$$
\tau \bar{L}(\tau) \leq \tau L(x, v)+u(x)-u(x+\tau v), \quad \forall(x, v) \in \mathbb{T}^{d} \times \mathbb{R}^{d} .
$$

More restrictively, $u$ is called forward calibrated if it is a sub-action and

$$
u(x)+\tau \bar{L}(\tau)=\sup _{v \in \mathbb{R}^{d}}[u(x+\tau v)-\tau L(x, v)], \quad \forall x \in \mathbb{T}^{d},
$$

and similarly $u$ is called backward calibrated if it is a sub-action and

$$
u(x)+\tau \bar{L}(\tau)=\inf _{v \in \mathbb{R}^{d}}[u(x-\tau v)+\tau L(x-\tau v, v)], \quad \forall x \in \mathbb{T}^{d} .
$$

Notice that the $C^{0}$ periodic functions $u_{+}, u_{-}$are forward or backward calibrated sub-actions if, and only if, they are solutions of the forward or backward Lax-Oleinik equations given in theorem 4.3. The existence of sub-actions has been proved under the sole hypothesis of coerciveness. 
Proposition 5.3. Let $L(x, v)$ be a $C^{0}$ coercive Lagrangian. If $L$ is locally $\alpha$-Hölder continuous, then any forward or backward calibrated sub-action $u \in C^{0}\left(\mathbb{T}^{d}\right)$ is $\alpha$ Hölder continuous too. Besides, if $L$ is semiconcave, then all forward calibrated sub-actions are semiconvex and all backward calibrated sub-actions are semiconcave.

Proof. The Hölder case is an immediate consequence of part $i$ of lemma 4.2. The semiconvex property is just a reinterpretation of proposition 4.7 as well as its analogue for backward calibrated sub-actions mentioned after its demonstration.

Observe that, in terms of the associated local interaction energy $\mathcal{L}_{\tau}(x, y)$, a sub-action $u$ satisfies $\mathcal{L}_{\tau}(x, y) \geq u(y)-u(x)+\tau \bar{L}(\tau)$ everywhere on $\mathbb{R}^{d} \times \mathbb{R}^{d}$.

Definition 5.4. Consider a $C^{0}\left(\mathbb{T}^{d}\right)$ sub-action $u$ for a $C^{0}$ coercive Lagrangian $L(x, v)$. A configuration $\left\{x_{k}\right\}_{k \in \mathbb{Z}}$ in $\mathbb{R}^{d}$ is called $u$-calibrated if, for every $k \in \mathbb{Z}$, we have $\mathcal{L}_{\tau}\left(x_{k}, x_{k+1}\right)=u\left(x_{k+1}\right)-u\left(x_{k}\right)+\tau \bar{L}(\tau)$.

It is easy to see that calibrated configurations are minimizing and even strongly minimizing. We show in the following lemma that the coerciveness assumption implies the existence of calibrated configurations and therefore the existence of minimizing configurations.

Lemma 5.5. Suppose $L(x, v)$ is a $C^{0}$ coercive Lagrangian. If $u \in C^{0}\left(\mathbb{T}^{d}\right)$ is either a forward or a backward calibrated sub-action, then there exists a u-calibrated configuration $\left\{x_{k}\right\}_{k \in \mathbb{Z}}$ in $\mathbb{R}^{d}$ passing through some point $x_{0} \in[0,1)^{d}$ and satisfying $\left\|x_{k+1}-x_{k}\right\|_{\infty} \leq \tau R_{\tau}$, where $R_{\tau}>\frac{1}{\tau}$ has been defined in 3.8 .

Lemma 5.6. Let $L(x, v)$ be a $C^{0}$ coercive Lagrangian. If $u \in C^{0}\left(\mathbb{T}^{d}\right)$ is an arbitrary sub-action, then any u-calibrated configuration $\left\{x_{k}\right\}_{k \in \mathbb{Z}}$ in $\mathbb{R}^{d}$ is a strongly minimizing configuration satisfying $\left\|x_{k+1}-x_{k}\right\|_{\infty} \leq \tau R_{\tau}$ for every $k \in \mathbb{Z}$.

Proof of lemma 5.5. Let $u \in C^{0}\left(\mathbb{T}^{d}\right)$ be a forward calibrated sub-action. Thanks to coerciveness, $u$ verifies $\tau \bar{L}(\tau)=\min _{\|v\|_{\infty} \leq R_{\tau}}[\tau L(x, v)+u(x)-u(x+\tau v)]$ or

$$
\tau \bar{L}(\tau)=\min _{y:\|y-x\|_{\infty} \leq \tau R_{\tau}}\left[\mathcal{L}_{\tau}(x, y)+u(x)-u(y)\right], \quad \forall x \in \mathbb{R}^{d} .
$$

Hence, for every positive integer $n$, consider a configuration $\left\{x_{k}^{n}\right\}_{k \geq-n}$ in $\mathbb{R}^{d}$ such that $\left\|x_{k}^{n}-x_{k+1}^{n}\right\|_{\infty} \leq \tau R_{\tau}$ and $\tau \bar{L}(\tau)=L_{\tau}\left(x_{k}^{n}, x_{k+1}^{n}\right)+u\left(x_{k}^{n}\right)-u\left(x_{k+1}^{n}\right)$ for all $k \geq-n$. Since $L_{\tau}(x+s, y+s)=L_{\tau}(x, y)$ for $s \in \mathbb{Z}^{d}$, we may assume that $x_{0}^{n} \in[0,1)^{d}$ for every $n>0$. In particular, we get that $\left\|x_{k}^{n}\right\|_{\infty} \leq \tau R_{\tau}|k|+1$ for all $k \geq-n$. By a diagonal procedure, we extract a configuration $\left\{x_{k}\right\}_{k \in \mathbb{Z}}$ satisfying $\tau \bar{L}(\tau)=\mathcal{L}_{\tau}\left(x_{k}, x_{k+1}\right)+u\left(x_{k}\right)-u\left(x_{k+1}\right)$ for any integer $k$. A similar proof can be developed for $C^{0}\left(\mathbb{T}^{d}\right)$ backward calibrated sub-actions.

Proof of lemma 5.6. Let $\left\{x_{k}\right\}_{k \in \mathbb{Z}}$ be a $u$-calibrated configuration. Thanks to definition 3.8 , if $\left\|x_{k+1}-x_{k}\right\|_{\infty}>\tau R_{\tau}$, then

$$
\begin{aligned}
& u\left(x_{k+1}\right)-u\left(x_{k}\right)+\tau \bar{L}(\tau)=\mathcal{L}_{\tau}\left(x_{k}, x_{k+1}\right)> \\
& \quad>\mathcal{L}_{\tau}\left(x_{k}, x_{k+1}-\left\lfloor x_{k+1}-x_{k}\right\rfloor\right) \geq u\left(x_{k+1}-\left\lfloor x_{k+1}-x_{k}\right\rfloor\right)-u\left(x_{k}\right)+\tau \bar{L}(\tau) .
\end{aligned}
$$


The periodicity of $u$ implies that the strict inequality cannot happen. Therefore, $\left\|x_{k+1}-x_{k}\right\|_{\infty} \leq \tau R_{\tau}$ for all $k \in \mathbb{Z}$. Moreover, for any $m<n$, for any configuration $\left\{y_{k}\right\}_{k \in \mathbb{Z}}$ in $\mathbb{R}^{d}$ satisfying $y_{m^{\prime}}=x_{m}$ and $y_{n^{\prime}}=x_{n}\left(\bmod \mathbb{Z}^{d}\right)$, since

$\overline{\mathcal{L}}_{\tau}\left(x_{m}, x_{m+1}, \ldots, x_{n}\right)=u\left(x_{n}\right)-u\left(x_{m}\right)=u\left(y_{n^{\prime}}\right)-u\left(y_{m^{\prime}}\right) \leq \overline{\mathcal{L}}_{\tau}\left(y_{m^{\prime}}, y_{m^{\prime}+1}, \ldots, y_{n^{\prime}}\right)$,

we obtain that $\left\{x_{k}\right\}_{k \in \mathbb{Z}}$ is a strongly minimizing configuration.

Notice that the existence of a $u$-calibrated configuration of a sub-action gives an equivalent definition of the holomic minimizing value $\bar{L}(\tau)$ as defined in 3.5

$$
\tau \bar{L}(\tau)=\inf _{\left\{x_{k}\right\} \in\left(\mathbb{R}^{d}\right)^{\mathbb{Z}}} \liminf _{n-m \rightarrow \infty} \frac{1}{n-m} \sum_{k=m}^{n-1} \mathcal{L}_{\tau}\left(x_{k}, x_{k+1}\right) .
$$

Furthermore, $u$-calibrated configurations are examples of critical configurations without assuming any ferromagnetic condition.

Lemma 5.7. Let $L(x, v)$ be a $C^{1}$ coercive Lagrangian. Any u-calibrated configuration of some $C^{0}$ periodic sub-action $u$ is critical.

Proof. Let $\left\{x_{k}\right\}_{k \in \mathbb{Z}}$ be a $u$-calibrated configuration. Lemma 5.6 implies $\left\{x_{k}\right\}_{k \in \mathbb{Z}}$ is minimizing and in particular satisfies

$$
\mathcal{L}_{\tau}\left(x_{k-1}, x_{k}, x_{k+1}\right) \leq \mathcal{L}_{\tau}\left(x_{k-1}, x, x_{k+1}\right), \quad \forall x \in \mathbb{R}^{d} .
$$

Therefore $\frac{\partial \mathcal{L}_{\tau}}{\partial y}\left(x_{k-1}, x_{k}\right)+\frac{\partial \mathcal{L}_{\tau}}{\partial x}\left(x_{k}, x_{k+1}\right)=0$ for all $k \in \mathbb{Z}$ and $\left\{x_{k}\right\}_{k \in \mathbb{Z}} \in \Gamma_{\tau}(L)$.

We have seen in remark 3.6 that any holonomic measure can be lifted to a shift-invariant probability measure in $\Sigma / \sim$ and that

$$
\tau \bar{L}(\tau)=\min _{\hat{\mu} \sigma-\text { invariant }} \int_{\Sigma / \sim} \mathcal{L}_{\tau}\left(x_{0}, x_{1}\right) d \hat{\mu}(\underline{x}) .
$$

We show in the following proposition how to lift some minimizing holonomic probability measures to $\left(\Gamma_{\tau}(L), \sigma\right)$ or equivalently to $\left(\mathbb{T}^{d} \times \mathbb{R}^{d}, \Phi_{\tau}\right)$.

Proposition 5.8. Let $L(x, v)$ be a $C^{1}$ ferromagnetic coercive Lagrangian, then the minimizing holonomic value of $L$ is given by

$$
\bar{L}(\tau)=\min \left\{\int L(x, v) d \mu(x, v): \mu \in \mathcal{P}_{\tau}\left(\mathbb{T}^{d} \times \mathbb{R}^{d}\right), \mu \Phi_{\tau} \text {-invariant }\right\} .
$$

Proof. We already remarked that any $\Phi_{\tau}$-invariant probability is holonomic, then

$$
\bar{L}(\tau) \leq \min \left\{\int L(x, v) d \mu(x, v): \mu \in \mathcal{P}_{\tau}\left(\mathbb{T}^{d} \times \mathbb{R}^{d}\right), \mu \Phi_{\left.\tau^{\text {-invariant }}\right\}} .\right.
$$

If $\left\{x_{k}\right\}_{k \in \mathbb{Z}}$ is a $u$-calibrated configuration for some $C^{0}$ periodic sub-action $u$, then $\left\{x_{k}\right\}_{k \in \mathbb{Z}} \in \Gamma_{\tau}(L)$ by lemma 5.7. Therefore, thanks to the conjugation between $\left(\Gamma_{\tau}(L), \sigma\right)$ and $\left(\mathbb{T}^{d} \times \mathbb{R}^{d}, \Phi_{\tau}\right)$, if $v_{k}:=\frac{x_{k+1}-x_{k}}{\tau}$, then $\left(x_{k}, v_{k}\right)=\Phi_{\tau}^{k}\left(x_{0}, v_{0}\right)$ for all 
$k \in \mathbb{Z}$ and $v_{k}$ is uniformly bounded by lemma 5.6. Let $\mu \in \mathcal{P}_{\tau}\left(\mathbb{T}^{d} \times \mathbb{R}^{d}\right)$ be a weak limit of some convergent subsequence

$$
\mu_{n_{l}}=\frac{1}{n_{l}} \sum_{k=0}^{n_{l}-1} \delta_{\Phi_{\tau}^{k}\left(x_{0}, v_{0}\right)} .
$$

Then $\mu$ is $\Phi_{\tau}$-invariant and we have

$$
\begin{aligned}
\int L(x, v) d \mu(x, v) & =\lim _{l \rightarrow \infty} \frac{1}{n_{l}} \sum_{k=0}^{n_{l}-1} L \circ \Phi_{\tau}^{k}\left(x_{0}, v_{0}\right) \\
& =\lim _{l \rightarrow \infty} \frac{1}{n_{l}}\left[\frac{u\left(x_{n_{l}}\right)-u\left(x_{0}\right)}{\tau}+n_{l} \bar{L}(\tau)\right]=\bar{L}(\tau) .
\end{aligned}
$$

We will see in the next section that, for ferromagnetic Lagrangians, all minimizing holonomic measures are actually $\Phi_{\tau}$-invariant.

\section{Graph property and Mather set}

In the setting of continuous-time periodic, strictly convex, superlinear and complete $C^{2}$-Lagrangians on a compact and connected $C^{\infty}$ manifold, J. N. Mather showed (see [51]) that measures invariant under the Euler-Lagrange flow which are action minimizing can be seen as Lipschitz sections of the tangent bundle. Our main goal in this section (see theorem 6.10) is to obtain a similar graph property.

Definition 6.1. Let $L(x, v)$ be a $C^{0}$ coercive Lagrangian. We call Mather set the set

$$
\mathcal{M}_{\tau}(L)=\operatorname{closure}\left(\bigcup\left\{\operatorname{supp}(\mu): \mu \in \mathcal{P}_{\tau}\left(\mathbb{T}^{d} \times \mathbb{R}^{d}\right) \text { and } \mu \text { is minimizing }\right\}\right),
$$

where $\operatorname{supp}(\mu)$ denotes the support of the probability $\mu$.

Proposition 3.7 implies that minimizing holonomic measures do exist, which shows that the Mather set is nonempty.

Definition 6.2. Let $L(x, v)$ be a $C^{0}$ coercive Lagrangian and $u$ be a $C^{0}$ periodic sub-action for $L$. We call nil locus of $u$ the set

$$
\mathcal{N}_{\tau}(L, u)=\left\{(x, v) \in \mathbb{T}^{d} \times \mathbb{R}^{d}: \tau L(x, v)=u(x+\tau v)-u(x)+\tau \bar{L}(\tau)\right\} .
$$

We observe that coerciveness guarantees all nil loci are nonempty. The following proposition shows that $\mathcal{N}_{\tau}(L, u)$ actually contains the support of any minimizing holonomic measure.

Proposition 6.3. Let $L(x, v)$ be a $C^{0}$ coercive Lagrangian. Then, for any subaction $u \in C^{0}\left(\mathbb{T}^{d}\right)$ with respect to $L$, we have $\mathcal{M}_{\tau}(L) \subset \mathcal{N}_{\tau}(L, u)$. 
Proof. Consider a minimizing holonomic measure $\mu \in \mathcal{P}_{\tau}\left(\mathbb{T}^{d} \times \mathbb{R}^{d}\right)$. Since one has both $\tau L(x, v)+u(x)-u(x+\tau v)-\tau \bar{L}(\tau) \geq 0$ and

$$
\int[\tau L(x, v)+u(x)-u(x+\tau v)-\tau \bar{L}(\tau)] d \mu(x, v)=0
$$

$\tau L(x, v)=u(x+\tau v)-u(x)+\tau \bar{L}(\tau)$ holds everywhere on the support of $\mu$.

As in the proof of lemma 5.6, the coerciveness assumption of $L$ implies that any nil locus is compact. More precisely, we have

Corollary 6.4. Let $L(x, v)$ be a $C^{0}$ coercive Lagrangian and $u \in C^{0}\left(\mathbb{T}^{d}\right)$ be a subaction with respect to $L$. If $(x, v) \in \mathcal{N}_{\tau}(L, u)$, then $\|v\|_{\infty} \leq R_{\tau}$. In particular, the support of any minimizing holonomic measure is compact.

Proof. If $\|v\|_{\infty}>R_{\tau}$, then $L(x, v)>L\left(x, v-\frac{1}{\tau}\lfloor\tau v\rfloor\right)$. Assume $(x, v) \in \mathcal{N}_{\tau}(L, u)$, then

$$
\begin{aligned}
u(x+\tau v)-u(x) & =\tau L(x, v)-\tau \bar{L}(\tau)>\tau L\left(x, v-\frac{1}{\tau}\lfloor\tau v\rfloor\right)-\tau \bar{L}(\tau) \\
& \geq u\left(x+\tau\left(v-\frac{1}{\tau}\lfloor\tau v\rfloor\right)\right)-u(x)=u(x+\tau v)-u(x) .
\end{aligned}
$$

We obtain a contradiction, therefore $\|v\|_{\infty} \leq R_{\tau}$.

We assume from now on in this section that $L$ is $C^{1}$ and coercive. We prove that any sub-action is continuously differentiable on the projected Mather set $p r^{1}\left(\mathcal{M}_{\tau}(L)\right)$, where $p r^{1}: \mathbb{T}^{d} \times \mathbb{R}^{d} \rightarrow \mathbb{T}^{d}$ denotes the first canonical projection. When $L$ is in addition ferromagnetic, we prove that $\mathcal{M}_{\tau}(L)$ is a graph over its projection into $\mathbb{T}^{d}$. Recall that $\Pi_{\tau}$ has been introduced in definition 2.6.

Lemma 6.5. Let $\mu$ be a holonomic measure with compact support, then for any $x \in \operatorname{pr}^{1}(\operatorname{supp}(\mu))$, there exists a configuration $\underline{x}:=\left\{x_{k}\right\}_{k \in \mathbb{Z}}$ in $\mathbb{R}^{d}$ such that $x_{0}=x$ and $\Pi_{\tau} \circ \sigma^{k}(\underline{x})=\left(x_{k}, \frac{x_{k+1}-x_{k}}{\tau}\right) \in \operatorname{supp}(\mu)$ for all $k \in \mathbb{Z}$.

Proof. From proposition 3.4, we naturally associate to $\mu$ a normalized invariant transshipment $\pi$ in $\mathbb{R}^{d} \times \mathbb{R}^{d}$. Let $p r^{1,2}: \mathbb{R}^{d} \times \mathbb{R}^{d} \rightarrow \mathbb{R}^{d}$ be the two canonical projections. Since $\mu$ has compact support, the support of $\pi$ has compact horizontal and vertical slices. Then $S^{1,2}:=p r^{1,2}(\operatorname{supp}(\pi))$ are closed sets. We always have $S^{1,2} \subseteq \operatorname{supp}\left(p r^{1,2}(\pi)\right)$. Since $S^{1,2}$ are closed, necessarily $S^{1,2}=\operatorname{supp}\left(p r_{*}^{1,2}(\pi)\right)$. Since $\pi$ is a transshipment, $p r_{*}^{1}(\pi)=p r_{*}^{2}(\pi)$ and $S^{1}=S^{2}$. Let $x_{0} \in S^{1}$, then there exists $x_{1}$ such that $\left(x_{0}, x_{1}\right) \in \operatorname{supp}(\pi)$. Since $x_{1} \in S^{2}=S^{1}$, there exists $x_{2}$ such that $\left(x_{1}, x_{2}\right) \in \operatorname{supp}(\pi)$, and so on. We thus obtain a forward and backward orbit $\left\{x_{k}\right\}_{k \in \mathbb{Z}}$ of points $\left(x_{k}, x_{k+1}\right)$ in the support of $\pi$ or equivalently an orbit $\left\{\left(x_{k}, v_{k}=\left(x_{k+1}-x_{k}\right) / \tau\right)\right\}_{k \in \mathbb{Z}}$ of points in the support of $\mu$.

In order to prove the differentiability of any sub-action on the projected Mather set, we introduce two intermediate notions of calibration. 
Definition 6.6. Let $u \in C^{0}\left(\mathbb{R}^{d}\right)$ be a $\mathbb{Z}^{d}$-periodic sub-action for L. A couple $\left(x_{0}, x_{1}\right) \in \mathbb{R}^{d} \times \mathbb{R}^{d}$ is called $u$-calibrated if $\mathcal{L}_{\tau}\left(x_{0}, x_{1}\right)=u\left(x_{1}\right)-u\left(x_{0}\right)+\tau \bar{L}(\tau)$. A triple $\left(x_{-1}, x_{0}, x_{1}\right)$ is called $u$-calibrated if both $\left(x_{-1}, x_{0}\right)$ and $\left(x_{0}, x_{1}\right)$ are $u$ calibrated.

Lemma 6.7. Let $L(x, v)$ be a $C^{1}$ coercive Lagrangian and $u \in C^{0}\left(\mathbb{T}^{d}\right)$ be a subaction. Then, for any $u$-calibrated couple $\left(x_{0}, x_{1}\right) \in \mathbb{R}^{d} \times \mathbb{R}^{d}$, we have

$$
\begin{gathered}
\limsup _{\|h\|_{\infty} \rightarrow 0} \frac{1}{\|h\|_{\infty}}\left[u\left(x_{1}+h\right)-u\left(x_{1}\right)-\left\langle\frac{\partial \mathcal{L}_{\tau}}{\partial y}\left(x_{0}, x_{1}\right), h\right\rangle\right] \leq 0 \quad \text { and } \\
\liminf _{\|h\|_{\infty} \rightarrow 0} \frac{1}{\|h\|_{\infty}}\left[u\left(x_{0}+h\right)-u\left(x_{0}\right)-\left\langle-\frac{\partial \mathcal{L}_{\tau}}{\partial x}\left(x_{0}, x_{1}\right), h\right\rangle\right] \geq 0 .
\end{gathered}
$$

Proof. Indeed, since $u$ is a sub-action, we have on the one hand

$$
\begin{gathered}
u\left(x_{1}+h\right) \leq u\left(x_{0}\right)+\mathcal{L}_{\tau}\left(x_{0}, x_{1}+h\right)-\tau \bar{L}(\tau) \quad \text { and } \\
u\left(x_{1}\right) \leq u\left(x_{0}+h\right)+\mathcal{L}_{\tau}\left(x_{0}+h, x_{1}\right)-\tau \bar{L}(\tau), \quad \forall h \in \mathbb{R}^{d} .
\end{gathered}
$$

On the other hand, $u\left(x_{1}\right)=u\left(x_{0}\right)+\mathcal{L}_{\tau}\left(x_{0}, x_{1}\right)-\tau \bar{L}(\tau)$, which implies

$$
\begin{gathered}
u\left(x_{1}+h\right)-u\left(x_{1}\right) \leq\left[\mathcal{L}_{\tau}\left(x_{0}, x_{1}+h\right)-\mathcal{L}_{\tau}\left(x_{0}, x_{1}\right)\right] \text { and } \\
u\left(x_{0}+h\right)-u\left(x_{0}\right) \geq\left[\mathcal{L}_{\tau}\left(x_{0}, x_{1}\right)-\mathcal{L}_{\tau}\left(x_{0}+h, x_{1}\right)\right] .
\end{gathered}
$$

The lemma follows from the differentiability of $L$.

Although we could use the theory of subdifferentiability to derive the next lemma, for the sake of completeness, we prefer to give a direct proof.

Lemma 6.8. Let $L(x, v)$ be a $C^{1}$ coercive Lagrangian and $u \in C^{0}\left(\mathbb{T}^{d}\right)$ be a subaction. Let $\mathcal{K}_{\tau}(L, u)$ denote the set of mid-points $x_{0}$ of all u-calibrated triples $\left(x_{-1}, x_{0}, x_{1}\right)$.

i. If $\left(x_{-1}, x_{0}, x_{1}\right)$ is $u$-calibrated, then $u$ is differentiable at $x_{0}$ and

$$
\begin{aligned}
D u\left(x_{0}\right) & =\frac{\partial \mathcal{L}_{\tau}}{\partial y}\left(x_{-1}, x_{0}\right)=-\frac{\partial \mathcal{L}_{\tau}}{\partial x}\left(x_{0}, x_{1}\right) \\
& =\frac{\partial L}{\partial v}\left(x_{-1}, \frac{x_{0}-x_{-1}}{\tau}\right)=\frac{\partial L}{\partial v}\left(x_{0}, \frac{x_{1}-x_{0}}{\tau}\right)-\tau \frac{\partial L}{\partial x}\left(x_{0}, \frac{x_{1}-x_{0}}{\tau}\right) .
\end{aligned}
$$

ii. The map $D u: \mathcal{K}_{\tau}(L, u) \rightarrow \mathbb{R}^{d}$ is uniformly continuous independently of $u$.

iii. If $L$ is in addition ferromagnetic, then there exists at most one u-calibrated configuration passing through any $x_{0} \in \mathbb{R}^{d}$.

Proof. Item $i$. On the one hand, a $u$-calibrated triple is critical as in definition 2.3. Let $\nabla$ be the common derivative

$$
\nabla:=\frac{\partial \mathcal{L}_{\tau}}{\partial y}\left(x_{-1}, x_{0}\right)=-\frac{\partial \mathcal{L}_{\tau}}{\partial x}\left(x_{0}, x_{1}\right)
$$


On the other hand, lemma 6.7 implies that

$$
\limsup _{\|h\|_{\infty} \rightarrow 0} \frac{u\left(x_{0}+h\right)-u\left(x_{0}\right)-\langle\nabla, h\rangle}{\|h\|_{\infty}} \leq 0 \leq \liminf _{\|h\|_{\infty} \rightarrow 0} \frac{u\left(x_{0}+h\right)-u\left(x_{0}\right)-\langle\nabla, h\rangle}{\|h\|_{\infty}},
$$

which shows that $D u\left(x_{0}\right)=\nabla$.

Item ii. We begin by showing that there exists a positive function $C_{\tau}(h)$ defined for all $h \in \mathbb{R}^{d}$, depending only on $\tau$ and $L$, such that $C_{\tau}(h) \rightarrow 0$ when $h \rightarrow 0$ and

$$
\left|u\left(x_{0}+h\right)-u\left(x_{0}\right)-\left\langle D u\left(x_{0}\right), h\right\rangle\right| \leq C_{\tau}(h)\|h\|_{\infty},
$$

for all $h \in \mathbb{R}^{d}$, all $x_{0} \in \mathcal{K}_{\tau}(L, u)$ and all sub-action $u$. Let $\left(x_{-1}, x_{0}, x_{1}\right)$ be a $u$-calibrated triple. On the one hand,

$$
u\left(x_{1}\right)-u\left(x_{0}\right)-\mathcal{L}_{\tau}\left(x_{0}, x_{1}\right)=\tau \bar{L}(\tau) \geq u\left(x_{1}\right)-u\left(x_{0}+h\right)-\mathcal{L}_{\tau}\left(x_{0}+h, x_{1}\right),
$$

and by eliminating $u\left(x_{1}\right)$ one obtain

$u\left(x_{0}+h\right)-u\left(x_{0}\right)-\left\langle D u\left(x_{0}\right), h\right\rangle \geq-\left[\mathcal{L}_{\tau}\left(x_{0}+h, x_{1}\right)-\mathcal{L}_{\tau}\left(x_{0}, x_{1}\right)-\left\langle\frac{\partial \mathcal{L}_{\tau}}{\partial x}\left(x_{0}, x_{1}\right) . h\right\rangle\right]$.

On the other hand,

$$
u\left(x_{0}\right)-u\left(x_{-1}\right)-\mathcal{L}_{\tau}\left(x_{-1}, x_{0}\right)=-\tau \bar{L}(\tau) \geq u\left(x_{0}+h\right)-u\left(x_{-1}\right)-\mathcal{L}_{\tau}\left(x_{-1}, x_{0}+h\right),
$$

and by eliminating $u\left(x_{-1}\right)$ one obtain

$u\left(x_{0}+h\right)-u\left(x_{0}\right)-\left\langle D u\left(x_{0}\right), h\right\rangle \leq \mathcal{L}_{\tau}\left(x_{-1}, x_{0}+h\right)-\mathcal{L}_{\tau}\left(x_{-1}, x_{0}\right)-\left\langle\frac{\partial \mathcal{L}_{\tau}}{\partial y}\left(x_{-1}, x_{0}\right), h\right\rangle$.

Notice that $\left\|x_{0}-x_{-1}\right\|_{\infty},\left\|x_{1}-x_{0}\right\|_{\infty} \leq \tau R(\tau)$ whenever $\left(x_{-1}, x_{0}, x_{1}\right) \in \mathcal{K}_{\tau}(L, u)$ and that $\mathcal{L}_{\tau}(x, y)$ is invariant by the diagonal $\mathbb{Z}^{d}$-translation. Define

$$
\begin{aligned}
C_{\tau}^{\prime}(h) & :=\max _{\left\|x_{1}-x_{0}\right\|_{\infty} \leq \tau R(\tau)} \max _{s \in[0,1]}\left\|\frac{\partial \mathcal{L}_{\tau}}{\partial x}\left(x_{0}+s h, x_{1}\right)-\frac{\partial \mathcal{L}_{\tau}}{\partial x}\left(x_{0}, x_{1}\right)\right\|_{\infty} \\
C_{\tau}^{\prime \prime}(h) & :=\max _{\left\|x_{0}-x_{-1}\right\|_{\infty} \leq \tau R(\tau)} \max _{s \in[0,1]}\left\|\frac{\partial \mathcal{L}_{\tau}}{\partial y}\left(x_{-1}, x_{0}+s h\right)-\frac{\partial \mathcal{L}_{\tau}}{\partial y}\left(x_{-1}, x_{0}\right)\right\|_{\infty} .
\end{aligned}
$$

Then $C_{\tau}(h)=\max \left(C_{\tau}^{\prime}(h), C_{\tau}^{\prime \prime}(h)\right)$ is the desired function.

We now show that $D u\left(x_{0}\right)$ is uniformly continuous on $\mathcal{K}_{\tau}(L, u)$. Notice that

$$
\left|u\left(x_{0}\right)-u\left(x_{0}-h\right)-\left\langle D u\left(x_{0}\right), h\right\rangle\right| \leq C_{\tau}(-h)\|h\|_{\infty}, \quad \forall h \in \mathbb{R}^{d} .
$$

Let $x_{0}$ and $x_{0}^{\prime}$ be two distinct mid-points of $\mathcal{K}_{\tau}(L, u)$. Then, for any $h$,

$$
\begin{gathered}
\left|u\left(x_{0}+h\right)-u\left(x_{0}\right)-\left\langle D u\left(x_{0}\right), h\right\rangle\right| \leq C_{\tau}(h)\|h\|_{\infty}, \\
\left|u\left(x_{0}^{\prime}\right)-u\left(x_{0}+h\right)-\left\langle D u\left(x_{0}^{\prime}\right), x_{0}^{\prime}-x_{0}-h\right\rangle\right| \leq C_{\tau}\left(x_{0}+h-x_{0}^{\prime}\right)\left\|x_{0}+h-x_{0}^{\prime}\right\|_{\infty}, \\
\left|u\left(x_{0}\right)-u\left(x_{0}^{\prime}\right)-\left\langle D u\left(x_{0}^{\prime}\right), x_{0}-x_{0}^{\prime}\right\rangle\right| \leq C_{\tau}\left(x_{0}-x_{0}^{\prime}\right)\left\|x_{0}-x_{0}^{\prime}\right\|_{\infty} .
\end{gathered}
$$


By adding the three inequalities and by taking any $\|h\|_{\infty}=\left\|x_{0}-x_{0}^{\prime}\right\|_{\infty}$, we obtain

$$
\left|\left\langle D u\left(x_{0}^{\prime}\right)-D u\left(x_{0}\right), h\right\rangle\right| \leq C_{\tau}\left(x_{0}, x_{0}^{\prime}\right)\|h\|_{\infty},
$$

where $C_{\tau}\left(x_{0}, x_{0}^{\prime}\right):=\sup _{\|h\|_{\infty}=\left\|x_{0}-x_{0}^{\prime}\right\|_{\infty}}\left[C_{\tau}(h)+2 C_{\tau}\left(x_{0}+h-x_{0}^{\prime}\right)+C_{\tau}\left(x_{0}-x_{0}^{\prime}\right)\right]$. Therefore $\left\|D u\left(x_{0}\right)-D u\left(x_{0}^{\prime}\right)\right\|_{\infty} \leq C_{\tau}\left(x_{0}, x_{0}^{\prime}\right)$ and $D u\left(x_{0}\right)$ is uniformly continuous.

Item iii. If $\left\{x_{k}\right\}_{k \in \mathbb{Z}}$ is a $u$-calibrated configuration, then $D u\left(x_{k}\right)$ exists for all $k$ and the two equations $D u\left(x_{k}\right)=\frac{\partial \mathcal{L}_{\tau}}{\partial y}\left(x_{k-1}, x_{k}\right)$ and $D u\left(x_{k}\right)=-\frac{\partial \mathcal{L}_{\tau}}{\partial x}\left(x_{k}, x_{k+1}\right)$ shows that $x_{k-1}$ and $x_{k+1}$ are known as soon as $x_{k}$ is known and $L$ is ferromagnetic.

The following proposition is now a direct consequence of proposition 6.3 and lemmas 6.5 and 6.8 .

Proposition 6.9. Let $L(x, v)$ be a $C^{1}$ coercive Lagrangian. Then any sub-action $u \in C^{0}\left(\mathbb{T}^{d}\right)$ with respect to $L$ is continuously differentiable on the projected Mather set $\mathrm{pr}^{1}\left(\mathcal{M}_{\tau}(L)\right)$, where $\mathrm{pr}^{1}: \mathbb{T}^{d} \times \mathbb{R}^{d} \rightarrow \mathbb{T}^{d}$ denotes the first canonical projection. If $L$ is in addition $C^{1,1}$, then $D u: p r^{1}\left(\mathcal{M}_{\tau}(L)\right) \rightarrow \mathbb{R}$ is Lipschitz uniformly in $u$.

Proof. Recall from lemma 6.8 that $\mathcal{K}_{\tau}(L, u)$ denotes the set of mid-points of $u$ calibrated triples. From lemmas 6.5 and proposition 6.3 , we deduce that

$$
p r^{1}\left(\mathcal{M}_{\tau}(L)\right) \subseteq \mathcal{K}_{\tau}(L, u)
$$

From 6.8 , we obtain that $D u: \mathbb{T}^{d} \rightarrow \mathbb{R}^{d}$ is continuous.

Mather graph property is then an easy consequence of the previous study in the case of ferromagnetic Lagrangians.

Theorem 6.10. Let $L(x, v)$ be a $C^{1}$ ferromagnetic coercive Lagrangian. Then there exists a continuous map

$$
V_{\tau}: p r^{1}\left(\mathcal{M}_{\tau}(L)\right) \rightarrow \mathbb{R}^{d}, \quad\left\|V_{\tau}\right\|_{\infty} \leq R_{\tau},
$$

such that $\mathcal{M}_{\tau}(L)$ is a graph over its projection, that is,

$$
\mathcal{M}_{\tau}(L)=\operatorname{graph}\left(V_{\tau}\right)=\left\{\left(x, V_{\tau}(x)\right) \mid x \in p r^{1}\left(\mathcal{M}_{\tau}(L)\right\} .\right.
$$

Moreover, $\mathcal{M}_{\tau}(L)$ is compact and $\Phi_{\tau}$-invariant, any minimizing holonomic probability measure $\mu$ is $\Phi_{\tau}$-invariant and, for any sub-action $u \in C^{0}\left(\mathbb{T}^{d}\right)$, one has

$$
D u(x)=\frac{\partial L}{\partial v}\left(x, V_{\tau}(x)\right)-\tau \frac{\partial L}{\partial x}\left(x, V_{\tau}(x)\right), \quad \forall x \in p r^{1}\left(\mathcal{M}_{\tau}(L)\right) .
$$

Proof. Let $u \in C^{0}\left(\mathbb{T}^{d}\right)$ be any sub-action. From lemma 6.8 , we know that $D u(x)$ exists and is continuous for all $x \in \mathcal{K}_{\tau}(L, u)$. Since $L$ is ferromagnetic, we define uniquely $V_{\tau}(x)$ by the following implicit equation

$$
D u(x)=\frac{\partial L}{\partial v}\left(x, V_{\tau}(x)\right)-\tau \frac{\partial L}{\partial x}\left(x, V_{\tau}(x)\right), \quad \forall x \in \mathcal{K}_{\tau}(L, u) .
$$

Then $V_{\tau}$ becomes continuous on $\mathcal{K}_{\tau}(L, u)$. 
Assume now that $x \in \operatorname{pr}^{1}\left(\mathcal{M}_{\tau}(L)\right)$. Consider a point $x_{-1} \in \mathbb{T}^{d}$ with $\left(x_{-1}, x\right)$ $u$-calibrated. Take $v \in \mathbb{R}^{d}$ such that $(x, v) \in \mathcal{M}_{\tau}(L)$ and define $x_{1}=x+\tau v$. Then $\left(x_{-1}, x, x_{1}\right)$ is $u$-calibrated and, thanks to lemma 6.8 , we have

$$
D u(x)=\frac{\partial L}{\partial v}(x, v)-\tau \frac{\partial L}{\partial x}(x, v) .
$$

Necessarily $v=V_{\tau}(x),\left\|V_{\tau}(x)\right\|_{\infty} \leq R_{\tau}$ and $V_{\tau}(x)$ is independent of the choice of $u$. From lemma 6.5, we know there exist $u$-calibrated triples passing through $x$ consisting of points of $\operatorname{pr}^{1}\left(\mathcal{M}_{\tau}(L)\right)$. From the ferromagnetic property, we deduce that this triple is unique. Thus $x_{1} \in \operatorname{pr}^{1}\left(\mathcal{M}_{\tau}(L)\right) \subseteq \mathcal{K}_{\tau}(L, u)$ and

$$
D u\left(x_{1}\right)=\frac{\partial L}{\partial v}\left(x, V_{\tau}(x)\right)=\frac{\partial L}{\partial v}\left(x_{1}, V_{\tau}\left(x_{1}\right)\right)-\tau \frac{\partial L}{\partial x}\left(x_{1}, V_{\tau}\left(x_{1}\right)\right) .
$$

From the definition of $\Phi_{\tau}$ (see definition 2.5), we obtain

$$
\Phi_{\tau}(x, v)=\left(x+\tau v, V_{\tau}(x+\tau v)\right), \quad \forall(x, v) \in \mathcal{M}_{\tau}(L) .
$$

In particular, $\Phi_{\tau}$ preserves the Mather set (the reverse inclusion is proved similarly)

$$
\Phi_{\tau}\left(\mathcal{M}_{\tau}(L)\right)=\mathcal{M}_{\tau}(L)
$$

Let $\mu \in \mathcal{P}_{\tau}\left(\mathbb{T}^{d} \times \mathbb{R}^{d}\right)$ be a minimizing holonomic probability measure. For any bounded Borel function $\varphi: \mathbb{T}^{d} \times \mathbb{R}^{d} \rightarrow \mathbb{R}$, from the previous identity, we have

$$
\begin{aligned}
\int \varphi \circ \Phi_{\tau}(x, v) d \mu(x, v) & =\int_{\mathcal{M}_{\tau}(L)} \varphi\left(x+\tau v, V_{\tau}(x+\tau v)\right) d \mu(x, v) \\
& =\int_{\mathcal{M}_{\tau}(L)} \varphi\left(x, V_{\tau}(x)\right) d \mu(x, v)=\int \varphi(x, v) d \mu(x, v),
\end{aligned}
$$

which means the $\Phi_{\tau}$-invariance of the measure $\mu$.

The last statement of theorem 6.10 is similar to a known result in the case of Lagrangian theory. For a continuous-time periodic, strictly convex, superlinear and complete $C^{\infty}$-Lagrangian on a closed Riemannian manifold, R. Mañé showed (see proposition 1.3 of [44]) that any minimizing holonomic measure is invariant under the Euler-Lagrange equations.

\section{The Aubry set}

In Aubry-Mather theory for continuous-time Lagrangian dynamics, there are generally two strategies for introducing the Aubry set: A. Fathi's formulation (see [23]) using the notion of conjugate weak KAM solutions and G. Contreras and R. Iturriaga construction (see [17]) using the notion of static curves. Both approaches request intrinsically a differentiable Lagrangian.

We have chosen a different approach which is closer to a more usual definition in ergodic optimization theory and which has the main advantage of requiring only $C^{0}$ smoothness. The Aubry set will be introduced using the following concept. 
Definition 7.1. We call periodic configuration of type $(q, p)$ a finite configuration $\left(x_{0}, x_{1}, \cdots, x_{q}\right)$ of points of $\mathbb{R}^{d}$ such that $x_{q}=x_{0}+p, q \geq 1$ and $p \in \mathbb{Z}^{d}$. Such a finite configuration determines uniquely a bi-infinite configuration $\left\{x_{k}\right\}_{k \in \mathbb{Z}}$ satisfying $x_{q+k}=x_{k}+p$ for all $k \in \mathbb{Z}$.

The notion of Aubry point below is similar to the one of non-wandering point with respect a potential used in ergodic optimization (see, for instance, [18, 31, 32]). A similar projected Aubry set in [35] has also been used in the discrete AubryMather problem. Recall that $\overline{\mathcal{L}}_{\tau}\left(x_{0}, x_{1}, \cdots, x_{q}\right)=\sum_{k=0}^{q-1}\left[\mathcal{L}_{\tau}\left(x_{k}, x_{k+1}\right)-\tau \bar{L}(\tau)\right]$.

Definition 7.2. Let $L(x, v): \mathbb{T}^{d} \times \mathbb{R}^{d} \rightarrow \mathbb{R}$ be a $C^{0}$ coercive Lagrangian. A point $(x, v) \in \mathbb{T}^{d} \times \mathbb{R}^{d}$ is said to be an Aubry point if, for any $\epsilon>0$, there exists a periodic configuration of type $(q, p),\left(x_{0}, x_{1}, \cdots, x_{q}\right)$, such that

$$
\left\|x-x_{0}\right\|_{\infty}<\epsilon, \quad\left\|x+\tau v-x_{1}\right\|_{\infty}<\epsilon \quad \text { and } \quad\left|\overline{\mathcal{L}}_{\tau}\left(x_{0}, x_{1}, \cdots, x_{q}\right)\right| \leq \epsilon .
$$

The Aubry set $\mathcal{A}_{\tau}(L)$ is by definiton the set of all Aubry points.

Notice that the Aubry set depends on $L$ modulo any coboundary, that is, for all function $\psi \in C^{0}\left(\mathbb{T}^{d}\right)$ and any constant $c \in \mathbb{R}, \mathcal{A}_{\tau}(L)=\mathcal{A}_{\tau}\left(L-\Delta_{\tau} \psi-c\right)$, where $\Delta_{\tau} \psi(x, v):=\psi(x+\tau v)-\psi(x)$. Notice also that $\overline{\mathcal{L}}_{\tau}\left(x_{0}, x_{1}, \cdots, x_{q}\right) \geq 0$ for any periodic configuration of type $(q, p)$, since $\overline{\mathcal{L}}_{\tau}\left(x_{0}, x_{1}, \cdots, x_{q}\right)$ is unchanged if, instead of $L$, we use $L-\frac{1}{\tau} \Delta_{\tau} u-\bar{L}(\tau) \geq 0$ for some sub-action $u$.

It is easy to see that the Aubry set is a closed subset of $\mathbb{T}^{d} \times \mathbb{R}^{d}$. The fact that it is a non empty set is proved in the following proposition.

Proposition 7.3. Let $L(x, v)$ be a $C^{0}$ coercive Lagrangian and $u \in C^{0}\left(\mathbb{T}^{d}\right)$ be a sub-action with respect to $L$. Then $\mathcal{M}_{\tau}(L) \subset \mathcal{A}_{\tau}(L) \subset \mathcal{N}_{\tau}(L, u)$.

Proof. We begin by proving the second inclusion. Define the associated normalized Lagrangian $E(x, v):=L(x, v)-\frac{1}{\tau}[u(x+\tau v)-u(x)]-\bar{L}(\tau)$ and the corresponding interaction energy $\mathcal{E}_{\tau}(x, y)$. Then $E(x, v) \geq 0$ for all $(x, v) \in \mathbb{T}^{d} \times \mathbb{R}^{d}$ and $\bar{E}(\tau)=0$. For any $\epsilon>0$, there exists a periodic configuration $\left(x_{0}, x_{1}, \cdots, x_{q}\right)$ such that

$$
0 \leq \overline{\mathcal{E}}_{\tau}\left(x_{0}, x_{1}\right) \leq \overline{\mathcal{E}}_{\tau}\left(x_{0}, x_{1}, \cdots, x_{q}\right)=\overline{\mathcal{L}}_{\tau}\left(x_{0}, x_{1}, \cdots, x_{q}\right) \leq \epsilon .
$$

Letting $\epsilon$ go to 0 , we obtain $\mathcal{E}_{\tau}(x, x+\tau v)=0$ or $(x, v) \in \mathcal{N}_{\tau}(L, u)$.

We now prove the first inclusion. The proof of this part is non trivial and requires the use of Atkinson's theorem which we recall in 7.4. Let $\mu$ be a minimizing holonomic probability measure and $(x, v) \in \operatorname{supp}(\mu)$. By proposition 3.4, the measure $\mu$ can be lifted to a normalized shift-invariant Markov chain $\hat{\mu}$ on $\Sigma / \sim$. Remark 3.6 tells us that $\hat{\mu}$ is a minimizing shift-invariant probability in following sense

$$
\tau \bar{L}(\tau)=\int_{\Sigma / \sim} \mathcal{L}_{\tau}\left(x_{0}, x_{1}\right) d \hat{\mu}(\underline{x})=\inf _{\hat{\nu}} \int_{\sigma \text {-invariant }} \mathcal{L}_{\Sigma / \sim}\left(x_{0}, x_{1}\right) d \hat{\nu}(\underline{x}) .
$$

Take $\epsilon>0$. Let $B_{\epsilon}$ denote an open ball of radius $\eta(\epsilon) \in(0, \epsilon)$ around the point $(x, v)$ such that the oscilation of $\mathcal{L}_{\tau}\left(x_{0}, x_{1}\right)$ on $\hat{B}_{\epsilon}:=\Pi_{\tau}^{-1}\left(B_{\epsilon}\right)$ is less than $\epsilon$. Then 
$\hat{\mu}\left(\hat{B}_{\epsilon}\right)=\mu\left(B_{\epsilon}\right)>0$ and, by the ergodic decomposition theorem (see, for instance, chapter 7 of [36]), there exists an ergodic minimizing shift-invariant probability $\hat{\nu}$ which satisfies

$$
\hat{\nu}\left(\hat{B}_{\epsilon}\right)>0 \quad \text { and } \quad \tau \bar{L}(\tau)=\int_{\Sigma / \sim} \mathcal{L}_{\tau}\left(x_{0}, x_{1}\right) d \hat{\nu}(\underline{x}) .
$$

Atkinson's theorem implies that there exist a point $\underline{x}=\left\{x_{k}\right\}_{k \in \mathbb{Z}} \in \hat{B}_{\epsilon}$ and infinitely many positive integers $q$ such that $\sigma^{q}(\underline{x}) \in \hat{B}_{\epsilon}$ and $\left|\overline{\mathcal{L}}_{\tau}\left(x_{0}, x_{1}, \cdots, x_{q}\right)\right|<\epsilon$. By definition of $\hat{B}_{\epsilon}$, we may assume $x_{0}$ and $x_{1}$ close to $x$ and $x+\tau v$ within $\eta(\epsilon)$. Moreover, $x_{q}$ is close to $x_{0}+p$ within $\eta(\epsilon)$ for some $p \in \mathbb{Z}^{d}$. We have obtained a periodic configuration $\left(x_{q}-p, x_{1}, \cdots, x_{q}\right)$ beginning close to $(x, x+\tau v)$ and satisfying $\left|\overline{\mathcal{L}}_{\tau}\left(x_{q}-p, x_{1}, \cdots, x_{q}\right)\right|<2 \epsilon$. We have shown that $(x, v) \in \mathcal{A}_{\tau}(L)$.

Atkinson's theorem is well known. We recall the statement for completeness.

Theorem 7.4. (Atkinson's theorem [2]) Let $(Z, \mathfrak{C}, \lambda)$ be a probability space, $T: Z \rightarrow Z$ an ergodic measure preserving map, $f: Z \rightarrow \mathbb{R}$ an integrable function, $f \in L^{1}(\lambda)$, and $D \in \mathfrak{C}$ a measurable set of positive measure, $\lambda(D)>0$. Denote

$$
\begin{aligned}
& \Xi(f, D):=\{z \in D: \forall \epsilon>0, \exists n \geq 1 \text { with } \\
& \left.\qquad T^{n}(z) \in D \text { and }\left|\sum_{k=0}^{n-1} f \circ T^{k}(z)-n \int_{Z} f d \lambda\right|<\epsilon\right\} .
\end{aligned}
$$

Then $\lambda(\Xi(f, D))=\lambda(D)$.

We want now to prove that any sub-action is continuously differentiable on the Aubry set. We first show that a finite configuration with bounded interaction energy has bounded jumps independently of the length of the configuration.

Lemma 7.5. Let $L(x, v)$ be a $C^{0}$ coercive Lagrangian. Then for any $E>0$ there exists $R_{E}>0$ such that, for any $n \geq 1$ and any finite configuration $\left(x_{0}, x_{1}, \cdots, x_{n}\right)$ of length $n$ with interaction energy bounded from above by $E$,

$$
\overline{\mathcal{L}}_{\tau}\left(x_{0}, x_{1}, \cdots, x_{n}\right) \leq E \quad \Longrightarrow \quad\left\|x_{k}-x_{k-1}\right\|_{\infty} \leq R_{E}, \quad \forall k=1, \cdots, n .
$$

Proof. Let $u$ be a fixed $C^{0}\left(\mathbb{T}^{d}\right)$ sub-action. By coerciveness of $L$, for every $E>0$ there exists $R_{E}>0$ such that $\left|\overline{\mathcal{L}}_{\tau}(x, y)\right| \leq E+4\|u\|_{0} \Rightarrow\|y-x\|_{\infty}<R_{E}$. Then

$$
\begin{aligned}
0 \leq \overline{\mathcal{L}}_{\tau}\left(x_{k-1}, x_{k}\right)+u\left(x_{k-1}\right) & -u\left(x_{k}\right) \leq \\
& \leq \overline{\mathcal{L}}_{\tau}\left(x_{0}, x_{1}, \cdots, x_{n}\right)+u\left(x_{0}\right)-u\left(x_{n}\right) \leq E+2\|u\|_{0},
\end{aligned}
$$

$\left|\overline{\mathcal{L}}_{\tau}\left(x_{k-1}, x_{k}\right)\right| \leq E+4\|u\|_{0}$ and $\left\|x_{k}-x_{k-1}\right\|<R_{E}$.

We can now extend the conclusion of proposition 6.9.

Proposition 7.6. Let $u \in C^{0}\left(\mathbb{T}^{d}\right)$ be a sub-action with respect to a $C^{1}$ coercive Lagrangian $L(x, v)$. Then $u$ is continuously differentiable on the projected Aubry set $\operatorname{pr}^{1}\left(\mathcal{A}_{\tau}(L)\right)$. If $L$ is in addition $C^{1,1}$ then $D u: \operatorname{pr}^{1}\left(\mathcal{A}_{\tau}(L)\right) \rightarrow \mathbb{R}$ is Lipschitz uniformly in $u$. 
Proof. As in the proof of proposition 6.9, we just need to prove that

$$
\operatorname{pr}^{1}\left(\mathcal{A}_{\tau}(L) \subseteq \mathcal{K}_{\tau}(L, u)\right.
$$

We normalize again by defining

$$
\overline{\mathcal{E}}_{\tau}\left(x_{0}, \cdots, x_{n}\right)=\overline{\mathcal{L}}_{\tau}\left(x_{0}, \cdots, x_{n}\right)+u\left(x_{0}\right)-u\left(x_{n}\right) \geq 0 .
$$

Let $(x, v) \in \mathcal{A}_{\tau}(L), x_{0}=x$ and $x_{1}=x+\tau v$. Then there exist a sequence of periodic configurations $\left(x_{0}^{l}, x_{1}^{l}, \cdots, x_{q(l)}^{l}\right)$ and a sequence of integers $p^{l} \in \mathbb{Z}^{d}$ such that

$$
x_{0}^{l} \rightarrow x_{0}, \quad x_{1}^{l} \rightarrow x_{1}, \quad x_{q(l)}^{l}=x_{0}^{l}+p^{l} \quad \text { and } \quad \bar{\varepsilon}_{\tau}\left(x_{0}^{l}, x_{1}^{l}, \cdots, x_{q(l)}^{l}\right) \rightarrow 0 .
$$

From lemma 7.5 we obtain that $\left\{x_{q(l)}^{l}-x_{q(l)-1}^{l}\right\}_{l}$ is uniformly bounded. One can extract a converging subsequence of $\left\{x_{q(l)-1}^{l}-p^{l}\right\}_{l}$ to some $x_{-1} \in \mathbb{R}^{d}$. Since

$$
0 \leq \bar{\varepsilon}_{\tau}\left(x_{q(l)-1}^{l}-p^{l}, x_{q(l)}^{l}-p^{l}\right) \leq \bar{\varepsilon}_{\tau}\left(x_{0}^{l}, x_{1}^{l}, \cdots, x_{q(l)}^{l}\right),
$$

$\overline{\mathcal{E}}_{\tau}\left(x_{-1}, x_{0}\right)=0$ and $\left(x_{-1}, x_{0}, x_{1}\right)$ is a $u$-calibrated triple: $x \in \mathcal{K}_{\tau}(L, u)$.

We can now improve theorem 6.10 in the ferromagnetic case. As in definition 2.5, we recall that $\left(\mathbb{T}^{d} \times \mathbb{R}^{d}, \Phi_{\tau}\right)$ denotes the discrete Euler Lagrange map.

Theorem 7.7. Let $L(x, v)$ be a $C^{1}$ ferromagnetic coercive Lagrangian. Then $\mathcal{A}_{\tau}(L)$ is compact, $\Phi_{\tau}$-invariant and equal to the graph of some continuous map $V_{\tau}: p r^{1}\left(\mathcal{A}_{\tau}(L)\right) \rightarrow \mathbb{R}^{d}$.

Proof. The proof is similar to the proof of theorem 6.10 thanks to the fact that $\operatorname{pr}^{1}\left(\mathcal{A}_{\tau}(L)\right) \subseteq \mathcal{K}_{\tau}(L, u)$ for any continuous and periodic sub-action $u$ and to the fact that any $x \in \operatorname{pr}^{1}\left(\mathcal{A}_{\tau}(L)\right)$ is the projection of a configuration $\underline{x}=\left\{x_{k}\right\}_{k \in \mathbb{Z}}$ satisfying $\Pi_{\tau}\left(\sigma^{k}(\underline{x})\right) \in \mathcal{A}_{\tau}(L)$ for all $k \in \mathbb{Z}$. This is similar to lemma 6.5. The proof of this fact is given in the following lemma 7.8.

Lemma 7.8. Let $L(x, v)$ be a $C^{0}$ coercive Lagrangian. For any $(x, v) \in \mathcal{A}_{\tau}(L)$ there exists a configuration $\underline{x}=\left\{x_{k}\right\}_{k \in \mathbb{Z}}$ of points of $\mathbb{R}^{d}$ such that $\Pi_{\tau}\left(x_{0}, x_{1}\right)=(x, v)$ and $\Pi_{\tau}\left(\sigma^{k}(\underline{x})\right) \in \mathcal{A}_{\tau}(L)$ for all $k \in \mathbb{Z}$.

Proof. We begin by normalizing $L$ by assuming $L(x, v) \geq 0$ and $\bar{L}=0$. Let $(x, v) \in \mathcal{A}_{\tau}(L), x_{0}=x$ and $x_{1}=x_{0}+\tau v$. Then there exists a sequence of periodic configurations $\underline{x}^{l}=\left(x_{0}^{l}, x_{1}^{l}, \cdots, x_{q(l)}^{l}\right), x_{q(l)}^{l}=x_{0}^{l}+p^{l}$ for some $p^{l} \in \mathbb{Z}^{d}$ such that

$$
x_{0}^{l} \rightarrow x_{0}, \quad x_{1}^{l} \rightarrow x_{1} \quad \text { and } \quad 0 \leq \overline{\mathcal{L}}_{\tau}\left(x_{0}^{l}, x_{1}^{l}, \cdots, x_{q(l)}^{l}\right) \rightarrow 0 .
$$

From lemma 7.5 we know there exists $R>0$ such that all jumps are bounded uniformly, $\left\|x_{k}^{l}-x_{k+1}^{l}\right\|_{\infty}<R$ for all $k \in \mathbb{Z}$. By a diagonal procedure of extraction, there exists a subsequence of $\left\{\underline{x}^{l}\right\}_{l}$, that we call again $\left\{\underline{x}^{l}\right\}_{l}$, such that, for all $k \geq 0$, when $l \rightarrow \infty$ one has $x_{k}^{l} \rightarrow x_{k}$ and $x_{q(l)-k}^{l}-p^{l} \rightarrow x_{-k}$ for some configuration $\left\{x_{k}\right\}_{k \in \mathbb{Z}}$. By definition of the Aubry set, each $\Pi_{\tau}\left(x_{k}, x_{k+1}\right)$ belongs to $\mathcal{A}_{\tau}(L)$. A special care should be given in the previous argument when the length $l$ remains bounded. 


\section{Mañé potential and Peierls barrier}

We introduce in this section two new definitions: the Mañé potential and the Peierls barrier. We prove that these notions give an equivalent characterization of the Aubry set and that they give a different way to construct calibrated sub-actions. They will play a fundamental role in the next section to classify all calibrated sub-actions.

Definition 8.1. Let $L(x, v): \mathbb{T}^{d} \times \mathbb{R}^{d} \rightarrow \mathbb{R}$ be a $C^{0}$ coercive Lagrangian. We call Mañé potential the function $S_{\tau}: \mathbb{R}^{d} \times \mathbb{R}^{d} \rightarrow \mathbb{R}$ defined by

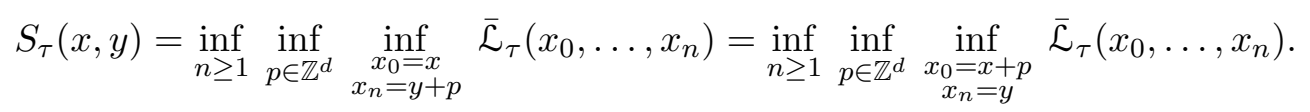

Notice that $S_{\tau}(x, y)$ is periodic in both variables $x$ and $y$.

We first give obvious properties of the Mañé potential.

Remark 8.2. For any $x, y, z$ in $\mathbb{R}^{d}$, we have

i. $S_{\tau}(x, y) \leq \inf _{p \in \mathbb{Z}^{d}}\left[\mathcal{L}_{\tau}(x, y+p)-\tau \bar{L}(\tau)\right] \leq \mathcal{L}_{\tau}(x, y)-\tau \bar{L}(\tau)=\overline{\mathcal{L}}_{\tau}(x, y)$,

ii. $u(y)-u(x) \leq S_{\tau}(x, y)$, for any sub-action $u \in C^{0}\left(\mathbb{T}^{d}\right)$,

iii. $S_{\tau}(x, y) \leq S_{\tau}(x, z)+S_{\tau}(z, y)$,

iv. $S_{\tau}(x, x) \geq 0$.

We just have seen that coerciveness implies the Mañé potential is a finite function. We show in the next propostion that $S_{\tau}(x, y)$ is continuous with respect to both $x$ and $y$.

Proposition 8.3. Let $L(x, v)$ be a $C^{0}$ coercive Lagrangian. Then

i. $S_{\tau}(x, y): \mathbb{R}^{d} \times \mathbb{R}^{d} \rightarrow \mathbb{R}$ is continuous and periodic in $x$ and $y$,

ii. For every $x, y \in \mathbb{R}^{d}, S_{\tau}(x, \cdot)$ and $-S_{\tau}(\cdot, y)$ are $C^{0}\left(\mathbb{T}^{d}\right)$ sub-actions.

Proof. We prove the first assertion. Fix $\epsilon>0$. Take arbitrary points $(x, y),\left(x^{\prime}, y^{\prime}\right)$ in $\mathbb{R}^{d} \times \mathbb{R}^{d}$. Then there exist two configurations $\left(x_{0}, \ldots, x_{m}\right),\left(y_{0}, \ldots, y_{n}\right)$ and two vectors with integer coordinates $r, s \in \mathbb{Z}^{d}$ such that

$$
\begin{aligned}
\overline{\mathcal{L}}_{\tau}\left(x_{0}, \ldots, x_{m-1}, x_{m}+r\right) & \leq S_{\tau}\left(x, y^{\prime}\right)+\epsilon / 2, \quad x_{0}=x, \quad x_{m}=y^{\prime}, \\
\overline{\mathcal{L}}_{\tau}\left(y_{0}+s, y_{1}, \ldots, y_{n}\right) & \leq S_{\tau}(x, y)+\epsilon / 2, \quad y_{0}=x, \quad y_{n}=y .
\end{aligned}
$$

Then $S_{\tau}\left(x^{\prime}, y^{\prime}\right)-S_{\tau}(x, y)=S_{\tau}\left(x^{\prime}, y^{\prime}\right)-S_{\tau}\left(x, y^{\prime}\right)+S_{\tau}\left(x, y^{\prime}\right)-S_{\tau}(x, y)$ can be bounded from above using the estimates

$$
\begin{aligned}
S_{\tau}\left(x^{\prime}, y^{\prime}\right) & -S_{\tau}\left(x, y^{\prime}\right) \leq \\
& \leq \overline{\mathcal{L}}_{\tau}\left(x^{\prime}, x_{1}, \ldots, x_{m-1}, y^{\prime}+r\right)-\overline{\mathcal{L}}_{\tau}\left(x, x_{1}, \ldots, x_{m-1}, y^{\prime}+r\right)+\epsilon / 2 \\
& \leq \overline{\mathcal{L}}_{\tau}\left(x^{\prime}, x_{1}\right)-\overline{\mathcal{L}}_{\tau}\left(x, x_{1}\right)+\epsilon / 2 \\
S_{\tau}\left(x, y^{\prime}\right) & -S_{\tau}(x, y) \leq \\
& \leq \overline{\mathcal{L}}_{\tau}\left(x+s, y_{1}, \ldots, y_{n-1}, y^{\prime}\right)-\overline{\mathcal{L}}_{\tau}\left(x+s, y_{1}, \ldots, y_{n-1}, y\right)+\epsilon / 2 \\
& \leq \overline{\mathcal{L}}_{\tau}\left(y_{n-1}, y^{\prime}\right)-\overline{\mathcal{L}}_{\tau}\left(y_{n-1}, y\right)+\epsilon / 2 .
\end{aligned}
$$


Since $S_{\tau}(x, y)$ is uniformly bounded from above by periodicity and item $i i$ of remark 8.2, lemma 7.5 guarantees that the points $x_{1}$ and $y_{n-1}$ which depend on $\epsilon$ and $x, y, x^{\prime}$ and $y^{\prime}$ are uniformly bounded. So the estimation above shows $S_{\tau}$ is a continuous map.

The second assertion is an immediate corollary of item iii of remark 8.2

$$
S_{\tau}(x, z)-S_{\tau}(x, y) \leq S_{\tau}(y, z) \leq \mathcal{L}_{\tau}(y, z)-\tau \bar{L}, \quad \forall y, z \in \mathbb{R}^{d},
$$

or in terms of the Lagranigan $L$

$$
S_{\tau}(x, y+\tau v)-S_{\tau}(x, y) \leq \tau L(x, v)-\tau \bar{L}, \quad \forall(y, v) \in \mathbb{T}^{d} \times \mathbb{R}^{d} .
$$

We just have proved that $S_{\tau}(x, \cdot)$ is a sub-action. Similarly $-S_{\tau}(\cdot, y)$ is a subaction.

For a $C^{0}$ coercive Lagrangian, we clearly deduce $S_{\tau}(x, x) \geq 0$ from item ii of remark 8.2. We show in the following proposition that $S_{\tau}(x, x)=0$ characterizes the Aubry set.

Proposition 8.4. Suppose $L(x, v)$ is a $C^{0}$ coercive Lagrangian. Then $S_{\tau}(x, x)=0$ if, and only if, $x\left(\bmod \mathbb{Z}^{d}\right) \in \operatorname{pr}^{1}\left(\mathcal{A}_{\tau}(L)\right)$.

Proof. Let us first show that $(x, v) \in \mathcal{A}_{\tau}(L)$ implies $S_{\tau}(x, x)=0$. One can find a sequence of periodic configurations $\left(x_{0}^{l}, x_{1}^{l}, \ldots, x_{q(l)}^{l}\right), x_{q(l)}^{l}=x_{0}^{l}+p^{l}$, such that

$$
x_{0}^{l} \rightarrow x, \quad x_{1}^{l} \rightarrow x+\tau v \quad \text { and } \quad \overline{\mathcal{L}}_{\tau}\left(x_{0}^{l}, x_{1}^{l}, \ldots, x_{q(l)}^{l}\right) \rightarrow 0 .
$$

Since $S_{\tau}\left(x_{0}^{l}, x_{0}^{l}\right)=S_{\tau}\left(x_{0}^{l}, x_{q(l)}^{l}\right) \leq \overline{\mathcal{L}}_{\tau}\left(x_{0}^{l}, x_{1}^{l}, \ldots, x_{q(l)}^{l}\right)$, thanks to the continuity of $S_{\tau}$ and item $i v$ of remark 8.2, we obtain $S_{\tau}(x, x)=0$.

Conversely, assume $S_{\tau}(x, x)=0$. Then there exists a sequence of periodic configurations $\left(x_{0}^{l}, \ldots, x_{q(l)}^{l}\right)$ such that $x_{0}^{l}=x=x_{q(l)}^{l}-p^{l}$ and $\overline{\mathcal{L}}_{\tau}\left(x_{0}^{l}, x_{1}^{l}, \ldots, x_{q(l)}^{l}\right) \rightarrow 0$. Thanks to lemma $7.5, x_{1}^{l}-x_{0}^{l}$ remains uniformly bounded. So one can find a subsequence of $l$ 's such that $\left\{\left(x_{1}^{l}-x_{0}^{l}\right) / \tau\right\}_{l}$ converges to some $v \in \mathbb{R}^{d}$. By definition of the Aubry set, $(x, v) \in \mathcal{A}_{\tau}(L)$.

Mañé potential enables us to construct continuous sub-actions without using the Lax-Oleinik operator. These sub-actions may not be calibrated. We introduce in the following definition a barrier which is similar to Mañé potential, being continuous, periodic with respect to both variables and in addition defining calibrated sub-actions (see theorem 8.10).

Definition 8.5. Let $L(x, v): \mathbb{T}^{d} \times \mathbb{R}^{d} \rightarrow \mathbb{R}$ be a $C^{0}$ coercive Lagrangian. We call Peierls barrier the function $\mathbf{h}_{\tau}: \mathbb{R}^{d} \times \mathbb{R}^{d} \rightarrow \mathbb{R} \cup\{+\infty\}$ defined by

$\mathbf{h}_{\tau}(x, y)=\liminf _{n \rightarrow+\infty} \inf _{p \in \mathbb{Z}^{d}} \inf _{\substack{x_{0}=x \\ x_{n}=y+p}} \overline{\mathcal{L}}_{\tau}\left(x_{0}, \ldots, x_{n}\right)=\liminf _{n \rightarrow+\infty} \inf _{p \in \mathbb{Z}^{d}} \inf _{\substack{x_{0}=x+p \\ x_{n}=y}} \overline{\mathcal{L}}_{\tau}\left(x_{0}, \ldots, x_{n}\right)$.

Again notice that $\mathbf{h}_{\tau}(x, y)$ is periodic with respect to both variables $x$ and $y$.

We first gather simple properties of the Peierls barrier. 
Remark 8.6. For any $x, y, z$ in $\mathbb{R}^{d}$

$$
\begin{aligned}
\text { i. } S_{\tau}(x, y) & \leq \mathbf{h}_{\tau}(x, y), \\
\text { ii. } \mathbf{h}_{\tau}(x, y) & \leq S_{\tau}(x, z)+\mathbf{h}_{\tau}(z, y), \\
\text { iii. } \mathbf{h}_{\tau}(x, y) & \leq \mathbf{h}_{\tau}(x, z)+S_{\tau}(z, y) .
\end{aligned}
$$

We will prove in a moment that $\mathbf{h}_{\tau}(x, y)$ satisfies additional properties: $\mathbf{h}_{\tau}(x, y)$ takes finite values (proposition 8.7), $\mathbf{h}_{\tau}(x, \cdot)$ and $-\mathbf{h}_{\tau}(\cdot, y)$ are continuous, periodic calibrated sub-actions for all $x, y \in \mathbb{R}^{d}$ (theorem 8.10).

We first prove that $S_{\tau}$ and $\mathbf{h}_{\tau}$ coincide on the projected Aubry set.

Proposition 8.7. Let $L(x, v)$ be a $C^{0}$ coercive Lagrangian. Then for any points $x, y\left(\bmod \mathbb{Z}^{d}\right) \in \operatorname{pr}^{1}\left(\mathcal{A}_{\tau}(L)\right), S_{\tau}(x, \cdot)=\mathbf{h}_{\tau}(x, \cdot)$ and $S_{\tau}(\cdot, y)=\mathbf{h}_{\tau}(\cdot, y)$. In particular $\mathbf{h}_{\tau}(x, y)$ is finite for all $x, y \in \mathbb{R}^{d}$.

Proof. We only prove the first identity. Let $x\left(\bmod \mathbb{Z}^{d}\right) \in \operatorname{pr}^{1}\left(\mathcal{A}_{\tau}(L)\right)$ and $y \in \mathbb{R}^{d}$. For every $\epsilon>0$, there exists a configuration $\left(x, y_{1}, \ldots, y_{m-1}, y+s\right)$ in $\mathbb{R}^{d}$, with $m \geq 1$ and $s \in \mathbb{Z}^{d}$, such that

$$
\overline{\mathcal{L}}_{\tau}\left(x, y_{1}, \ldots, y_{m-1}, y+s\right)<S_{\tau}(x, y)+\epsilon / 2 .
$$

As $S_{\tau}(x, x)=0$, for every positive integer $l$, one can also find a finite configuration $\left(x, x_{1}, \ldots, x_{n-1}, x+r\right)$, with $n \geq 1$ and $r \in \mathbb{Z}^{d}$, such that

$$
\overline{\mathcal{L}}_{\tau}\left(x, x_{1}, \ldots, x_{n-1}, x+r\right)<\epsilon / 2 l .
$$

Notice that the configuration

$$
\begin{aligned}
& \left(x, x_{1}, \ldots, x_{n-1}, x+r, x_{1}+r, \ldots, x_{n-1}+r, x+2 r, x_{1}+2 r, \ldots, x_{n-1}+2 r, \ldots\right. \\
& \left.x+(l-1) r, \ldots, x_{n-1}+(l-1) r, x+r l, y_{1}+l r, \ldots, y_{m-1}+l r, y+l r+s\right)
\end{aligned}
$$

is of the form $\left(z_{0}, z_{1}, \ldots, z_{n l+m}\right)$ satisfying $z_{0}=x, z_{n l+m}=y+l r+s$ and

$$
\overline{\mathcal{L}}_{\tau}\left(z_{0}, \ldots, z_{n l+m}\right) \leq l \overline{\mathcal{L}}_{\tau}\left(x, x_{1}, \ldots, x_{n-1}, x\right)+\overline{\mathcal{L}}_{\tau}\left(y, y_{1}, \ldots, y_{m-1}, y\right) \leq S_{\tau}(x, y)+\epsilon .
$$

Since $l$ can be chosen arbitrarily large, we deduce that $\mathbf{h}_{\tau}(x, y) \leq S_{\tau}(x, y)+\epsilon$, which immediately yields $\mathbf{h}_{\tau}(x, y) \leq S_{\tau}(x, y)$.

The fact that $\mathbf{h}_{\tau}(x, y)$ is finite comes from the inequality

$$
\mathbf{h}_{\tau}(x, y) \leq S_{\tau}(x, z)+\mathbf{h}_{\tau}(z, y)=S_{\tau}(x, z)+S_{\tau}(z, y),
$$

where $z \in \operatorname{pr}^{1}\left(\mathcal{A}_{\tau}(L)\right)$ is arbitrarily chosen.

Thanks to proposition 8.3, we conclude that $\mathbf{h}_{\tau}(x, \cdot)$ and $-\mathbf{h}_{\tau}(\cdot, y)$ are continuous, $\mathbb{Z}^{d}$-periodic sub-actions with respect to $L$ as soon as $x, y \in \operatorname{pr}^{1}\left(\mathcal{A}_{\tau}(L)\right)$. As a matter of fact, they are also calibrated sub-actions on the projected Aubry set (which is a first step in the proof of theorem 8.10). 
Proposition 8.8. Let $L(x, v)$ be a $C^{0}$ coercive Lagrangian.

i. For any $x\left(\bmod \mathbb{Z}^{d}\right) \in \operatorname{pr}^{1}\left(\mathcal{A}_{\tau}(L)\right), S_{\tau}(x, \cdot)$ is backward calibrated.

ii. For any $y\left(\bmod \mathbb{Z}^{d}\right) \in \operatorname{pr}^{1}\left(\mathcal{A}_{\tau}(L)\right),-S_{\tau}(\cdot, y)$ is forward calibrated.

Proof. We need to show that, for every $y^{\prime} \in \mathbb{R}^{d}$, there exists $y \in \mathbb{R}^{d}$ satisfying

$$
\mathbf{h}_{\tau}\left(x, y^{\prime}\right)=\mathbf{h}_{\tau}(x, y)+\overline{\mathcal{L}}_{\tau}\left(y, y^{\prime}\right) .
$$

Since $\mathbf{h}_{\tau}(x, \cdot)$ is a sub-action, we already know that $\mathbf{h}_{\tau}\left(x, y^{\prime}\right) \leq \mathbf{h}_{\tau}(x, y)+\overline{\mathcal{L}}_{\tau}\left(y, y^{\prime}\right)$. Conversely, one can find a sequence of configurations in $\mathbb{R}^{d},\left(x_{0}^{k}, x_{1}^{k}, \ldots, x_{n(k)}^{k}\right)$, such that $x_{0}^{k}=x+p^{k}$ for some $p^{k} \in \mathbb{Z}^{d}, x_{n(k)}^{k}=y^{\prime}$,

$$
n(k) \rightarrow+\infty \quad \text { and } \quad \overline{\mathcal{L}}_{\tau}\left(x_{0}^{k}, x_{1}^{k}, \ldots, x_{n(k)}^{k}\right) \rightarrow \mathbf{h}_{\tau}\left(x, y^{\prime}\right) .
$$

Thanks to lemma 7.5, a subsequence of $\left\{x_{n(k)-1}^{k}\right\}_{k}$ converges to some $y \in \mathbb{R}^{d}$. Then

$$
\begin{aligned}
\mathbf{h}_{\tau}\left(x, x_{n(k)-1}^{k}\right)+\overline{\mathcal{L}}_{\tau}\left(x_{n(k)-1}^{k}, y^{\prime}\right) & \leq S_{\tau}\left(x, x_{n(k)-1}^{k}\right)+\overline{\mathcal{L}}_{\tau}\left(x_{n(k)-1}^{k}, y^{\prime}\right) \\
& \leq \overline{\mathcal{L}}_{\tau}\left(x_{0}^{k}, x_{1}^{k}, \ldots, x_{n(k)}^{k}\right) .
\end{aligned}
$$

Letting $k$ go to $+\infty$, we obtain $\mathbf{h}_{\tau}(x, y)+\overline{\mathcal{L}}_{\tau}\left(y, y^{\prime}\right) \leq \mathbf{h}_{\tau}\left(x, y^{\prime}\right)$. In an analogous way, we can prove that $-\mathbf{h}_{\tau}(\cdot, x)=-S_{\tau}(\cdot, x)$ is a forward calibrated sub-action.

We have seen that $S_{\tau}(x, \cdot)$ and $-S_{\tau}(\cdot, y)$ are continuous, periodic sub-actions for any $x, y \in \mathbb{R}^{d}$. The following proposition shows that the Peierls barrier can be defined using Mañé potential. (That fact will be used in the proof of theorem 8.10.)

Proposition 8.9. Assume $L(x, v)$ is a $C^{0}$ coercive Lagrangian. Then

$$
\mathbf{h}_{\tau}(x, y)=\min _{z \in p r^{1}\left(\mathcal{A}_{\tau}(L)\right)}\left[S_{\tau}(x, z)+S_{\tau}(z, y)\right], \quad \forall x, y \in \mathbb{T}^{d} .
$$

Proof. Proposition 8.7 tells us $\mathbf{h}_{\tau}(\cdot, y)=S_{\tau}(\cdot, y)$ and $\mathbf{h}_{\tau}(x, \cdot)=S_{\tau}(x, \cdot)$ whenever $x, y \in \operatorname{pr}^{1}\left(\mathcal{A}_{\tau}(L)\right)$. Hence, from item ii of remark 8.6, we immediately get

$$
\mathbf{h}_{\tau}(x, y) \leq \min _{z \in r^{1}\left(\mathcal{A}_{\tau}(L)\right)}\left[S_{\tau}(x, z)+S_{\tau}(z, y)\right] .
$$

So it suffices to find $z \in \operatorname{pr}^{1}\left(\mathcal{A}_{\tau}(L)\right)$ satisfying $S_{\tau}(x, z)+S_{\tau}(z, y) \leq \mathbf{h}_{\tau}(x, y)$. Let $u$ be a $C^{0}\left(\mathbb{T}^{d}\right)$ sub-action. By taking $L(x, v)-\frac{1}{\tau}[u(x+\tau v)-u(x)]-\bar{L}(\tau)$, we may assume $L \geq 0$ and $\bar{L}(\tau)=0$. Let $\overline{\mathcal{L}}_{\tau}(x, y)=\tau L\left(x, \frac{1}{\tau}(y-x)\right)$ for $x, y \in \mathbb{R}^{d}$.

By definition of $\mathbf{h}_{\tau}(x, y)$, there exists a sequence of configurations $\left(x_{0}^{k}, \ldots, x_{n(k)}^{k}\right)$ in $\mathbb{R}^{d}$ of length $n(k)$ and a sequence $p^{k} \in \mathbb{Z}^{d}$ such that $x_{0}^{k}=x, x_{n(k)}^{k}=y+p^{k}$,

$$
n(k) \rightarrow+\infty \text { and } \quad \lim _{k \rightarrow \infty} \overline{\mathcal{L}}_{\tau}\left(x_{0}^{k}, \ldots, x_{n(k)}^{k}\right)=\mathbf{h}_{\tau}(x, y) .
$$

Since $\mathbf{h}_{\tau}(x, y)<\infty$ and $\overline{\mathcal{L}}_{\tau} \geq 0$, for $k$ large enough, one can find $m(k)$ and $m^{\prime}(k)$ in $\{0, \ldots, n(k)-1\}$ such that

$$
m^{\prime}(k)-m(k)=\left\lfloor\sqrt{n_{k}}\right\rfloor \quad \text { and } \quad 0 \leq \overline{\mathcal{L}}_{\tau}\left(x_{m(k)}^{k}, \ldots, x_{m^{\prime}(k)}^{k}\right)<\frac{\mathbf{h}_{\tau}(x, y)+1}{\left\lfloor\sqrt{n_{k}}\right\rfloor-1} .
$$


Otherwise, we would reach a contradiction, because for arbitrarily large $k$

$$
\begin{aligned}
\overline{\mathcal{L}}_{\tau}\left(x_{0}^{k}, \ldots, x_{n_{k}}^{k}\right) & \geq \sum_{i=0}^{\left\lfloor\sqrt{n_{k}}\right\rfloor-2} \overline{\mathcal{L}}_{\tau}\left(x_{i\left\lfloor\sqrt{n_{k}}\right\rfloor}^{k}, \ldots, x_{(i+1)\left\lfloor\sqrt{n_{k}}\right\rfloor}^{k}\right) \\
& \geq\left(\left\lfloor\sqrt{n_{k}}\right\rfloor-1\right) \frac{\mathbf{h}_{\tau}(x, y)+1}{\left\lfloor\sqrt{n_{k}}\right\rfloor-1}=\mathbf{h}_{\tau}(x, y)+1 .
\end{aligned}
$$

Thanks to the invariance of $\mathcal{L}_{\tau}$ by the diagonal action of $\mathbb{Z}^{d}, \mathcal{L}_{\tau}(x+s, y+s)=$ $\mathcal{L}_{\tau}(x, y)$ for all $s \in \mathbb{Z}^{d}$, we may assume $x_{m(k)}^{k} \in[0,1)^{d}$. Using a diagonal procedure, lemma 7.5 allows us to find a subsequence $\left\{k_{j}\right\}$ of integers and a forward infinite configuration $\left\{z_{l}\right\}_{l \geq 0}$ of $\mathbb{R}^{d}$ such that

$$
\lim _{j \rightarrow \infty} x_{m\left(k_{j}\right)}^{k_{j}}=z_{0} \in[0,1)^{d} \quad \text { and } \quad \lim _{j \rightarrow \infty} x_{m\left(k_{j}\right)+l}^{k_{j}}=z_{l} \in \mathbb{R}^{d}, \quad \forall l \geq 1 .
$$

From the construction of the sequence $\left\{m_{k}\right\}$, it follows that $\mathcal{L}_{\tau}\left(z_{l}, z_{l+1}\right)=0$ for any nonnegative integer $l$, which clearly yields $S_{\tau}\left(z_{l}, z_{l+1}\right)=0$. From item iii of remark 8.2, we get $S_{\tau}\left(z_{l}, z_{l^{\prime}}\right)=0$ whenever $l^{\prime}>l \geq 0$. Therefore, if $z_{\infty} \in \mathbb{T}^{d}$ is an arbitrary accumulation point of $\left\{z_{l}\left(\bmod \mathbb{Z}^{d}\right)\right\}_{l \geq 0}$, then $S_{\tau}\left(z_{\infty}, z_{\infty}\right)=0$ or $z_{\infty} \in \operatorname{pr}^{1}\left(\mathcal{A}_{\tau}(L)\right)$. Observe that, for any $l \geq 0$,

$$
\begin{aligned}
\overline{\mathcal{L}}_{\tau}\left(x_{0}^{k_{j}}, \ldots, x_{n\left(k_{j}\right)}^{k_{j}}\right) & =\overline{\mathcal{L}}_{\tau}\left(x_{0}^{k_{j}}, \ldots, x_{m\left(k_{j}\right)+l}^{k_{j}}\right)+\overline{\mathcal{L}}_{\tau}\left(x_{m\left(k_{j}\right)+l}^{k_{j}}, \ldots, x_{n\left(k_{j}\right)}^{k_{j}}\right) \\
& \geq S_{\tau}\left(x, x_{m\left(k_{j}\right)+l}^{k_{j}}\right)+S_{\tau}\left(x_{m\left(k_{j}\right)+l}^{k_{j}}, y\right) .
\end{aligned}
$$

Passing to the limit when $j \rightarrow \infty$, we obtain $\mathbf{h}_{\tau}(x, y) \geq S_{\tau}\left(x, z_{l}\right)+S_{\tau}\left(z_{l}, y\right)$. Taking then a suitable subsequence of $\left\{z_{l}\right\}$, we get $\mathbf{h}_{\tau}(x, y) \geq S_{\tau}\left(x, z_{\infty}\right)+S_{\tau}\left(z_{\infty}, y\right)$.

Theorem 8.10. Let $L(x, v)$ be a $C^{0}$ coercive Lagrangian. Then the Peierls barrier $\mathbf{h}_{\tau}: \mathbb{R}^{d} \times \mathbb{R}^{d} \rightarrow \mathbb{R}$ is continuous, $\mathbb{Z}^{d} \times \mathbb{Z}^{d}$ periodic. Moreover, $\mathbf{h}_{\tau}(x, \cdot): \mathbb{R}^{d} \rightarrow \mathbb{R}$ is a forward calibrated sub-action and $-\mathbf{h}_{\tau}(\cdot, y): \mathbb{R}^{d} \rightarrow \mathbb{R}$ is a backward calibrated sub-action for any $x, y \in \mathbb{R}^{d}$.

Proof. Consider arbitrary points $(x, y),\left(x^{\prime}, y^{\prime}\right) \in \mathbb{T}^{d} \times \mathbb{T}^{d}$. Thanks to proposition 8.9, there exists $z_{x, y} \in p r^{1}\left(\mathcal{A}_{\tau}(L)\right)$ satisfying $\mathbf{h}_{\tau}(x, y)=S_{\tau}\left(x, z_{x, y}\right)+S_{\tau}\left(z_{x, y}, y\right)$. Then

$$
\mathbf{h}_{\tau}\left(x^{\prime}, y^{\prime}\right)-\mathbf{h}_{\tau}(x, y) \leq\left[S_{\tau}\left(x^{\prime}, z_{x, y}\right)-S_{\tau}\left(x, z_{x, y}\right)\right]+\left[S_{\tau}\left(z_{x, y}, y^{\prime}\right)-S_{\tau}\left(z_{x, y}, y\right)\right] .
$$

Since $S_{\tau}$ is uniformly continuous on $\mathbb{T}^{d} \times \mathbb{T}^{d}$, the estimation above assures that $\mathbf{h}_{\tau}$ is a continuous map.

We already know that $\mathbf{h}_{\tau}(x, \cdot)$ and $-\mathbf{h}_{\tau}(\cdot, x)$ are $\mathbb{T}^{d}$ periodic and continuous. Take $\left(y, y^{\prime}\right) \in \mathbb{R}^{d} \times \mathbb{R}^{d}$. Thanks to proposition 8.9, there exists $z \in \operatorname{pr}^{1}\left(\mathcal{A}_{\tau}(L)\right)$ such that $\mathbf{h}_{\tau}(x, y)=S_{\tau}(x, z)+S_{\tau}(z, y)$. Then, using the fact that $S_{\tau}(z, \cdot)$ is a sub-action

$$
\mathbf{h}_{\tau}\left(x, y^{\prime}\right)-\mathbf{h}_{\tau}(x, y) \leq S_{\tau}\left(z, y^{\prime}\right)-S_{\tau}(z, y) \leq \overline{\mathcal{L}}\left(y, y^{\prime}\right) .
$$

We have proved that $\mathbf{h}_{\tau}(x, \cdot)$ is a sub-action. Since $S_{\tau}(z, \cdot)$ is also backward calibrated, one can find $y^{\prime \prime} \in \mathbb{R}^{d}$ such that $S_{\tau}(z, y)=S_{\tau}\left(z, y^{\prime \prime}\right)+\overline{\mathcal{L}}\left(y^{\prime \prime}, y\right)$. Then

$$
\mathbf{h}_{\tau}(x, y)=S_{\tau}(x, z)+S_{\tau}\left(z, y^{\prime \prime}\right)+\overline{\mathcal{L}}\left(y^{\prime \prime}, y\right) \geq \mathbf{h}_{\tau}\left(x, y^{\prime \prime}\right)+\overline{\mathcal{L}}\left(y^{\prime \prime}, y\right) .
$$

We have proved that $\mathbf{h}_{\tau}(x, \cdot)$ is calibrated. Analogously, one can show that $-\mathbf{h}_{\tau}(\cdot, y)$ is a calibrated sub-action too. 


\section{Representation formulas for calibrated sub-actions}

In the continuous-time Aubry-Mather theory, Contreras has characterized in [16] the set of weak KAM solutions of the Hamilton-Jacobi equation in terms of their values at each static class. We remark that weak KAM solutions are similar to calibrated sub-actions and the set of static classes is similar to the projected Aubry set. We show in the following theorem how a calibrated sub-action is completely known when it is known on the Aubry set. In the context of ergodic optimization for symbolic dynamics, a similar result has been proved in [31].

Theorem 9.1. Let $L(x, v)$ be a $C^{0}$ coercive Lagrangian. If $u_{+} \in C^{0}\left(\mathbb{T}^{d}\right)$ is a forward calibrated sub-action or $u_{-} \in C^{0}\left(\mathbb{T}^{d}\right)$ is a backward calibrated sub-action, then, for every $x, y \in \mathbb{R}^{d}$,

$$
\begin{aligned}
& u_{+}(x)=\max _{y \in p r^{1}\left(\mathcal{A}_{\tau}(L)\right)}\left[u_{+}(y)-S_{\tau}(x, y)\right]=\max _{y \in p r^{1}\left(\mathcal{A}_{\tau}(L)\right)}\left[u_{+}(y)-\mathbf{h}_{\tau}(x, y)\right], \\
& u_{-}(y)=\min _{x \in p r^{1}\left(\mathcal{A}_{\tau}(L)\right)}\left[u_{-}(x)+S_{\tau}(x, y)\right]=\min _{x \in p r^{1}\left(\mathcal{A}_{\tau}(L)\right)}\left[u_{-}(x)+\mathbf{h}_{\tau}(x, y)\right] .
\end{aligned}
$$

Proof. Thanks to proposition 8.7, we just need to prove the two first equalities. From item $i i$ of remark 8.2 , we verify without difficulty that

$$
u_{+}(x) \geq \max _{y \in p r^{1}\left(\mathcal{A}_{\tau}(L)\right)}\left[u_{+}(y)-S_{\tau}(x, y)\right] .
$$

As $u_{+}$is a forward calibrated sub-action, one can find a forward configuration $\left\{x_{k}\right\}_{k \geq 0}$ of $\mathbb{R}^{d}$ such that $x_{0}=x$ and $u_{+}\left(x_{k}\right)=u_{+}\left(x_{k+1}\right)-\overline{\mathcal{L}}_{\tau}\left(x_{k}, x_{k+1}\right)$ for every $k \geq$ 0 . From $u_{+}\left(x_{l}\right)-u_{+}\left(x_{k}\right) \leq S_{\tau}\left(x_{k}, x_{l}\right) \leq \overline{\mathcal{L}}_{\tau}\left(x_{k}, \ldots, x_{l}\right)=u_{+}\left(x_{l}\right)-u_{+}\left(x_{k}\right)$ whenever $l>k \geq 0$, we conclude that $S_{\tau}\left(x_{k}, x_{l}\right)=u_{+}\left(x_{l}\right)-u_{+}\left(x_{k}\right)$. Therefore, if $y \in \mathbb{T}^{d}$ is an arbitrary accumulation point of $\left\{x_{k}\left(\bmod \mathbb{Z}^{d}\right)\right\}$, it follows that $S_{\tau}(y, y)=0$, namely, $y \in \operatorname{pr}^{1}\left(\mathcal{A}_{\tau}(L)\right)$. Furthermore, by taking a suitable subsequence, $u_{+}(x)=$ $u_{+}\left(x_{k}\right)-S_{\tau}\left(x, x_{k}\right)$ tends to $u_{+}(x)=u_{+}(y)-S_{\tau}(x, y)$.

Analogously, one can demonstrate the existence of a point $x \in \operatorname{pr}^{1}\left(\mathcal{A}_{\tau}(L)\right)$ achieving $u_{-}(y)=u_{-}(x)+S_{\tau}(x, y)$.

Corollary 9.2. Suppose $u, u^{\prime} \in C^{0}\left(\mathbb{T}^{d}\right)$ are both either forward or backward calibrated sub-actions with respect to a $C^{0}$ coercive Lagrangian $L(x, v)$.

$$
\begin{aligned}
& \text { i. If }\left.u\right|_{p r^{1}\left(\mathcal{A}_{\tau}(L)\right)} \leq\left. u^{\prime}\right|_{p r^{1}\left(\mathcal{A}_{\tau}(L)\right)} \text {, then } u \leq u^{\prime} \text { everywhere on } \mathbb{R}^{d} . \\
& \text { ii. If }\left.u\right|_{p r^{1}\left(\mathcal{A}_{\tau}(L)\right)}=\left.u^{\prime}\right|_{p r^{1}\left(\mathcal{A}_{\tau}(L)\right)} \text {, then } u=u^{\prime} \text { everywhere on } \mathbb{R}^{d} \text {. }
\end{aligned}
$$

Theorem 9.1 admits a reciprocal.

Theorem 9.3. Let $L(x, v)$ be a $C^{0}$ coercive Lagrangian and $\psi$ be any function defined on the projected Aubry set $\operatorname{pr}^{1}\left(\mathcal{A}_{\tau}(L)\right)$.

$i$. If $\psi$ is bounded above, the following $u$ is a continuous forward calibrated subaction

$$
u(x):=\sup _{y \in p r^{1}\left(\mathcal{A}_{\tau}(L)\right)}\left[\psi(y)-S_{\tau}(x, y)\right]=\sup _{y \in p r^{1}\left(\mathcal{A}_{\tau}(L)\right)}\left[\psi(y)-\mathbf{h}_{\tau}(x, y)\right] .
$$


ii. If $\psi$ is bounded below, the following $u$ is a continuous backward calibrated sub-action

$$
u(y):=\inf _{x \in p r^{1}\left(\mathcal{A}_{\tau}(L)\right)}\left[\psi(x)+S_{\tau}(x, y)\right]=\inf _{x \in p r^{1}\left(\mathcal{A}_{\tau}(L)\right)}\left[\psi(x)+\mathbf{h}_{\tau}(x, y)\right] .
$$

iii. If $\psi(y)-\psi(x) \leq S_{\tau}(x, y)$ for all $x, y \in p r^{1}\left(\mathcal{A}_{\tau}(L)\right)$, then $\left.u\right|_{p r^{1}\left(\mathcal{A}_{\tau}\right)}=\psi$.

Proof. In any case, $u: \mathbb{R}^{d} \rightarrow \mathbb{R}$ is clearly a well defined periodic function. Since both constructions are similar, we will discuss just the second one. So let us show that $u \in C^{0}\left(\mathbb{T}^{d}\right)$. Fix $\epsilon>0$ and consider $y, y^{\prime} \in \mathbb{R}^{d}$. Take $x\left(\bmod \mathbb{Z}^{d}\right) \in p r^{1}\left(\mathcal{A}_{\tau}(L)\right)$ such that $\psi(x)+S_{\tau}(x, y)<u(y)+\epsilon$. Thus $u\left(y^{\prime}\right)-u(y) \leq S_{\tau}\left(x, y^{\prime}\right)-S_{\tau}(x, y)+\epsilon$. Since $S_{\tau}$ is uniformly continuous on $\mathbb{R}^{d} \times \mathbb{R}^{d}$ and $\epsilon>0$ is arbitrary, it is easy to deduce that

$$
\left|u\left(y^{\prime}\right)-u(y)\right| \leq \max _{x \in \operatorname{pr}^{1}\left(\mathcal{A}_{\tau}(L)\right)}\left|S_{\tau}\left(x, y^{\prime}\right)-S_{\tau}(x, y)\right|,
$$

which guarantees the continuity of $u$.

We now show that $u$ is backward calibrated. Given $y \in \mathbb{R}^{d}$ and $\epsilon>0$, choose $x \in \operatorname{pr}^{1}\left(\mathcal{A}_{\tau}(L)\right)$ satisfying $\psi(x)+S_{\tau}(x, y)<u(y)+\epsilon$. Thanks to proposition 8.3, $S_{\tau}(x, \cdot)$ is a sub-action and

$$
u(y+\tau w)-u(y)-\epsilon<S_{\tau}(x, y+\tau w)-S_{\tau}(x, y) \leq \tau L(y, w)-\tau \bar{L}(\tau), \quad \forall w \in \mathbb{R}^{d} .
$$

Letting $\epsilon$ go to 0 , we obtain that $u$ is a sub-action. To prove that $u$ is a calibrated sub-action, we use the fact that the sub-actions $\left\{S_{\tau}(x, \cdot)\right\}_{x \in p r^{1}\left(\mathcal{A}_{\tau}(L)\right)}$ are calibrated (see proposition 8.8). Let $y \in \mathbb{R}^{d}$. It suffices to show there exists $v \in \mathbb{R}^{d}$ such that $u(y) \geq u(y-\tau v)+\tau L(y-\tau v, v)-\tau \bar{L}(\tau)$. By definiton of $u(y)$, there exists a sequence of points $x_{k} \in \operatorname{pr}^{1}\left(\mathcal{A}_{\tau}(L)\right)$ such that $\psi\left(x_{k}\right)+S_{\tau}\left(x_{k}, y\right)<u(y)+\frac{1}{k}$. Moreover, there exists a sequence of $v_{k} \in \mathbb{R}^{d}$ such that

$$
S_{\tau}\left(x_{k}, y\right)=S_{\tau}\left(x_{k}, y-\tau v_{k}\right)+\tau L\left(y-\tau v_{k}, v_{k}\right)-\tau \bar{L}(\tau) .
$$

Remember we can assume $\left\|v_{k}\right\|_{\infty} \leq R_{\tau}$ (see lemma 5.5) for some constant $R_{\tau}>\frac{1}{\tau}$ (see definition 3.8). Let $v \in \mathbb{R}^{d}$ be an accumulation point of the sequence $\left\{v_{k}\right\}_{k \geq 0}$. Since $u\left(y-\tau v_{k}\right) \leq \psi\left(x_{k}\right)+S_{\tau}\left(x_{k}, y-\tau v_{k}\right)$, we obtain

$$
u\left(y-\tau v_{k}\right)+\tau L\left(y-\tau v_{k}, v_{k}\right)-\tau \bar{L}(\tau)<u(y)+\frac{1}{k} .
$$

Taking a suitable subsequence, we get $u(y-\tau v)+\tau L(y-\tau v, v)-\tau \bar{L}(\tau) \leq u(y)$.

Suppose $\psi(y)-\psi(x) \leq S_{\tau}(x, y)$ for all $x, y \in p r^{1}\left(\mathcal{A}_{\tau}(L)\right)$ and $u$ is defined as in item ii. Let $y\left(\bmod \mathbb{Z}^{d}\right) \in \operatorname{pr}^{1}\left(\mathcal{A}_{\tau}(L)\right)$. On the one hand, $u(y) \leq \psi(y)$ by taking $x=y$ in the definition of $u$ and noticing that $S_{\tau}(y, y)=0$. On the other hand, for any $x \in \operatorname{pr}^{1}\left(\mathcal{A}_{\tau}(L)\right), S_{\tau}(x, y)+\psi(x) \geq \psi(y)$ by hypothesis on $\psi$. By taking the infimum on $x$ we obtain $u(y) \geq \psi(y)$. We have proved that $\left.u\right|_{p r^{1}\left(\mathcal{A}_{\tau}(L)\right)}=\psi$.

Thanks to item ii of remark 8.2, an immediate but important consequence of theorem 9.3 is the fact that the restriction of any sub-action to the projected Aubry set behaves as a forward or backward calibrated sub-action. 
Corollary 9.4. Let $u \in C^{0}\left(\mathbb{T}^{d}\right)$ be an arbitrary sub-action for a $C^{0}$ coercive Lagrangian $L(x, v)$. Then, for every point $x \in \operatorname{pr}^{1}\left(\mathcal{A}_{\tau}(L)\right)$, we have

$u(x)=\max _{v \in \mathbb{R}^{d}}[u(x+\tau v)-\tau L(x, v)+\tau \bar{L}(\tau)]=\min _{v \in \mathbb{R}^{d}}[u(x-\tau v)+\tau L(x-\tau v, v)-\tau \bar{L}(\tau)]$.

Theorem 9.3 motivates the introduction of the following notion.

Definition 9.5. Let $L(x, v)$ be a $C^{0}$ coercive Lagrangian. Suppose that $u_{+}$is a $C^{0}\left(\mathbb{T}^{d}\right)$ forward calibrated sub-action and that $u_{-}$is a $C^{0}\left(\mathbb{T}^{d}\right)$ backward calibrated sub-action. We say that $u_{+}$and $u_{-}$are conjugated sub-actions, and we use the notation $u_{+} \sim u_{-}$, if $\left.u_{+}\right|_{p r^{1}\left(\mathcal{A}_{\tau}(L)\right)}=\left.u_{-}\right|_{p r^{1}\left(\mathcal{A}_{\tau}(L)\right)}$.

Notice that coerciveness is a sufficient condition for the existence calibrated sub-actions. Moreover, corollary 9.2 implies that, given a forward calibrated subaction $u_{+}$, there exists at most one backward calibrated $u_{-}$conjugated to $u_{+}$and vice versa. Finally, theorem 9.3 shows that such a backward calibrated sub-action does exist. More precisely, if $u_{-}$is given, the conjugated $u_{+}$takes necessarily the form

$$
u_{+}(x):=\max _{y \in p r^{1}\left(\mathcal{A}_{\tau}(L)\right)}\left[u_{-}(y)-S_{\tau}(x, y)\right]
$$

and conversely if $u_{+}$is given, the conjugated $u_{-}$has the form

$$
u_{-}(x):=\min _{y \in p r^{1}\left(\mathcal{A}_{\tau}(L)\right)}\left[u_{+}(y)+S_{\tau}(y, x)\right] .
$$

Fathi points out in [22] that, for a continuous-time, autonomous, strictly convex, superlinear $C^{3}$-Lagrangian on a compact $C^{\infty}$ manifold without boundary, the Peierls barrier admits a characterization in terms of conjugated sub-actions. We obtain in the following theorem a similar result in the discrete Aubry-Mather theory.

Proposition 9.6. Let $L(x, v)$ be a $C^{0}$ coercive Lagrangian. Then

$$
\mathbf{h}_{\tau}(x, y)=\max _{u_{+} \sim u_{-}}\left[u_{-}(y)-u_{+}(x)\right], \quad \forall x, y \in \mathbb{R}^{d} .
$$

Proof. For any $z \in \operatorname{pr}^{1}\left(\mathcal{A}_{\tau}(L)\right)$ and a pair of sub-actions $u_{+}$and $u_{-}$, we have

$$
u_{+}(z)-u_{+}(x) \leq S_{\tau}(x, z) \quad \text { and } \quad u_{-}(y)-u_{-}(z) \leq S_{\tau}(z, y), \quad \forall x, y \in \mathbb{R}^{d} .
$$

If $u_{+} \sim u_{-}$are conjugated then $u_{+}(z)=u_{-}(z)$ and we obtain

$$
u_{-}(y)-u_{+}(x) \leq S_{\tau}(x, z)+S_{\tau}(z, y), \quad \forall z \in p r^{1}\left(\mathcal{A}_{\tau}(L)\right) .
$$

Thanks to proposition 8.9 , we get $u_{-}(y)-u_{+}(x) \leq \mathbf{h}_{\tau}(x, y)$, which obviously yields $\sup _{u_{+} \sim u_{-}}\left[u_{-}(y)-u_{+}(x)\right] \leq \mathbf{h}_{\tau}(x, y)$.

Fix $x, y \in \mathbb{R}^{d}$. Consider then the forward calibrated sub-action $u_{+}=-\mathbf{h}_{\tau}(\cdot, y)$ and define a backward calibrated sub-action $u_{-} \in C^{0}\left(\mathbb{T}^{d}\right)$ by

$$
u_{-}\left(x^{\prime}\right):=\min _{z \in p r^{1}\left(\mathcal{A}_{\tau}(L)\right)}\left[u_{+}(z)+S_{\tau}\left(z, x^{\prime}\right)\right]=\min _{z \in p r^{1}\left(\mathcal{A}_{\tau}(L)\right)}\left[-S_{\tau}(z, y)+S_{\tau}\left(z, x^{\prime}\right)\right] .
$$

By construction, $u_{+}$and $u_{-}$are conjugated sub-actions. Furthermore, $u_{-}(y)=0$. Thus $u_{-}(y)-u_{+}(x)=\mathbf{h}_{\tau}(x, y)$. 
Theorems 9.1 and 9.3 together provide an interesting description of the calibrated sub-actions. In order to present it, we decided to adopt a slightly different point of view.

Definition 9.7. Let $L(x, v)$ be a $C^{0}$ coercive Lagrangian. We call positive-time Mañé-Peierls transform the application $\mathcal{F}_{+}$defined on $C^{0}\left(\operatorname{pr}^{1}\left(\mathcal{A}_{\tau}(L)\right)\right)$ by

$\mathcal{F}_{+}(\psi)(x)=\max _{y \in p r^{1}\left(\mathcal{A}_{\tau}(L)\right)}\left[\psi(y)-S_{\tau}(x, y)\right]=\max _{y \in p r^{1}\left(\mathcal{A}_{\tau}(L)\right)}\left[\psi(y)-\mathbf{h}_{\tau}(x, y)\right], \quad \forall x \in \mathbb{R}^{d}$.

In the same way, we call negative-time Mañé-Peierls transform the application $\mathcal{F}_{-}$ defined on $C^{0}\left(p r^{1}\left(\mathcal{A}_{\tau}(L)\right)\right)$ by

$\mathcal{F}_{-}(\psi)(y)=\min _{x \in p r^{1}\left(\mathcal{A}_{\tau}(L)\right)}\left[\psi(x)+S_{\tau}(x, y)\right]=\min _{x \in p r^{1}\left(\mathcal{A}_{\tau}(L)\right)}\left[\psi(x)+\mathbf{h}_{\tau}(x, y)\right], \quad \forall y \in \mathbb{R}^{d}$.

We summarize then all the main properties of the Mañé-Peierls transforms.

Theorem 9.8. Let $L(x, v)$ be a $C^{0}$ coercive Lagrangian. Consider arbitrary functions $\psi, \psi^{\prime} \in C^{0}\left(\operatorname{pr}^{1}\left(\mathcal{A}_{\tau}(L)\right)\right)$. Then

i. $\mathcal{F}_{-}(\psi) \leq \psi \leq \mathcal{F}_{+}(\psi)$ everywhere on $\operatorname{pr}^{1}\left(\mathcal{A}_{\tau}(L)\right)$;

ii. $\psi \leq \psi^{\prime}$ implies $\mathcal{F}_{+}(\psi) \leq \mathcal{F}_{+}\left(\psi^{\prime}\right)$ and $\mathcal{F}_{-}(\psi) \leq \mathcal{F}_{-}\left(\psi^{\prime}\right)$;

iii. $\mathcal{F}_{+}(\psi)$ is a continuous forward calibrated sub-action;

$i v . \mathcal{F}_{-}(\psi)$ is a continuous backward calibrated sub-action;

v. if $\psi(y)-\psi(x) \leq S_{\tau}(x, y)$ for all $x, y \in p r^{1}\left(\mathcal{A}_{\tau}(L)\right)$, then $\mathcal{F}_{+}$and $\mathcal{F}_{-}$act as extension operators: $\left.\mathcal{F}_{+}(\psi)\right|_{p r^{1}\left(\mathcal{A}_{\tau}(L)\right)}=\psi=\left.\mathcal{F}_{-}(\psi)\right|_{p r^{1}\left(\mathcal{A}_{\tau}(L)\right)}$;

vi. if $u \in C^{0}\left(\mathbb{T}^{d}\right)$ is a forward calibrated sub-action, then

$$
\begin{aligned}
& \mathcal{F}_{+}\left(\left.u\right|_{p r^{1}\left(\mathcal{A}_{\tau}(L)\right)}\right)=u=\mathcal{F}_{+}\left(\left.\mathcal{F}_{-}\left(\left.u\right|_{p r^{1}\left(\mathcal{A}_{\tau}(L)\right)}\right)\right|_{p r^{1}\left(\mathcal{A}_{\tau}(L)\right)}\right) \text { everywhere on } \mathbb{R}^{d} \\
& \text { and } \mathcal{F}_{-}\left(\left.u\right|_{\pi\left(\mathcal{A}_{\tau}(L)\right)}\right) \text { is the unique sub-action conjugated to } u ;
\end{aligned}
$$

vii. if $u \in C^{0}\left(\mathbb{T}^{d}\right)$ is a backward calibrated sub-action, then

$$
\begin{aligned}
& \mathcal{F}_{-}\left(\left.u\right|_{p r^{1}\left(\mathcal{A}_{\tau}(L)\right)}\right)=u=\mathcal{F}_{-}\left(\left.\mathcal{F}_{+}\left(\left.u\right|_{p r^{1}\left(\mathcal{A}_{\tau}(L)\right)}\right)\right|_{p r^{1}\left(\mathcal{A}_{\tau}(L)\right)}\right) \text { everywhere on } \mathbb{R}^{d} \\
& \text { and } \mathcal{F}_{+}\left(\left.u\right|_{p r^{1}\left(\mathcal{A}_{\tau}(L)\right)}\right) \text { is the unique sub-action conjugated to } u ;
\end{aligned}
$$

viii. $\mathcal{F}_{+}$is a bijective and isometric correspondence between the set of the functions $\psi \in C^{0}\left(p r^{1}\left(\mathcal{A}_{\tau}(L)\right)\right)$ satisfying, for $x, y \in p r^{1}\left(\mathcal{A}_{\tau}(L)\right), \psi(y)-\psi(x) \leq S_{\tau}(x, y)$ and the set of continuous forward calibrated sub-actions;

ix. $\mathcal{F}_{-}$is a bijective and isometric correspondence between the set of the functions $\psi \in C^{0}\left(\pi\left(\mathcal{A}_{\tau}(L)\right)\right)$ satisfying, for $x, y \in p r^{1}\left(\mathcal{A}_{\tau}(L)\right), \psi(y)-\psi(x) \leq S_{\tau}(x, y)$ and the set of continuous backward calibrated sub-actions. 
Proof. Items $i$ and $i i$ follow immediately from the respective definitions of the Mañé-Peierls transforms. In truth, items $i i i, i v$ and $v$ can be seen as theorem 9.3 rewritten. Besides, items vi and vii result from theorems 9.1 and 9.3 without difficulty.

Since items viii and $i x$ are very similar, we will discuss just the first one. As $\mathcal{F}_{+}(\psi)=\psi$ everywhere on $\operatorname{pr}^{1}\left(\mathcal{A}_{\tau}(L)\right), \mathcal{F}_{+}$is injective. Moreover, when $u \in C^{0}\left(\mathbb{T}^{d}\right)$ is a forward calibrated sub-action, the identity $\mathcal{F}_{+}\left(\left.u\right|_{\pi\left(\mathcal{A}_{\tau}(L)\right)}\right)=u$ guarantees that $\mathcal{F}_{+}$is surjective. In fact, this correspondence is an isometry. Indeed, fixing $x \in \mathbb{R}^{d}$, there exists a point $y \in \operatorname{pr}^{1}\left(\mathcal{A}_{\tau}(L)\right)$ such that $\mathcal{F}_{+}(\psi)(x)=\psi(y)-S_{\tau}(x, y)$. Hence, one has

$$
\mathcal{F}_{+}(\psi)(x)-\mathcal{F}_{+}\left(\psi^{\prime}\right)(x) \leq \psi(y)-\psi^{\prime}(y) \leq\left\|\psi-\psi^{\prime}\right\|_{0} .
$$

Since $x \in \mathbb{R}^{d}$ is arbitrary and since we can interchange the roles of $\psi$ and $\psi^{\prime}$, we get $\left\|\mathcal{F}_{+}(\psi)-\mathcal{F}_{+}\left(\psi^{\prime}\right)\right\|_{0} \leq\left\|\psi-\psi^{\prime}\right\|_{0}$. On the other hand, $\left.\mathcal{F}_{+}(\psi)\right|_{\pi\left(\mathcal{A}_{\tau}(L)\right)}=\psi$ and $\left.\mathcal{F}_{+}\left(\psi^{\prime}\right)\right|_{\pi\left(\mathcal{A}_{\tau}(L)\right)}=\psi^{\prime}$ imply $\left\|\mathcal{F}_{+}(\psi)-\mathcal{F}_{+}\left(\psi^{\prime}\right)\right\|_{0} \geq\left\|\psi-\psi^{\prime}\right\|_{0}$.

\section{Separating sub-actions}

If $u \in C^{0}\left(\mathbb{T}^{d}\right)$ is a sub-action for a $C^{0}$ coercive Lagrangian, proposition 7.3 establishes that $\mathcal{A}_{\tau}(L) \subset \mathcal{N}_{\tau}(L, u)$. So it is natural to ask if there exists a sub-action whose nill locus is the smallest possible one, namely, it is equal to the Aubry set. We introduce then the following concept.

Definition 10.1. Let $L(x, v)$ be a $C^{0}$ coercive Lagrangian. We say that a subaction $u \in C^{0}\left(\mathbb{T}^{d}\right)$ is separating if $\mathcal{N}_{\tau}(L, u)=\mathcal{A}_{\tau}(L)$.

In weak KAM theory, global critical subsolutions of the Hamilton-Jacobi equation are analogous notions to separating sub-actions. Working with continuoustime, autonomous, strictly convex and superlinear $C^{2}$-Lagrangians on a smooth manifold without boundary, A. Fathi and A. Siconolfi (see [24]) proved the existence of $C^{1}$ critical subsolutions. Keeping the hypotheses on the Lagrangians but focusing on compact manifolds, P. Bernard showed in [8] not only the existence of $C^{1,1}$ critical subsolutions but also their density in the set of $C^{0}$ subsolutions for the uniform topology.

In a similar way, we prove in theorem 10.2 that separating sub-actions are quite typical in the discrete Aubry-Mather context. During the preparation of this paper, we become aware of a related study by M. Zavidovique [54] on separating subactions (or strict sub-solutions) in a general discrete setting given by cost functions defined on certain length spaces. We mention yet that the genericity of separating sub-actions has been proved in [32] in the context of ergodic optimization for symbolic dynamics.

Theorem 10.2. Let $L(x, v)$ be a $C^{0}$ coercive Lagrangian. Then, in the uniform topology, the subset of the continuous separating sub-actions is generic among all continuous sub-actions.

We will need some preliminary results. 
Lemma 10.3. Let $L(x, v)$ be a $C^{0}$ coercive Lagrangian. Then

$$
\operatorname{pr}^{1}\left(\bigcap_{u \text { is a sub-action }} \mathcal{N}_{\tau}(L, u)\right)=\bigcap_{u \text { is a sub-action }} \operatorname{pr}^{1}\left(\mathcal{N}_{\tau}(L, u)\right) .
$$

In other words, if $x \in \mathbb{R}^{d}$ and for any sub-action $u$ there exists $y \in \mathbb{R}^{d}$ such that $(x, y)$ is u-calibrated, then there exists $y \in \mathbb{R}^{d}$ such that $(x, y)$ is u-calibrated for any sub-action $u$.

Proof. The inclusion $\operatorname{pr}^{1}\left(\cap_{u} \mathcal{N}_{\tau}(L, u)\right) \subset \cap_{u} p^{1}\left(\mathcal{N}_{\tau}(L, u)\right)$ is obvious. Consider then $x \notin p r^{1}\left(\cap_{u} \mathcal{N}_{\tau}(L, u)\right)$. We want to show there exists a sub-action $u \in C^{0}\left(\mathbb{T}^{d}\right)$ such that $x \notin p r^{1}\left(\mathcal{N}_{\tau}(L, u)\right)$. Let $u_{0} \in C^{0}\left(\mathbb{T}^{d}\right)$ be a fixed sub-action. We know from corollary 6.4 that one can choose a constant $R_{\tau}>0$ such that $(x, v) \notin \mathcal{N}_{\tau}\left(L, u_{0}\right)$ whenever $\|v\|_{\infty}>R_{\tau}$. By hypothesis, for any $\|v\|_{\infty} \leq R_{\tau}$, there exist a subaction $u_{v} \in C^{0}\left(\mathbb{T}^{d}\right)$ and a constant $\eta_{v}>0$ satisfying $(x, w) \notin \mathcal{N}_{\tau}\left(L, u_{v}\right)$ whenever $\|v-w\|<\eta_{v}$. By extracting a finite subcover, one can find a finite collection of sub-actions $\left\{u_{1}, \ldots, u_{n}\right\} \subset C^{0}\left(\mathbb{T}^{d}\right)$, with $u_{k}=u_{v_{k}}$ for some $\left\|v_{k}\right\|_{\infty} \leq R_{\tau}$, such that $(x, v) \notin \bigcap_{k=1}^{n} \mathcal{N}_{\tau}\left(L, u_{k}\right)$ for any $\|v\|_{\infty} \leq R_{\tau}$.

Define thus $u:=\frac{1}{n+1} \sum_{k=0}^{n} u_{k} \in C^{0}\left(\mathbb{T}^{d}\right)$. Since the set of sub-actions is convex, $u$ turns out to be a sub-action. Besides, from $\mathcal{N}_{\tau}(L, u)=\bigcap_{k=0}^{n} \mathcal{N}_{\tau}\left(L, u_{k}\right)$, we immediately obtain $x \notin p r^{1}\left(\mathcal{N}_{\tau}(L, u)\right)$.

Lemma 10.4. Let $L(x, v)$ be a $C^{0}$ coercive Lagrangian. If $(x, v) \in \mathbb{T}^{d} \times \mathbb{R}^{d}$, then

$$
(x, v) \in \bigcap_{u \text { is a sub-action }} \mathcal{N}_{\tau}(L, u) \Longrightarrow x+\tau v \in \operatorname{pr}^{1}\left(\bigcap_{u \text { is a sub-action }} \mathcal{N}_{\tau}(L, u)\right) .
$$

In other words, if $(x, y) \in \mathbb{R}^{d} \times \mathbb{R}^{d}$ is u-calibrated for any sub-action $u$, then there exists $z \in \mathbb{R}^{d}$ such that $(y, z)$ is u-calibrated for any sub-action $u$.

Proof. Let us introduce a similar transform as in definition 9.7 by considering

$$
\tilde{\mathcal{F}}_{+}(u)(x):=\max _{y \in \mathbb{R}^{d}}\left[u(y)-S_{\tau}(x, y)\right], \quad \forall x \in \mathbb{R}^{d},
$$

where $u \in C^{0}\left(\mathbb{T}^{d}\right)$ is any sub-action. It is easy to see that $\tilde{\mathcal{F}}_{+}(u) \in C^{0}\left(\mathbb{T}^{d}\right)$ is again a sub-action satisfying $\tilde{\mathcal{F}}_{+}(u) \leq u$, with equality everywhere whenever $u$ behaves as a forward calibrated sub-action (see corollary 9.4 ).

We begin by proving

$$
\operatorname{pr}^{1}\left(\mathcal{N}_{\tau}(L, u)\right)=\left\{x \in \mathbb{T}^{d}: \tilde{\mathcal{F}}_{+}(u)(x)=u(x)\right\}, \quad \forall u \text { sub-action. }
$$

Indeed, if $(x, y)$ is $u$-calibrated, then $u(y)-u(x) \leq S_{\tau}(x, y) \leq \overline{\mathcal{L}}_{\tau}(x, y)=u(y)-u(x)$ which implies $\tilde{\mathcal{F}}_{+}(u)(x) \geq u(y)-S_{\tau}(x, y)=u(x)$ and therefore $\tilde{\mathcal{F}}_{+}(u)(x)=u(x)$. Conversely if $x \notin p r^{1}\left(\mathcal{N}_{\tau}(L, u)\right)$ then, by coerciveness of $L$ and periodicity of $u$, there exists $\eta>0$ such that $\overline{\mathcal{L}}_{\tau}(x, y) \geq u(y)-u(x)+\eta$ for any $y \in \mathbb{R}^{d}$. For any finite configuration $\left(x_{0}, x_{1}, \ldots, x_{n}\right)$ satisfying $x_{0}=x$, one has

$$
\begin{aligned}
\overline{\mathcal{L}}_{\tau}\left(x_{0}, x_{1}, \ldots, x_{n}\right) & =\overline{\mathcal{L}}_{\tau}\left(x_{0}, x_{1}\right)+\overline{\mathcal{L}}_{\tau}\left(x_{1}, \ldots, x_{n}\right) \\
& \geq\left[u\left(x_{1}\right)-u\left(x_{0}\right)+\eta\right]+\left[u\left(x_{n}\right)-u\left(x_{1}\right)\right] \geq u\left(x_{n}\right)-u\left(x_{0}\right)+\eta .
\end{aligned}
$$


By definition of $S_{\tau}(\underset{\tilde{\mathcal{F}}}{x}, y)$, one gets $S_{\tau}(x, y) \geq u(y)-u(x)+\eta$ for any $y \in \mathbb{R}^{d}$ or equivalently $u(x) \geq \tilde{\mathcal{F}}_{+}(u)(x)+\eta$.

We now prove the main induction step:

$$
\tilde{\mathcal{F}}_{+}(u)(x)=u(x) \text { and } \overline{\mathcal{L}}_{\tau}(x, y)=\tilde{\mathcal{F}}_{+}(u)(y)-\tilde{\mathcal{F}}_{+}(u)(x) \quad \Longrightarrow \quad \tilde{\mathcal{F}}_{+}(u)(y)=u(y) \text {. }
$$

Indeed, $u(y)-u(x) \leq S_{\tau}(x, y) \leq \overline{\mathcal{L}}_{\tau}(x, y)=\tilde{\mathcal{F}}_{+}(u)(y)-\tilde{\mathcal{F}}_{+}(u)(x)$, which implies first $u(y) \leq \tilde{\mathcal{F}}_{+}(u)(y)$ and therefore $u(y)=\tilde{\mathcal{F}}_{+}(u)(y)$.

We conclude the proof. If $(x, y)$ is $u$-calibrated for any sub-action $u$, on the one hand, $\tilde{\mathcal{F}}_{+}(u)(x)=u(x)$, on the other hand, since $(x, y)$ is also $\tilde{\mathcal{F}}_{+}(u)$-calibrated, $\tilde{\mathcal{F}}_{+}(u)(y)=u(y)$. We have proved that $y \in \cap_{u}\left\{\tilde{\mathcal{F}}_{+}(u)=u\right\}=p^{1}\left(\cap_{u} \mathcal{N}_{\tau}(L, u)\right)$ thanks to lemma 10.3 .

The following proposition gives another equivalent definition of the Aubry set.

Proposition 10.5. Let $L(x, v)$ be a $C^{0}$ coercive Lagrangian. Then

$$
\mathcal{A}_{\tau}(L)=\bigcap_{u \text { is a sub-action }} \mathcal{N}_{\tau}(L, u) .
$$

Proof. Let $(x, v) \in \cap_{u} \mathcal{N}_{\tau}(L, u)$. Lemma 10.4 shows there exists a configuration $\underline{x}=\left\{x_{k}\right\}_{k \geq 0}$ such that $\Pi_{\tau}(\underline{x})=(x, v)$ and $\left(x_{k}, x_{k+1}\right)$ is $u$-calibrated for any subaction $u$. Let us first show that

$$
l_{m}:=\overline{\mathcal{L}}_{\tau}\left(x_{0}, \ldots, x_{m}\right)+S_{\tau}\left(x_{m}, x_{0}\right) \rightarrow 0 \quad \text { when } \quad m \rightarrow+\infty .
$$

Since $\left\{\overline{\mathcal{L}}_{\tau}\left(x_{0}, \ldots, x_{m}\right)+S_{\tau}\left(x_{m}, x_{0}\right)\right\}_{m \geq 0}$ is uniformly bounded, one can choose a converging subsequence of $\left\{l_{m}\right\}$ and assume in addition that $\left\{x_{m}\left(\bmod \mathbb{Z}^{d}\right)\right\}$ converges to a point $x_{\infty} \in \mathbb{T}^{d}$. Define $u(x):=S_{\tau}\left(x_{\infty}, x\right)$, for all $x \in \mathbb{R}^{d}$. Proposition 8.3 shows that $u$ is a sub-action. By hypothesis of calibration on $\left\{x_{k}\right\}$, we have

$$
\overline{\mathcal{L}}_{\tau}\left(x_{k}, x_{k+1}\right)=S_{\tau}\left(x_{k}, x_{k+1}\right)=u\left(x_{k+1}\right)-u\left(x_{k}\right), \quad \forall k \geq 0 .
$$

More generally,

$$
\overline{\mathcal{L}}_{\tau}\left(x_{k}, x_{k+1}, \ldots, x_{m}\right)=S_{\tau}\left(x_{k}, x_{m}\right)=u\left(x_{m}\right)-u\left(x_{k}\right), \quad \forall m>k \geq 0 .
$$

By taking a subsequence of $\left\{x_{m}\right\}$, on obtains first $S_{\tau}\left(x_{k}, x_{\infty}\right)=u\left(x_{\infty}\right)-u\left(x_{k}\right)$, for all $k \geq 0$. By taking a subsequence of $\{k\}$, one obtains next

$$
u\left(x_{\infty}\right)=S_{\tau}\left(x_{\infty}, x_{\infty}\right)=0 \quad \text { and } \quad S_{\tau}\left(x_{k}, x_{\infty}\right)+S_{\tau}\left(x_{\infty}, x_{k}\right)=0, \quad \forall k \geq 0 .
$$

Notice that $x_{\infty}$ necessarily belongs to $\operatorname{pr}^{1}\left(\mathcal{A}_{\tau}(L)\right)$. Moreover,

$$
l_{m}=\overline{\mathcal{L}}_{\tau}\left(x_{0}, \ldots, x_{m}\right)+S_{\tau}\left(x_{m}, x_{0}\right)=S_{\tau}\left(x_{0}, x_{m}\right)+S_{\tau}\left(x_{m}, x_{0}\right), \quad \forall m \geq 0 .
$$

Letting $m \rightarrow+\infty$, one gets $l_{m} \rightarrow 0$ along a subsequence. We thus have shown that any accumulation point of $\left\{l_{m}\right\}$ is necessarily 0 .

Let us prove now that $(x, v) \in \mathcal{A}_{\tau}(L)$. By definition of $S_{\tau}$, there exist finite configurations $\left(x_{m}^{\epsilon}, x_{m+1}^{\epsilon}, \ldots, x_{n(m, \epsilon)}^{\epsilon}\right)$ such that $x_{m}^{\epsilon}=x_{m}, x_{n(m, \epsilon)}^{\epsilon}=x_{0}+p_{m}^{\epsilon}$ for some $p_{m}^{\epsilon} \in \mathbb{Z}^{d}$ and, for any $m$ fixed,

$$
\overline{\mathcal{L}}_{\tau}\left(x_{m}^{\epsilon}, x_{m+1}^{\epsilon}, \ldots, x_{n(m, \epsilon)}^{\epsilon}\right) \rightarrow S_{\tau}\left(x_{m}, x_{0}\right) \quad \text { when } \quad \epsilon \rightarrow 0 .
$$


We conclude that

$$
\begin{aligned}
\overline{\mathcal{L}}_{\tau}\left(x_{0}, \ldots, x_{m-1}, x_{m}^{\epsilon}, x_{m+1}^{\epsilon}, \ldots,\right. & \left.x_{n(m, \epsilon)}^{\epsilon}\right)= \\
& =l_{m}+\overline{\mathcal{L}}_{\tau}\left(x_{m}^{\epsilon}, x_{m+1}^{\epsilon}, \ldots, x_{n(m, \epsilon)}^{\epsilon}\right)-S_{\tau}\left(x_{m}, x_{0}\right)
\end{aligned}
$$

tends to 0 when $m$ is first chosen large enough and then $\epsilon$ is chosen close enough to 0 . Thus $(x, v) \in \mathcal{A}_{\tau}(L)$.

We now prove that separating sub-actions are generic among sub-actions.

Proof of theorem 10.2. Let $\left\{O_{n}\right\}_{n}$ be a countable family of open neighborhoods of the Aubry set such that $\cap_{n} O_{n}=\mathcal{A}_{\tau}(L)$. Let $\mathfrak{U}_{n}$ be the set of all $C^{0}\left(\mathbb{T}^{d}\right)$ sub-actions $u$ such that $\mathcal{N}_{\tau}(L, u) \subset O_{n}$. Since the subset of $C^{0}\left(\mathbb{T}^{d}\right)$ separating sub-actions is equal to $\cap_{n} \mathfrak{U}_{n}$, the statement of the theorem will be obtained if we show that, for the uniform topology, every $\mathfrak{U}_{n}$ is open and dense in the set of $C^{0}\left(\mathbb{T}^{d}\right)$ sub-actions.

Suppose on the contrary that $\mathfrak{U}_{n}$ is not open. So there exists a sequence of $C^{0}\left(\mathbb{T}^{d}\right)$ sub-actions $\left\{u_{k}\right\}_{k \geq 0}$ converging to some $u \in \mathfrak{U}_{n}$ and a sequence of points $\left\{\left(x_{k}, v_{k}\right)\right\}_{k \geq 0}$ such that, for all $k \geq 0,\left(x_{k}, v_{k}\right) \in \mathcal{N}_{\tau}\left(L, u_{k}\right)-O_{n}$. From corollary 6.4, we know there exists a positive constant $R_{\tau}$ such that $\left\|v_{k}\right\| \leq R_{\tau}$ for all $k$. By considering a suitable subsequence, we obtain a point $(x, v) \in \mathcal{N}_{\tau}(L, u)-O_{n}$ in contradiction with $\mathcal{N}_{\tau}(L, u) \subset O_{n}$.

Let us prove now that $\mathfrak{U}_{n}$ is dense. We first notice that, if $t \in(0,1), u \in \mathfrak{U}_{n}$ and $u^{\prime} \in C^{0}\left(\mathbb{T}^{d}\right)$ is any arbitrary sub-action, then

$$
\mathcal{N}_{\tau}\left(L, t u+(1-t) u^{\prime}\right)=\mathcal{N}_{\tau}(L, u) \cap \mathcal{N}_{\tau}\left(L, u^{\prime}\right) \subset O_{n}
$$

and therefore $t u+(1-t) u^{\prime} \in \mathfrak{U}_{n}$. In particular, in order to prove that $\mathfrak{U}_{n}$ is dense, it suffices to argue that $\mathfrak{U}_{n}$ is nonempty.

Corollary 6.4 assures that $(x, v) \notin \mathcal{N}_{\tau}(L, u)$ for any $\|v\|>R_{\tau}$ and any subaction $u$. Let $\mathbb{B}_{\tau}$ denote the closed ball of center $0 \in \mathbb{R}^{d}$ and radius $R_{\tau}$. Thanks to proposition 10.5 , for every point $(x, v) \in\left(\mathbb{T}^{d} \times \mathbb{B}_{\tau}\right)-O_{n}$, one can find a sub-action $u_{(x, v)} \in C^{0}\left(\mathbb{T}^{d}\right)$ and an open set $\mathcal{V}_{(x, v)} \subset \mathbb{T}^{d} \times \mathbb{R}^{d}$ containing $(x, v)$ such that

$$
(y, w) \notin \mathcal{N}_{\tau}\left(L, u_{(x, v)}\right), \quad \forall(y, w) \in \mathcal{V}_{(x, v)} .
$$

Hence, thanks to the compactness of $\left(\mathbb{T}^{d} \times \mathbb{B}_{\tau}\right)-O_{n}$, there exist a finite cover by open sets $\left\{\mathcal{V}_{1}, \ldots, \mathcal{V}_{m}\right\}$ of $\left(\mathbb{T}^{d} \times \mathbb{B}_{\tau}\right)-O_{n}$ and a finite collection of sub-actions $\left\{u_{1}, \ldots, u_{m}\right\} \subset C^{0}\left(\mathbb{T}^{d}\right)$, where $\mathcal{V}_{k}=\mathcal{V}_{\left(x_{k}, v_{k}\right)}$ and $u_{k}=u_{\left(x_{k}, v_{k}\right)}$ for some $\left(x_{k}, v_{k}\right)$, satisfying $\bigcap_{k=1}^{m} \mathcal{N}\left(L, u_{k}\right) \subset O_{n}$. Clearly $u:=\frac{1}{m} \sum_{k=1}^{m} u_{k} \in C^{0}\left(\mathbb{T}^{d}\right)$ belongs to $\mathfrak{U}_{n}$.

\section{Some results on rotation vectors}

We are interested in this section whether a minimizing configuration $\left\{x_{n}\right\}_{n \in \mathbb{Z}}$ in $\mathbb{R}^{d}$ is distributed according to a periodic pattern, that is, whether $\frac{1}{n}\left(x_{n}-x_{0}\right) \rightarrow \tau \omega$, where $\omega$ is some fixed vector in $\mathbb{R}^{d}$ called rotation vector. The case of monotone 
twist maps of the annulus (the one dimensional smooth ferromagnetic FrenkelKontorova model) has been completely solved by Aubry [3], Bangert [7] and Mather [49], [50] and [51]. One knows that a recurrent minimizing configuration admits a rotation number $\omega$ and belongs to the support of a minimizing measure of a Lagrangian of the form $L(x, v)-\langle\lambda, v\rangle$, with $\lambda$ and $\omega$ related by the Legendre relation $\omega=-\frac{\partial \bar{L}}{\partial \lambda}$, where $\bar{L}(\lambda)$ denotes the minimizing holonomic value of $L-$ $\lambda$. In particular, they proved that $\bar{L}(\lambda)$ is concave, decreasing and continuously differentiable with respect to $\lambda$. Moreover, $\bar{L}(\lambda)$ does not admit any open interval with irrational constant slope.

Our purpose in this section is to partially extend these results to the multidimensional case and to prove the existence of recurrent minimizing configurations with rotation vector as large in norm as we want.

Definition 11.1. We call rotation vector of a configuration $\left\{x_{k}\right\}_{k \in \mathbb{Z}}$ in $\mathbb{R}^{d}$ the limit (when it exists)

$$
\omega\left[\left\{x_{k}\right\}_{k \in \mathbb{Z}}\right]:=\frac{1}{\tau} \lim _{n-m \rightarrow+\infty} \frac{x_{n}-x_{m}}{n-m} .
$$

We call rotation vector of a holonomic measure $\mu \in \mathcal{P}_{\tau}\left(\mathbb{T}^{d} \times \mathbb{R}^{d}\right)$ with bounded support the vector

$$
\omega[\mu]:=\int_{\mathbb{T}^{d} \times \mathbb{R}^{d}} v d \mu(x, v) .
$$

Following Mather's terminology we introduce the so-called $\alpha$ and $\beta$ functions.

Definition 11.2. Let $L(x, v)$ be a $C^{0}$ superlinear Lagrangian. We call Mather's alpha function the opposite of the minimizing holonomic value of $L(x, v)-\langle\lambda, v\rangle$, $\lambda \in \mathbb{R}^{d}$,

$$
-\alpha_{L}(\tau, \lambda):=\bar{L}(\tau, \lambda)=\min \left\{\int(L(x, v)-\langle\lambda, v\rangle) d \mu(x, v): \mu \in \mathcal{P}_{\tau}\left(\mathbb{T}^{d} \times \mathbb{R}^{d}\right)\right\} .
$$

We call Mather's beta function the function

$$
\begin{array}{r}
\beta_{L}(\tau, \omega):=\inf \left\{\int L(x, v) d \mu(x, v): \mu \in \mathcal{P}_{\tau}\left(\mathbb{T}^{d} \times \mathbb{R}^{d}\right)\right. \text { has bounded support } \\
\text { and rotation vector } \left.\int v d \mu(x, v)=\omega\right\} .
\end{array}
$$

(We recall that $\mathcal{P}_{\tau}\left(\mathbb{T}^{d} \times \mathbb{R}^{d}\right)$ denotes the set of holonomic measures.)

We notice that, because of the superlinearity of $L, L-\lambda$ is again coercive (actually superlinear) and that $\bar{L}(\tau, \lambda)$ is indeed a minimum and not an infimum. We also point out that, in the definition of $\beta_{L}$, we prefer to restrict $\mu$ to have bounded support so that $\int v d \mu$ is well defined. We will show in a moment that the set where this infimum is taken is not empty and that the infimum is actually attained. Using standard convex analysis, we obtain the following proposition. 
Proposition 11.3. Let $L(x, v)$ be a $C^{0}$ superlinear Lagrangian. Then the two functions $\lambda \in \mathbb{R}^{d} \mapsto \alpha_{L}(\tau, \lambda) \in \mathbb{R}$ and $\omega \in \mathbb{R}^{d} \mapsto \beta_{L}(\tau, \omega) \in \mathbb{R}$ are convex superlinear obtained by Legendre transform:

$$
\alpha_{L}(\tau, \lambda)=\sup _{\omega \in \mathbb{R}^{d}}\left[\langle\lambda, \omega\rangle-\beta_{L}(\tau, \omega)\right] \quad \text { and } \quad \beta_{L}(\tau, \omega)=\sup _{\lambda \in \mathbb{R}^{d}}\left[\langle\lambda, \omega\rangle-\alpha_{L}(\tau, \lambda)\right] .
$$

In particular, both suprema are maxima.

The next lemma shows that Mather's beta function is well defined.

Lemma 11.4. For every $\omega \in \mathbb{R}^{d}$, there exists a holonomic measure $\mu$ such that

$$
\int v d \mu(x, v)=\omega \quad \text { and } \operatorname{supp}(\mu) \subset \mathbb{T}^{d} \times B_{\|\omega\|_{\infty}},
$$

where $B_{\|\omega\|_{\infty}}$ denotes the closed ball of center 0 and radius $\|\omega\|_{\infty}$.

Proof. If $\omega=p / q$, with $q \in \mathbb{Z}_{+}^{*}$ and $p \in \mathbb{Z}^{d}$, then clearly

$$
\mu_{p / q}:=\frac{1}{q} \sum_{k=0}^{q-1} \delta_{\left(\frac{k p}{q}, \frac{p}{\tau q}\right)}
$$

is a holonomic measure satisfying the statement of the lemma. For a general $\omega \in \mathbb{R}^{d}$, consider a sequence $\left\{p_{n} / q_{n}\right\}$, with $q_{n} \in \mathbb{Z}_{+}^{*}$ and $p_{n} \in \mathbb{Z}^{d}$, such that $\lim _{n \rightarrow \infty} p_{n} / q_{n}=$ $\omega$ and $\left\|p_{n} / q_{n}\right\|_{\infty} \leq\|\omega\|_{\infty}$. Let $\left\{\mu_{p_{n} / q_{n}}\right\}$ be the corresponding sequence of holonomic measures defined as above. Then this sequence is relatively compact for the narrow topology and any accumulation point $\mu_{\omega}$ is holonomic, $\mu_{\omega} \in \mathcal{P}_{\tau}\left(\mathbb{T}^{d} \times \mathbb{R}^{d}\right)$, and admits $\omega$ as a rotation vector.

We first recall a standard fact in convex analysis (see [14] for complete references).

Lemma 11.5. Let $f, g: \mathbb{R}^{d} \rightarrow \mathbb{R}$ be convex functions with full domain. Suppose that $f$ is the Legendre transform of $g$, namely,

$$
f(\lambda)=g^{*}(\lambda):=\sup \left\{\langle\lambda, \omega\rangle-g(\omega): \omega \in \mathbb{R}^{d}\right\}, \quad \forall \lambda \in \mathbb{R}^{d} .
$$

Then $f$ and $g$ are superlinear and $g$ is the Legendre transform of $f$. Moreover, for every $\lambda$ fixed (respectively $\omega$ fixed), the equation $f(\lambda)+g(\omega)=\langle\lambda, \omega\rangle$ admits at least one solution in $\omega$ (respectively in $\lambda$ ).

Proof of proposition 11.3. We show that $-\bar{L}(x, \lambda)$ is convex in $\lambda \in \mathbb{R}^{d}$. Indeed, for any $\lambda, \lambda^{*} \in \mathbb{R}^{d}$ and $t \in[0,1]$, if $\mu \in \mathcal{P}_{\tau}\left(\mathbb{T}^{d} \times \mathbb{R}^{d}\right)$ is a minimizing measure for $L_{t \lambda+(1+t) \lambda^{*}}$, then

$$
\begin{aligned}
\bar{L}\left(\tau, t \lambda+(1+t) \lambda^{*}\right) & =\int L_{t \lambda+(1+t) \lambda^{*}}(x, v) d \mu(x, v) \\
& =t \int L_{\lambda}(x, v) d \mu(x, v)+(1+t) \int L_{\lambda^{*}}(x, v) d \mu(x, v) \\
& \geq t \bar{L}(\tau, \lambda)+(1+t) \bar{L}\left(\tau, \lambda^{*}\right) .
\end{aligned}
$$


We now show that $-\bar{L}(\tau, \cdot)$ is the Legendre transform of $\beta_{L}(\tau, \cdot)$. Thanks to corollary 6.4 , we have

$$
-\bar{L}(\tau, \lambda)=\sup \left\{\int[\langle\lambda, v\rangle-L(x, v)] d \mu(x, v): \mu \in \mathcal{P}_{\tau}\left(\mathbb{T}^{d} \times \mathbb{R}^{d}\right),\right.
$$

and $\operatorname{supp}(\mu)$ is bounded $\}$.

Therefore, one can write

$$
\begin{aligned}
-\bar{L}(\tau, \lambda)=\sup _{\omega \in \mathbb{R}^{d}} \sup \left\{\langle\lambda, \omega\rangle-\int L(x, v) d \mu(x, v): \mu \in \mathcal{P}_{\tau}\left(\mathbb{T}^{d} \times \mathbb{R}^{d}\right),\right. \\
\left.\operatorname{supp}(\mu) \text { is bounded, and } \int v d \mu(x, v)=\omega\right\},
\end{aligned}
$$

namely, $-\bar{L}(\tau, \lambda)=\sup _{\omega \in \mathbb{R}^{d}}\left[\langle\lambda, \omega\rangle-\beta_{L}(\omega)\right]$. Proposition 11.3 follows then from lemma 11.5.

We are now able to prove the infimum is attained in the definition of $\beta_{L}(\tau, \omega)$.

Proposition 11.6. Let $L(x, v)$ be a $C^{0}$ superlinear Lagrangian. For every $\omega \in \mathbb{R}^{d}$, there exists a holonomic measure $\mu \in \mathcal{P}_{\tau}\left(\mathbb{T}^{d} \times \mathbb{R}^{d}\right)$ with bounded support such that

$$
\int_{\mathbb{T}^{d} \times \mathbb{R}^{d}} v d \mu(x, v)=\omega \quad \text { and } \quad \beta_{L}(\tau, \omega)=\int_{\mathbb{T}^{d} \times \mathbb{R}^{d}} L(x, v) d \mu(x, v) .
$$

Proof. We follow Mather's idea which says that the superlinearity of $L$ implies that, given a constant $C \in \mathbb{R}$, the set of Borel measures

$$
\left\{\|v\| \mu(d x, d v): \mu \in \mathcal{P}_{\tau}\left(\mathbb{T}^{d} \times \mathbb{R}^{d}\right), \text { and } \int L(x, v) d \mu(x, v) \leq C\right\}
$$

is tight. Let $\chi_{R}(x, v)$ be a test function taking its values in $[0,1]$ and satisfying $\chi(x, v)=1$ for all $\|v\| \leq R-1$ and $\chi_{R}(x, v)=0$ for all $\|v\| \geq R$. Let $\left\{\mu_{n}\right\}_{n \geq 0}$ be a sequence of Borel probability measures for which $\left\{\int L(x, v) d \mu_{n}(x, v)\right\}$ is uniformly bounded. So notice that, for every $\epsilon>0$ and $R$ sufficiently large, we have the inequality $\|v\|\left(1-\chi_{R}\right) \leq \epsilon(L(x, v)-\inf L)$, which clearly yields

$$
\lim _{R \rightarrow+\infty} \limsup _{n \rightarrow+\infty} \int\|v\|\left(1-\chi_{R}\right) d \mu_{n}(x, v)=0 .
$$

Suppose in addition that $\mu_{n}$ is holonomic,

$$
\lim _{n \rightarrow+\infty} \int L(x, v) d \mu_{n}(x, v)=\beta_{L}(\tau, \omega) \quad \text { and } \quad \int v d \mu_{n}(x, v)=\omega .
$$

We first extract a subsequence, that we again call $\left\{\mu_{n}\right\}_{n \geq 0}$, converging to a Borel measure $\mu$ in the sense that

$$
\begin{gathered}
\int f d \mu=\lim _{n \rightarrow+\infty} \int f d \mu_{n}, \quad \forall f \in C_{\text {compact }}^{0}\left(\mathbb{T}^{d} \times \mathbb{R}^{d}\right), \\
\int f d \mu \leq \liminf _{n \rightarrow+\infty} \int f d \mu_{n}, \quad \forall f \in C_{\text {bounded }}^{0}\left(\mathbb{T}^{d} \times \mathbb{R}^{d}\right), f \geq 0 .
\end{gathered}
$$


The tighness property actually implies for any $f \in C_{\text {bounded }}^{0}\left(\mathbb{T}^{d} \times \mathbb{R}^{d}\right)$

$$
\int f d \mu=\lim _{n \rightarrow+\infty} \int f d \mu_{n}, \quad \int f v d \mu=\lim _{n \rightarrow+\infty} \int f v d \mu_{n} .
$$

In particular, $\mu$ is a holonomic measure, it possesses a rotation vector $\omega$ and $\int L(x, v) d \mu(x, v) \leq \beta_{L}(\tau, \omega)$. However, as $\beta_{L}(\tau, \cdot)$ is the Legendre transform of $-\bar{L}(\tau, \cdot)$, there exists $\lambda_{\omega} \in \mathbb{R}^{d}$ such that $\beta_{L}(\tau, \omega)=\bar{L}\left(\tau, \lambda_{\omega}\right)+\left\langle\lambda_{\omega}, \omega\right\rangle$. We obtain

$$
\int\left(L(x, v)-\left\langle\lambda_{\omega}, v\right\rangle\right) d \mu(x, v)=\int L(x, v) d \mu(x, v)-\left\langle\lambda_{\omega}, \omega\right\rangle \leq \bar{L}\left(\tau, \lambda_{\omega}\right),
$$

which implies that $\mu$ is minimizing for $L_{\lambda_{\omega}}$ and, by corollary 6.4 , has bounded support.

Let us recall the definition of subdifferentials of a convex function. We restrict ourself to superlinear convex functions in order to avoid to pay attention to domains of definition.

Definition 11.7. Let $\alpha(\lambda)$ be a superlinear convex function with full domain. We call subdifferential of $\alpha$ at $\lambda^{*}$ the set

$$
\partial \alpha\left(\lambda^{*}\right):=\left\{\omega \in \mathbb{R}^{d}: \alpha(\lambda) \geq \alpha\left(\lambda^{*}\right)+\left\langle\omega, \lambda-\lambda^{*}\right\rangle, \quad \forall \lambda \in \mathbb{R}^{d}\right\} .
$$

A simple analysis of the definition of subdifferentials implies the following lemma.

Lemma 11.8. Let $\alpha(\lambda), \beta(\omega): \mathbb{R}^{d} \rightarrow \mathbb{R}$ be convex functions with full domain and obtained by Legendre transform one from the other. Then

$$
\lambda \in \partial \beta(\omega) \Longleftrightarrow \omega \in \partial \alpha(\lambda) \Longleftrightarrow \alpha(\lambda)+\beta(\omega)=\langle\lambda, \omega\rangle .
$$

In particular $\alpha(\lambda)$ is affine of slope $\omega$ on $\operatorname{int}(\partial \beta(\omega)$ ) (if not empty).

We summarize in the next proposition the properties of Mather's alpha and beta functions that are immediate consequences of convexity arguments.

Proposition 11.9. Let $L(x, v)$ be a $C^{0}$ superlinear Lagrangian.

i. Let $\omega, \lambda \in \mathbb{R}^{d}$ be related by Legendre relation $\lambda \in \partial \beta_{L}(\tau, \omega)$. Let $\mu$ be a holonomic measure of rotation vector $\omega$. Then $\mu$ realizes the minimum in the definition of $\beta_{L}(\tau, \omega)$ if, and only if, $\mu$ is minimizing for $L-\lambda$.

ii. Let $\lambda \in \mathbb{R}^{d}$. Then

$$
\partial \alpha_{L}(\tau, \lambda)=\{\omega[\mu]: \mu \text { is holonomic and minimizing for } L-\lambda\} .
$$

In particular, if $\bar{L}(\tau,$.$) is differentiable at \lambda$, then all minimizing measures of $L-\lambda$ have rotation vector $\omega[\mu]=\int v d \mu(x, v)=-\frac{\partial \bar{L}}{\partial \lambda}(\tau, \lambda)$.

iii. Let $\omega \in \mathbb{R}^{d}$ be such that $\operatorname{int}\left(\partial \beta_{L}(\tau, \omega)\right)$ is not empty. Then $\bar{L}(\tau,$.$) is affine$ of slope $\omega$ on $\operatorname{int}\left(\partial \beta_{L}(\tau, \omega)\right)$ and the Mather set of $L-\lambda$ is independent of $\lambda \in \operatorname{int}\left(\partial \beta_{L}(\tau, \omega)\right)$. 
Proof. Part $i$. Let $\lambda$ be a subdifferential of $\beta_{L}(\tau,$.$) at \omega$ and $\mu$ be a holonomic measure of rotation vector $\omega$. If $\mu$ realizes the minimum of $\beta_{L}(\tau, \omega)$, then

$$
\int[L(x, v)-\langle\lambda, v\rangle] d \mu(x, v)=\beta_{L}(\tau, \omega)-\langle\lambda, \omega\rangle=-\alpha_{L}(\tau, \lambda)=\bar{L}(\tau, \lambda),
$$

and $\mu$ is minimizing for $L-\lambda$. Conversely, if $\mu$ is minimizing for $L-\lambda$, then

$$
\begin{aligned}
\int L(x, v) d \mu(x, v) & =\int[L(x, v)-\langle\lambda, v\rangle] d \mu(x, v)+\langle\lambda, \omega\rangle \\
& =\bar{L}(\tau, \lambda)+\langle\lambda, \omega\rangle=-\alpha_{L}(\tau, \lambda)+\langle\lambda, \omega\rangle=\beta_{L}(\tau, \omega),
\end{aligned}
$$

and $\mu$ realizes the minimum of $\beta_{L}(\tau, \omega)$.

Part ii. Let $\omega$ be the rotation vector of a holonomic measure $\mu$ which is minimizing for $L-\lambda$. Then

$$
\int v d \mu(x, v)=\omega \quad \text { and } \quad \int[L(x, v)-\langle\lambda, v\rangle] d \mu(x, v)=\bar{L}(\tau, \lambda) .
$$

On the one hand, $\alpha_{L}(\tau, \lambda)+\beta_{L}(\tau, \omega) \geq\langle\lambda, \omega\rangle$ since $\alpha_{L}(\tau,$.$) is the Legendre trans-$ form of $\beta_{L}(\tau,$.$) . On the other hand,$

$$
\alpha_{L}(\tau, \lambda)+\beta_{L}(\tau, \omega) \leq-\bar{L}(\tau, \lambda)+\int L(x, v) d \mu(x, v)=\int\langle\lambda, v\rangle d \mu(x, v)=\langle\lambda, \omega\rangle
$$

We just have proved that $\alpha_{L}(\tau, \lambda)+\beta_{L}(\tau, \omega)=\langle\lambda, \omega\rangle$, that is, $\omega \in \partial \alpha_{L}(\tau, \lambda)$.

Conversely, suppose $\omega \in \partial \alpha_{L}(\tau, \lambda)$. Then $\lambda$ is a subdifferential of $\beta_{L}(\tau,$.$) at \omega$. Let $\mu$ be a holonomic probability measure of rotation $\omega$ realizing the minimum of $\beta_{L}(\tau, \omega)$. From part $i$, we know that $\mu$ is minimizing for $L-\lambda$.

Part iii. Let $\mu$ be a holonomic minimizing measure with respect to $L-\lambda$ for some $\lambda \in \operatorname{int}\left(\partial \beta_{L}(\tau, \omega)\right)$. Then $\bar{L}(\tau,$.$) is differentiable at \lambda$ and $\mu$ has rotation vector $\omega$. From part $i, \mu$ realizes the minimum in $\beta_{L}(\tau, \omega)$ and therefore is minimizing for $L-\lambda^{*}$ for any $\lambda^{*} \in \partial \beta_{L}(\tau, \omega)$.

An important issue in the multidimensional setting is to know whether or not any rotation vector is obtained by some minimizing configuration. In the ferromagnetic case, a simple application of ergodic theory shows that any $\omega=-\frac{\partial \bar{L}}{\partial \lambda}$, when $\lambda$ is a point of differentiability of $\bar{L}(\tau,$.$) , is obtained by generic recurrent calibrated$ minimizing configurations for $L-\lambda$. Proposition 11.13 gives a sligth extension of this fact. We follow Gomes' approach (see [34]) to show that actually all calibrated configurations of $L-\lambda$ have a rotation vector whenever $\bar{L}(\lambda)$ is differentiable at $\lambda$. Contrary to Gomes, we do not assume $L(x, v)$ to be ferromagnetic. We also prove the existence of minimizing configurations satisfying a stronger notion of rotation vector: for any $\lambda$ where $\bar{L}(\tau, \cdot)$ is differentiable in a neighborhood of $\lambda$ and has constant differential, any minimizing configuration of $L-\lambda$ stays at a bounded distance from a particular straight line. 
By standard convexity argument, the following directional differentials exist for all $\lambda, h \in \mathbb{R}^{d}$

$$
\begin{aligned}
\partial_{h}^{+} \bar{L}(\tau, \lambda) & :=\lim _{\rho \rightarrow 0^{+}} \frac{\bar{L}(\tau, \lambda+\rho h)-\bar{L}(\tau, \lambda)}{\rho}, \\
\partial_{h}^{-} \bar{L}(\tau, \lambda) & :=\lim _{\rho \rightarrow 0^{+}} \frac{\bar{L}(\tau, \lambda)-\bar{L}(\tau, \lambda-\rho h)}{\rho} .
\end{aligned}
$$

Theorem 11.10. Suppose $L(x, v)$ is $C^{0}$ and superlinear. Let $u_{\lambda} \in C^{0}\left(\mathbb{T}^{d}\right)$ be an arbitrary sub-action for $L-\lambda$. Then

i. For any $h \in \mathbb{R}^{d}$, for any $u_{\lambda}$-calibrated configuration $\left\{x_{k}\right\}_{k \in \mathbb{Z}}$, one has

$$
-\tau \partial_{h}^{-} \bar{L}(\tau, \lambda) \leq \liminf _{n-m \rightarrow \infty}\left\langle h, \frac{x_{n}-x_{m}}{n-m}\right\rangle \leq \limsup _{n-m \rightarrow \infty}\left\langle h, \frac{x_{n}-x_{m}}{n-m}\right\rangle \leq-\tau \partial_{h}^{+} \bar{L}(\tau, \lambda) .
$$

ii. If $\bar{L}(\tau, \cdot)$ is differentiable at $\lambda$, then any $u_{\lambda}$-calibrated configuration $\left\{x_{k}\right\}_{k \in \mathbb{Z}}$ has a rotation vector given by

$$
\omega\left[\left\{x_{k}\right\}_{k \in \mathbb{Z}}\right]=-\frac{\partial \bar{L}}{\partial \lambda}(\tau, \lambda)=\int v d \mu(x, v), \quad \forall \mu \in \mathcal{M}_{\tau}\left(L_{\lambda}\right) .
$$

There exist minimizing configurations for $L$ with rotation vector $\omega$ of arbitrarily large norm which are calibrated for $L-\lambda$, where $\omega=-\frac{\partial \bar{L}}{\partial \lambda}$.

iii. Let $\omega \in \mathbb{R}^{d}$ be given and $\Lambda_{\omega}:=\left\{\lambda \in \mathbb{R}^{d}:-\frac{\partial \bar{L}}{\partial \lambda}(\tau, \lambda)\right.$ exists and is equal to $\left.\omega\right\}$. If the interior of $\Lambda_{\omega}$ is not empty, for any $\lambda \in \operatorname{int}\left(\Lambda_{\omega}\right)$, for any $u_{\lambda}$-calibrated configuration $\left\{x_{k}\right\}_{k \in \mathbb{Z}}$ of $L-\lambda$, one has

$$
\sup _{k, n \in \mathbb{Z}}\left\|x_{k+n}-x_{k}-n \tau \omega\right\|_{\infty}<+\infty .
$$

We recall that a configuration is minimizing for $L$ if, and only if, it is minimizing for $L-\lambda$ for any $\lambda$. The previous theorem shows that the rotation vector of a minimizing configuration is in duality with the parameter $\lambda$ which let the configuration be calibrated by $L-\lambda$. In Mather theory, $\lambda$ is seen as a cohomology and $\omega$ as an homology. The parameter $\lambda$ in the standard one dimensional Frenkel-Kontorova model plays thus a special role.

Proof of theorem 11.10. Without loss of generality, we can suppose that $\bar{L}(\tau)=0$. Part $i$. Since $u_{\lambda}$ calibrates $\left\{x_{k}\right\}_{k \in \mathbb{Z}}$, one has

$$
u_{\lambda}\left(x_{n}\right)=u_{\lambda}\left(x_{m}\right)+\mathcal{L}_{\tau}\left(x_{m}, \ldots, x_{n}\right)-\left\langle\lambda, x_{n}-x_{m}\right\rangle-(n-m) \tau \bar{L}(\tau, \lambda), \quad \forall m<n .
$$

Let $\rho>0, h \in \mathbb{R}^{d}$ and $\lambda_{h}:=\lambda-\rho h$. If $u_{\lambda_{h}}$ is a continuous sub-action for $L-\lambda_{h}$,

$$
u_{\lambda_{h}}\left(x_{n}\right) \leq u_{\lambda_{h}}\left(x_{m}\right)+\mathcal{L}_{\tau}\left(x_{m}, \ldots, x_{n}\right)-\left\langle\lambda_{h}, x_{n}-x_{m}\right\rangle-(n-m) \tau \bar{L}\left(\tau, \lambda_{h}\right) .
$$

Therefore, by substracting the first equality to the above inequality, one obtains

$$
\tau \frac{\bar{L}(\tau, \lambda-\rho h)-\bar{L}(\tau, \lambda)}{\rho}-\frac{2}{\rho} \frac{\left\|u_{\lambda}-u_{\lambda_{h}}\right\|_{0}}{n-m} \leq\left\langle h, \frac{x_{n}-x_{m}}{n-m}\right\rangle,
$$


from which we immediately deduce

$$
-\tau \partial_{h}^{-} \bar{L}(\tau, \lambda)=\tau \lim _{\rho \rightarrow 0^{+}} \frac{\bar{L}(\tau, \lambda-\rho h)-\bar{L}(\tau, \lambda)}{\rho} \leq \liminf _{n-m \rightarrow \infty}\left\langle h, \frac{x_{n}-x_{m}}{n-m}\right\rangle .
$$

Replacing $h$ by $-h$, one thus gets

$$
\limsup _{n-m \rightarrow \infty}\left\langle h, \frac{x_{n}-x_{m}}{n-m}\right\rangle \leq \tau \lim _{\rho \rightarrow 0^{+}} \frac{\bar{L}(\tau, \lambda)-\bar{L}(\tau, \lambda+\rho h)}{\rho}=-\tau \partial_{h}^{+} \bar{L}(\tau, \lambda) .
$$

Part ii. If $\bar{L}(\tau, \cdot)$ is differentiable at $\lambda \in \mathbb{R}^{d}$, the previous inequalities become

$$
\left\langle\lim _{n-m \rightarrow \infty} \frac{x_{n}-x_{m}}{n-m}+\tau \frac{\partial \bar{L}}{\partial \lambda}(\tau, \lambda), h\right\rangle=0, \quad \forall h \in \mathbb{R}^{d} .
$$

We just have proved $\omega_{\lambda}:=\omega\left[\left\{x_{k}\right\}_{k \in \mathbb{Z}}\right]=-\frac{\partial \bar{L}}{\partial \lambda}(\tau, \lambda)$ exists. Notice yet that $\omega_{\lambda}$ satisfies the relation $\left\langle\lambda, \omega_{\lambda}\right\rangle=\beta_{L}\left(\tau, \omega_{\lambda}\right)-\bar{L}(\tau, \lambda)$. So if $\|\lambda\| \rightarrow+\infty$ among the set of points of differentiability of $-\bar{L}(\tau, \cdot)$, the superlinearity of $-\bar{L}(\tau, \cdot)$ implies $\left\|\omega_{\lambda}\right\| \rightarrow+\infty$

Part iii. Let $\lambda_{0} \in \operatorname{int}\left(\Lambda_{\omega}\right)$ and $\left\{x_{k}\right\}_{k \in \mathbb{Z}}$ be $u_{0}$-calibrated for some continuous subaction $u_{0}$ with respect to $L-\lambda_{0}$. Then, for any $k \in \mathbb{Z}$ and $n \in \mathbb{Z}_{+}$,

$$
\mathcal{L}_{\tau}\left(x_{k}, x_{k+1}, \ldots, x_{k+n}\right)=u_{0}\left(x_{k+n}\right)-u_{0}\left(x_{k}\right)+\left\langle\lambda_{0}, x_{k+n}-x_{k}\right\rangle+n \tau \bar{L}\left(\tau, \lambda_{0}\right) .
$$

Since $\bar{L}(\tau, \cdot)$ is affine on $\Lambda_{\omega}$, we have $\bar{L}(\tau, \lambda)-\bar{L}\left(\tau, \lambda_{0}\right)=-\left\langle\omega, \lambda-\lambda_{0}\right\rangle$, for any $\lambda \in \Lambda_{\omega}$. By taking any sub-action $u_{\lambda}$ of $L-\lambda$, one gets

$$
\begin{aligned}
& \mathcal{L}_{\tau}\left(x_{k}, \ldots, x_{k+n}\right)-\left\langle\lambda, x_{k+n}-x_{k}\right\rangle-n \tau \bar{L}(\tau, \lambda)-u_{\lambda}\left(x_{k+n}\right)+u_{\lambda}\left(x_{k}\right)= \\
& =\left\langle\lambda_{0}-\lambda, x_{k+n}-x_{k}-n \tau \omega\right\rangle+\left(u_{0}-u_{\lambda}\right)\left(x_{k+n}\right)-\left(u_{0}-u_{\lambda}\right)\left(x_{k}\right) .
\end{aligned}
$$

Since the left hand side of the previous equality is nonnegative, one finally gets

$$
\left\langle\lambda-\lambda_{0}, x_{k+n}-x_{k}-n \tau \omega\right\rangle \leq 4 \sup _{\left\|\lambda-\lambda_{0}\right\| \leq \epsilon}\left\|u_{\lambda}\right\|_{0}
$$

and $\left\|x_{k+n}-x_{k}-n \tau \omega\right\| \leq \frac{4}{\epsilon} \sup _{\left\|\lambda-\lambda_{0}\right\| \leq \epsilon}\left\|u_{\lambda}\right\|_{0}$.

We present an example where a calibrated configuration may not have a well defined rotation vector.

Example 11.11. Assume $d=1$ and $\tau=1$. Let $\ell: \mathbb{R} \times \mathbb{R} \rightarrow[0,1]$ be a $C^{\infty}$ function such that

$$
\ell^{-1}(1)=\{(0,0),(0,1 / 2),(1 / 2,0)\} \text { and } \ell^{-1}(0) \supset \mathbb{R}^{2}-(-1 / 4,3 / 4)^{2} .
$$

Define then $\mathcal{L}_{1}: \mathbb{R} \times \mathbb{R} \rightarrow \mathbb{R}_{+}$by

$$
\mathcal{L}_{1}(x, y)=1-\sum_{s \in \mathbb{Z}} \ell(x+s, y+s), \quad \forall x, y \in \mathbb{R} .
$$


Clearly, $\mathcal{L}_{1}$ is a $C^{\infty}$ function invariant by the diagonal action of $\mathbb{Z}$,

$$
\mathcal{L}_{1} \geq 0, \quad \mathcal{L}_{1}^{-1}(0)=S:=\bigcup_{s \in \mathbb{Z}}\{(s, s),(s, s+1 / 2),(s+1 / 2, s)\}
$$

and $\mathcal{L}_{1}>0$ everywhere on $\mathbb{R}^{2}-S$. If $\mathcal{L}_{2}(x, y)=|x-y|^{2}|x-y+1|^{2}|x-y-1|^{2}$, let us consider a nonnegative local interaction energy map given by

$$
\mathcal{L}(x, y)=\mathcal{L}_{1}(x, y) \mathcal{L}_{1}(x-1, y) \mathcal{L}_{1}(x, y-1)+\mathcal{L}_{2}(x, y), \quad \forall x, y \in \mathbb{R} .
$$

Notice that $\mathcal{L}$ is $C^{\infty}$, superlinear, invariant by the diagonal action of $\mathbb{Z}$,

$$
\mathcal{L} \geq 0 \quad \text { and } \quad \mathcal{L}^{-1}(0)=\bigcup_{s \in \mathbb{Z}}\{(s, s),(s, s+1),(s+1, s)\} .
$$

However, $\mathcal{L}$ does not satisfy a twist condition: there are points $\left(x_{0}, y_{0}\right) \in \mathbb{R}^{2}$ such that $\frac{\partial^{2} \mathcal{L}}{\partial x \partial y}\left(x_{0}, y_{0}\right)=0$. Indeed, since $\frac{\partial \mathcal{L}}{\partial y}(0,0)=0=\frac{\partial \mathcal{L}}{\partial y}(1,0)$, Rolle's theorem states that $\frac{\partial^{2} \mathcal{L}}{\partial x \partial y}\left(x_{0}, 0\right)=0$ for some $x_{0} \in(0,1)$.

Let then $L: \mathbb{T} \times \mathbb{R} \rightarrow \mathbb{R}$ denote the corresponding $C^{\infty}$ superlinear Lagrangian. We will exhibit a configuration $\left\{x_{k}\right\} u$-calibrated for any sub-action $u \in C^{0}(\mathbb{T})$ but without a well defined rotation vector. To that end, notice we have

$$
(x, v) \in \mathcal{A}_{1}(L) \Leftrightarrow \mathcal{L}(x, x+v)=0 \Leftrightarrow x=0(\bmod \mathbb{Z}) \text { and } v \in\{-1,0,1\} .
$$

So consider any sequence of positive integers $\left\{r_{i}\right\}_{i \geq 1}$ such that $\frac{1}{n} \sum_{i=1}^{n} r_{i}$ has at least two distinct accumulation points: $1 / \omega_{1}$ and $1 / \omega_{2}$. We define a configuration $\left\{x_{k}\right\}$ by

$$
x_{0}=0 \text { and } x_{k}=n \text { if } \sum_{i=1}^{n-1} r_{i}<|k| \leq \sum_{i=1}^{n} r_{i} .
$$

Notice that $\left(x_{k}(\bmod \mathbb{Z}), x_{k-1}-x_{k}\right) \in\{(0,-1),(0,0),(0,1)\}=\mathcal{A}_{1}(L)$. Therefore, proposition 7.3 guarantees $\left\{x_{k}\right\}$ is $u$-calibrated for any sub-action $u \in C^{0}(\mathbb{T})$. Nevertheless, the fact that

$$
\frac{n}{\sum_{i=1}^{n} r_{i}} \leq \frac{x_{k}}{k}<\frac{n}{\sum_{i=1}^{n-1} r_{i}} \text { whenever } \sum_{i=1}^{n-1} r_{i}<k \leq \sum_{i=1}^{n} r_{i}
$$

and the choice of the sequence $\left\{r_{i}\right\}$ imply that, when $k \rightarrow+\infty, x_{k} / k$ has $\omega_{1}$ and $\omega_{2}$ as accumulation points, which shows the configuration $\left\{x_{k}\right\}$ does not admit a rotation vector.

From now on we assume $L$ to be $C^{1}$, superlinear and ferromagnetic. We first notice that the set of critical configurations $\Gamma_{\tau}(L-\lambda)$ is independent from $\lambda$, that is, $\Gamma_{\tau}(L)=\Gamma_{\tau}(L-\lambda)$. A configuration calibrated for $L-\lambda$ is therefore critical for $L$. We also notice that $L-\lambda$ is ferromagnetic if, and only if, $L$ is ferromagnetic, and that the definition of the discrete-time Lagrangian dynamics $\left(\mathbb{T}^{d} \times \mathbb{R}^{d}, \Phi_{\tau}\right)$ is independent of $\lambda$ too.

According to theorem 7.7, Aubry sets are nonempty compact $\Phi_{\tau}$-invariant sets. Hence, as a consequence of theorem 11.10, the next result gives a sufficient condition for the existence of disjoint invariant sets with respect to the discrete-time Lagrangian dynamics. 
Proposition 11.12. Let $L(x, v)$ be a $C^{1}$ ferromagnetic superlinear Lagrangian. Suppose $\lambda, \lambda^{*} \in \mathbb{R}^{d}$ are points of differentiability of $\bar{L}(\tau, \cdot)$. Then

$$
\frac{\partial \bar{L}}{\partial \lambda}(\tau, \lambda) \neq \frac{\partial \bar{L}}{\partial \lambda}\left(\tau, \lambda^{*}\right) \quad \Longrightarrow \quad \mathcal{A}_{\tau}(L-\lambda) \cap \mathcal{A}_{\tau}\left(L-\lambda^{*}\right)=\emptyset .
$$

Proof. Suppose on the contrary $\left(x_{0}, v_{0}\right) \in \mathcal{A}_{\tau}(L-\lambda) \cap \mathcal{A}_{\tau}\left(L-\lambda^{*}\right)$. By the invariance of Aubry sets, we have

$$
\left(x_{k}, v_{k}\right):=\Phi_{\tau}^{k}\left(x_{0}, v_{0}\right) \in \mathcal{A}_{\tau}(L-\lambda) \cap \mathcal{A}_{\tau}\left(L-\lambda^{*}\right), \quad \forall k \in \mathbb{Z} .
$$

Define then $y_{0}=x_{0} \in[0,1)^{d}$ and recursively

$$
y_{k+1}=y_{k}+\tau v_{k} \in \mathbb{R}^{d} \text { for } k \geq 0 \text { and } y_{k-1}=y_{k}-\tau v_{k-1} \in \mathbb{R}^{d} \text { for } k \leq 0 .
$$

Let $u_{\lambda} \in C^{0}\left(\mathbb{T}^{d}\right)$ be a sub-action for $L-\lambda$ and let $u_{\lambda^{*}} \in C^{0}\left(\mathbb{T}^{d}\right)$ be a sub-action for $L-\lambda^{*}$. By proposition 7.3, the configuration $\left\{y_{k}\right\}_{k \in \mathbb{Z}}$ is simultaneously $u_{\lambda^{-}}$ calibrated and $u_{\lambda^{*} \text {-calibrated. Hence, theorem } 11.10 \text { implies }}$

$$
-\frac{\partial \bar{L}}{\partial \lambda}(\tau, \lambda)=\omega\left[\left\{y_{k}\right\}_{k \in \mathbb{Z}}\right]=-\frac{\partial \bar{L}}{\partial \lambda}\left(\tau, \lambda^{*}\right),
$$

which is a contradiction. Thus $\mathcal{A}_{\tau}(L-\lambda)$ and $\mathcal{A}_{\tau}\left(L-\lambda^{*}\right)$ are necessarily disjoint.

We have seen in theorem 11.10 that, if $\lambda$ is a point of differentiability of $\bar{L}(\tau, \cdot)$, $\omega=-\frac{\partial \bar{L}}{\partial \lambda}(\tau, \lambda)$ is the rotation vector of all configuration of $\mathcal{A}_{\tau}(L-\lambda)$. In the one dimensional case, $\bar{L}(\lambda)$ is differentiable everywhere and therefore all rotation numbers come from a minimizing configuration. We do not know whether this result persists in the multidimensional case. The following proposition is a step in that direction. We show that a vector $\omega$ which is extremal in $\partial \alpha_{L}(\tau, \lambda)$ for some $\lambda$ is the rotation vector of some minimizing configuration.

Proposition 11.13. Let $L(x, v)$ be a $C^{1}$ ferromagnetic superlinear Lagrangian. If $\omega$ is an extremal vector of $\partial \alpha_{L}(\tau, \lambda)$ for some $\lambda \in \mathbb{R}^{d}$, then it is the rotation vector of some minimizing configuration $\left\{x_{k}\right\}_{k \in \mathbb{Z}}$ calibrated for $L-\lambda$.

Proof. Let $\omega$ be an extremal point of $\partial \alpha_{L}(\tau, \lambda)$. By hypothesis, there exists a holonomic measure $\mu$ such that

$$
\int v d \mu(x, v)=\omega \quad \text { and } \quad \int[L(x, v)-\langle\lambda, v\rangle] d \mu(x, v)=\bar{L}(\tau, \lambda) .
$$

Theorem 6.10 guarantees that $\mu$ is $\Phi_{\tau}$-invariant. Furthermore, thanks to the extremal conditions on $\omega$ and on $\bar{L}(\tau, \lambda)$, we may assume that $\mu$ is $\Phi_{\tau}$-ergodic.

By the ergodicity of $\mu$, for almost all $(x, v) \in \mathbb{T}^{d} \times \mathbb{R}^{d}$, if $x_{0}$ is a representant of $x$ and $x_{n}=x_{0}+\tau \sum_{k=0}^{n-1} p r^{2} \circ \Phi_{\tau}(x, v)$, then

$$
\frac{1}{\tau} \lim _{n-m \rightarrow+\infty} \frac{x_{n}-x_{m}}{n-m}=\lim _{n-m \rightarrow+\infty} \sum_{k=m}^{n-1} p r^{2} \circ \Phi_{\tau}(x, v)=\int v d \mu(x, v)=\omega .
$$

Moreover, $\left\{x_{k}\right\}_{k \in \mathbb{Z}}$ is $u_{\lambda}$-calibrated for any sub-action $u_{\lambda}$ of $L-\lambda$ since

$$
\left(x_{k}\left(\bmod \mathbb{Z}^{d}\right), \frac{x_{k+1}-x_{k}}{\tau}\right)=\Phi_{\tau}^{k}(x, v) \in \mathcal{M}_{\tau}(L-\lambda) \subset \mathcal{N}_{\tau}\left(L-\lambda, u_{\lambda}\right) .
$$




\section{References}

[1] N. Anantharaman, On the zero-temperature or vanishing viscosity limit for certain Markov processes arising from Lagrangian dynamics, Journal of the European Mathematical Society 6 (2004), 207-276.

[2] G. Atkinson, Recurrence of co-cycles and random walks, The Journal of the London Mathematical Society 13 (1976), 486-488.

[3] S. Aubry and P. Y. Le Daeron, The discrete Frenkel-Kontorova model and its extensions: I. Exact results for the ground-states, Physica D 8 (1983), 381-422.

[4] S. Aubry, F. Axel and F. Vallet, Devil's staircases and Manhattan profile in an exact model for an incommensurate structure in an electric field, Journal of Physics C: Solid State Physics 18 (1985), 753-788.

[5] C. Baesens and R. S. MacKay, A novel preserved partial order for cooperative networks of units with overdamped second order dynamics, and application to tilted Frenkel-Kontorova chains, Nonlinearity 17 (2004), 567-580.

[6] C. Baesens and R. S. MacKay, Discommensuration theory and shadowing in FrenkelKontorova models, Physica D 216 (2006), 179-184.

[7] V. Bangert, Mather sets for twist maps and geodesics on tori, Dynamics Reported 1 (1988), $1-56$.

[8] P. Bernard, Existence of $C^{1,1}$ critical sub-solutions of the Hamilton-Jacobi equation on compact manifolds, Annales Scientifiques de l'École Normale Supérieure 40 (2007), 445-452.

[9] Th. Bousch, Un lemme de Mañé bilatéral, Comptes Rendus Mathématique 335 (2002), 533536.

[10] Th. Bousch, Le lemme de Mañé-Conze-Guivarc'h pour les systèmes amphidynamiques rectifiables, preprint, 2007.

[11] O. M. Braun and Y. S. Kivshar, The Frenkel-Kontorova model: concepts, methods, and applications, Texts and Monographs in Physics, Springer, Berlin, 2004.

[12] J. Brémont, Finite flowers and maximizing measures for generic Lipschitz functions on the circle, Nonlinearity 19 (2006), 813-828.

[13] X. Bressaud and A. Quas, Rate of approximation of minimizing measures, Nonlinearity 20 (2007), 845-853.

[14] P. Cannarsa and C. Sinestrari, Semiconcave functions, Hamilton-Jacobi equations, and optimal control, Progress in nonlinear differential equations and their applications 58, Birkhäuser, Boston, 2004.

[15] W. Chou and R. B. Griffiths, Ground states of one-dimensional systems using effective potentials, Physical Review B 34 (1986), 6219-6234.

[16] G. Contreras, Action potential and weak KAM solutions, Calculus of Variations and Partial Differential Equations 13 (2001), 427-458.

[17] G. Contreras and R. Iturriaga, Global minimizers of autonomous Lagrangians, $22^{\circ}$ Colóquio Brasileiro de Matemática, IMPA, Rio de Janeiro, 1999.

[18] G. Contreras, A. O. Lopes and Ph. Thieullen, Lyapunov minimizing measures for expanding maps of the circle, Ergodic Theory and Dynamical Systems 21 (2001), 1379-1409.

[19] L. C. Evans and D. A. Gomes, Linear programming interpretations of Mather's variational principle, ESAIM: Control, Optimisation and Calculus of Variations 8 (2002), 693-702.

[20] M. Falcone and M. Rorro, On a Variational Approximation of the Effective Hamiltonian, Proceedings of ENUMATH 2007, the 7th European Conference on Numerical Mathematics and Advanced Applications, Graz, Austria, September 2007, part 2, part 12 (2008), 719-726.

[21] A. Fathi, Théorème KAM faible et théorie de Mather sur les systèmes lagrangiens, Comptes Rendus des Séances de l'Académie des Sciences, Série I, Mathématique 324 (1997), 10431046. 
[22] A. Fathi, Solutions KAM faibles conjuguées et barrières de Peierls, Comptes Rendus des Séances de l'Académie des Sciences, Série I, Mathématique 325 (1997), 649-652.

[23] A. Fathi, The weak KAM theorem in Lagrangian dynamics, book to appear, Cambridge University Press.

[24] A. Fathi and A. Siconolfi, Existence of $C^{1}$ critical subsolutions of the Hamilton-Jacobi equation, Inventiones mathematicae 155 (2004), 363-388.

[25] L. M. Floría, C. Baesens and J. Gómez-Gardeñes, The Frenkel-Kontorova model, In: J. R. Chazottes and B. Fernandez (eds.), Dynamics of coupled map lattices and of related spatially extended systems, Lecture Notes in Physics 671, Springer, Berlin, 2005, pp. 209-240.

[26] G. Forni and J. N. Mather, Action minimizing orbits in Hamiltonian systems, In: S. Graffi (ed.), Transition to chaos in classical and quantum mechanics, Proceedings of a CIME summer school held in Montecatini Terme 1991, Lectures Notes in Mathematics 1589, Springer-Verlag, Berlin, 1994, pp. 92-186.

[27] N. Forcadel, C. Imbert and R. Monneau, Homogenization of fully overdamped FrenkelKontorova models, Journal of Differential Equations 246 (2009), 1057-1097.

[28] N. Forcadel, C. Imbert and R. Monneau, Homogenization of accelerated Frenkel-Kontorova models with $n$ types of particles, preprint, 2009.

[29] T. A. Frenkel and Ya. I. Kontorova, On the theory of plastic deformation and twinning I, II, III, Zhurnal Eksperimental'noi i Teoreticheskoi Fiziki 8 (1938) 89-95 (I), 1340-1349 (II), 1349-1359 (III).

[30] J. M. Gambaudo, P. Guiraud and S. Petite, Minimal configurations for the Frenkel-Kontorova model on a quasicrystal, Communications in Mathematical Physics 265 (2006), 165-188.

[31] E. Garibaldi and A. O. Lopes, On the Aubry-Mather theory for symbolic dynamics, Ergodic Theory and Dynamical Systems 28 (2008), 791-815.

[32] E. Garibaldi, A. O. Lopes and $\mathrm{Ph}$. Thieullen, On calibrated and separating sub-actions, Bulletin of the Brazilian Mathematical Society 40 (2009), 577-602.

[33] D. A. Gomes and A. M. Oberman, Computing the effective hamiltonian using a variational approach, SIAM J. Control Optim., 43, No. 3 (2004), 792-812.

[34] D. A. Gomes, Viscosity solution method and the discrete Aubry-Mather problem, Discrete and Continuous Dynamical Systems, Series A 13 (2005), 103-116.

[35] D. A. Gomes, A. O. Lopes and J. Mohr, The Mather measure and a large deviation principle for the entropy penalized method, preprint, 2007.

[36] R. M. Gray, Probability, random processes, and ergodic properties, Springer-Verlag, New York, 1988.

[37] R. B. Griffiths, H. J. Schellnhuber and H. Urbschat, Exact ground state of the FrenkelKontorova model with repeated parabolic potential. I. Basic results, Physical Review B 56 (1997), 8623-8630.

[38] M. R. Herman, Inégalités a priori pour des tores lagrangiens invariants par des difféomorphismes symplectiques, Publications Mathématiques de l'Institut des Hautes Études Scientifiques 70 (1989), 47-101.

[39] O. Jenkinson, Ergodic optimization, Discrete and Continuous Dynamical Systems 15 (2006), 197-224.

[40] O. Jenkinson and I. D. Morris, Lyapunov optimizing measures for $C^{1}$ expanding maps of the circle, Ergodic Theory and Dynamical Systems 28 (2008), 1849-1860.

[41] H. Koch, R. de la Llave and C. Radin, Aubry-Mather theory for functions on lattices, Discrete and Continuous Dynamical Systems 3 (1997), 135-151.

[42] R. Leplaideur, A dynamical proof for the convergence of Gibbs measures at temperature zero, Nonlinearity, 18 (2005), 2847-2880.

[43] R. de la Llave and E. Valdinoci, Ground states and critical points for generalized FrenkelKontorova models in $\mathbb{Z}^{d}$, Nonlinearity 20 (2007), 2409-2424. 
[44] R. Mañé, Generic properties and problems of minimizing measures of Lagrangian systems, Nonlinearity 9 (1996), 273-310.

[45] D. Massart, Stable norms of surfaces: local structure of the unit ball at rational directions, Geometric and Functional Analysis 7 (1997), 996-1010.

[46] D. Massart, On Aubry sets and Mather's action functional, Israel Journal of Mathematics 134 (2003), 157-171.

[47] D. Massart, Aubry sets vs Mather sets in two degrees of freedom, preprint, 2008.

[48] J. N. Mather, Existence of quasi-periodic orbits for twist homeomorphims of the annulus, Topology 21 (1982), 457-467.

[49] J. N. Mather, Minimal measures, Commentarii Mathematici Helvetici 64 (1989), 375-394.

[50] J. N. Mather, Differentiability of the minimal average action as a function of the rotation number, Boletim da Sociedade Brasileira de Matemática 21 (1990), 59-70.

[51] J. N. Mather, Action minimizing invariant measures for positive definite Lagragian systems, Mathematische Zeitschrift 207 (1991), 169-207.

[52] J. Moser, Monotone twist mappings and the calculus of variations, Ergodic Theory and Dynamical Systems 6 (1986), 401-413.

[53] M. Rorro, An approximation scheme for the effective Hamiltonian and application, Applied Numerical Mathematics, 56 (2006), 1238-1254.

[54] M. Zavidovique, Strict sub-solutions and Mañé potential in a discrete weak KAM theory, preprint, 2009. 